\title{
SUBSPACES WITH EQUAL CLOSURE
}

\author{
MARCEL DE JEU
}

Dedicated to Gabriëlle ten Have, Lianne, Sabine, and Rein de Graaf

1991 Mathematics Subject Classification. Primary 41A63 and 41-01; Secondary 41A10, 42A10, 44A60, 46F05, 46E10, 46E15, 46E20, 46E27, 46E30, 26E10, 22E30, 22E45.

Key words and phrases. Approximation, modules over polynomials and trigonometric functions in several variables, translation invariant subspaces, Bernstein problem, $L_{p}$-spaces, multidimensional moment problems, multidimensional quasi-analytic classes, quasi-analytic weights, harmonic analysis, Lie groups. 
ABSTRACT. We develop a method to approach the problem of describing the closure in topological vector spaces in analysis on $\mathbb{R}^{n}$ of a module over the polynomials or over the span of the exponentials with imaginary spectral parameter. The method is not restricted to a particular type of space or to cyclic modules. It is easy to apply and extends naturally to "mixed" versions. The results generalize and simplify several classical theorems and at the same time show that the conditions in some classical theorems are too stringent.

The fundamental observation is that it can under rather general conditions be shown that the closure of e.g. a module which is generated over the polynomials by a certain set, is equal to the closure of the module generated over the compactly supported smooth functions by this same set. The now translated problem of describing the closure of the second subspace is in general much better accessible than the original formulation.

We establish the method and give multidimensional non-cyclic applications in the Schwartz space, spaces of test functions and distributions, general spaces of Bernstein type and various spaces (including $L_{p}$-spaces) associated with a locally compact subset of $\mathbb{R}^{n}$. Cases which are already rather special still include e.g. dense translation invariant subspaces of the Schwartz space on $\mathbb{R}^{n}$, the classical sufficient criterion for the Bernstein problem on the real line, density of polynomials in $L_{p}$-spaces on subsets of $\mathbb{R}^{n}$ and as a consequence the determinacy of a class of multidimensional probability measures as it can be concluded directly from the measure.

The method shows that classical conditions on integrability or decay can often be relaxed, as e.g. with the density of polynomials in $L_{p}$-spaces and determinate measures. The reason for this is the use of quasi-analytic methods combined with our classification of multidimensional so-called quasi-analytic weights. One of the pertinent equivalences on the real line is that a weight (i.e. an arbitrary bounded non-negative function) $w$ on $\mathbb{R}$ is quasi-analytic, i.e. satisfies

$$
\sum_{m=1}^{\infty}\left\|x^{m} \cdot w(x)\right\|_{\infty}^{-1 / m}=\infty
$$

if and only if there exists a continuous even strictly positive weight $\widetilde{w}$ majorizing $w$, such that $s \mapsto-\log \widetilde{w}\left(e^{s}\right)$ is convex on $(-\infty, \infty)$ and such that

$$
\int_{0}^{\infty} \frac{\log \widetilde{w}(t)}{1+t^{2}}=-\infty
$$

Weights $\widetilde{w}$ as above are classical and they are apparently quasi-analytic weights themselves. Their good classical approximation properties can be said to be explained by this classification and the strong basic density results in this paper for arbitrary quasi-analytic weights.

We give an interpretation of the method in terms of Lie groups. In this interpretation the results in this paper (including many classical approximation results) are most naturally understood as situated on the unitary dual of $\mathbb{R}^{n}$, rather than on $\mathbb{R}^{n}$. The action of polynomials is then the action of the universal enveloping algebra of $\mathbb{R}^{n}$ on the smooth vectors in the various irreducible unitary representations of $\mathbb{R}^{n}$. This interpretation also indicates the possibility of generalization of the method to subsets of the unitary dual of certain classes of Lie groups. 


\section{Contents}

\begin{tabular}{lll}
\hline 1. Introduction and overview & 4
\end{tabular}

\begin{tabular}{|c|c|c|}
\hline Acknowledgments & 11
\end{tabular}

$\begin{array}{lll}2 . & \text { Notations and auxiliary results } & 11\end{array}$

3. Weights and associated spaces $\mathcal{O}_{w} \quad 13$

$\begin{array}{lll}3.1 . & \text { Definitions and preliminaries } & 13\end{array}$

$\begin{array}{lll}3.2 . & \text { Ostrowski's construction and weights on } \mathbb{R} & 16\end{array}$

\begin{tabular}{ll|l}
3.3. & Quasi-analytic weights & 17
\end{tabular}

$\begin{array}{lll}3.4 . & \text { Examples of quasi-analytic weights } & 26\end{array}$

\begin{tabular}{ll|l} 
3.5. Improving the spaces $\mathcal{O}_{w}$ & 27
\end{tabular}

\begin{tabular}{ll|l}
\hline 4. & Density in $\mathcal{O}_{w}$ & 28
\end{tabular}

\begin{tabular}{lll}
\hline 4.1. & Fourier transform on $\mathcal{O}_{w}^{\prime}$ & 29
\end{tabular}

4.2. Admissible spaces and their density in $\mathcal{O}_{w}$

$\begin{array}{lll}5 . \quad \text { Subspaces with equal closure } & 37\end{array}$

$\begin{array}{lll}\text { 5.1. } & \text { Formalization of the method } & 37\end{array}$

5.2. A partial filtration associated with types of weights 41

$\begin{array}{lll}6 . & \text { Applications } & 42\end{array}$

\begin{tabular}{|lll}
\hline 6.1. & The space $\mathcal{S}$ of rapidly decreasing functions & 42
\end{tabular}

6.2. The spaces $C_{c}^{m}(\Omega)$ and $C^{m}(\Omega)(0 \leq m \leq \infty)$ and their duals $\quad 44$

\begin{tabular}{lll}
\hline 6.3. & General spaces of Bernstein type & 47
\end{tabular}

$\begin{array}{lll}6.4 . \quad \text { Spaces associated with a locally compact set } X & 48\end{array}$

6.5. Determinate multidimensional measures 54

\begin{tabular}{lll}
\hline 7. & Interpretation in terms of Lie groups & 54
\end{tabular}

\begin{tabular}{ll|l}
7.1. & Formalism & 54
\end{tabular}

$\begin{array}{lll}7.2 . & \text { Perspectives } & 57\end{array}$

$\begin{array}{lll}7.3 . & \text { The } n \text {-torus } & 58\end{array}$

\begin{tabular}{|lll}
\hline Appendix A. Equal closure and continuous maps & 58
\end{tabular}

\begin{tabular}{lll}
\hline Appendix B. A Denjoy-Carleman type theorem in several variables & 59
\end{tabular}

References 60 


\section{INTRODUCTION AND OVERVIEW}

In this paper we develop a method to approach a certain class of approximation problems in an arbitrary number of variables. This class includes many instances of polynomial and trigonometric approximation as well as "mixed" versions, the meaning of which will be explained below. The present section is intended to motivate this method and contains an explanation and overview of the main ideas and results. The reader whose main interest lies in a possible application in a concrete situation is advised to read the present section, Definition 4.11 and Theorem 4.13 and then proceed to Sections 5 and 6 , reading other parts if and when necessary.

As to the motivation of the method, let us concentrate on polynomials for the moment and make some observations on the existing literature on polynomial approximation.

As a starting point, consider the following vector spaces and subspaces:

1. $C(K)$ - the continuous functions on a compact subset $K$ of $\mathbb{R}^{n}$-with the polynomials $\mathcal{P}$ in $n$ variables as subspace;

2. $C^{\infty}(\Omega)$ - the smooth functions on an open subset $\Omega$ of $\mathbb{R}^{n}$ —with $\mathcal{P}$ as subspace;

3. $C_{c}^{\infty}(\Omega)^{\prime}$ - the distributions on an open subset $\Omega$ of $\mathbb{R}^{n}$ —with $\mathcal{P}$ as subspace;

4. $L_{2}\left(\mathbb{R}^{n}, d x\right)$ with $\mathcal{P} \exp \left(-\|x\|^{2}\right)$ as subspace.

When the ambient spaces are supplied with the usual topologies (the weak topology in case of distributions), each indicated subspace is sequentially dense, as is asserted by classical theorems. Note that the subspaces are all modules over $\mathcal{P}$, even though the ambient spaces need not be. More precisely: the subspaces in question are cyclic modules over $\mathcal{P}$, generated as they are by either the constant 1 or the gaussian $\exp \left(-\|x\|^{2}\right)$. Evidently these classical density results can-although this is not the classical formulation - all be rephrased as ascertaining the density of certain cyclic modules over $\mathcal{P}$. It is not difficult to find more examples of this nature. Notwithstanding the formal similarity, the classical proofs of such results can differ substantially from case to case.

Furthermore, most results on the closure of a module over $\mathcal{P}$ are positive results for the density of the module under consideration. Methods of a general nature to quickly describe a (proper) closure do not appear to be known. As an example, the space $\mathcal{P} \cdot x \exp \left(-x^{2}\right)$ is not dense in the Schwartz space $\mathcal{S}(\mathbb{R})$ on the real line since any function in its closure vanishes at 0 . Its closure consists in fact precisely of all Schwartz functions vanishing at 0 , but a quick method yielding this description does not appear to be available.

Not much seems to be known about non-cyclic modules over $\mathcal{P}$. As a variation on the previous example, since the generators of $\mathcal{P} \cdot x \exp \left(-x^{2}\right)+\mathcal{P} \cdot(x-1) \exp \left(-x^{4}\right)$ do not have any common zero, there is now no obvious obstruction for this space to be dense in $\mathcal{S}(\mathbb{R})$. It is dense, but again an appropriate method to conclude this quickly appears to be absent.

In addition, we note that evidently much more is known in one variable than in several. The analysis in one variable is sometimes very refined, but this situation is not at all paralleled in higher dimensions.

Last but not least, one is tempted to say that the situations in which results are known about the closure of a module over $\mathcal{P}$ have the character of a list. If one encounters a situation which seems to be different from the known cases - e.g. the question whether $\mathcal{P} \cdot \exp \left(-\|x\|^{2}+\sin \sqrt{\|x\|^{2}+1}\right)$ is dense in $\mathcal{S}\left(\mathbb{R}^{n}\right)$, which it in 
fact is - then the existing literature is sometimes of little help. Of course existing proofs can often be adapted, but it may require some thought to actually do this in sufficient detail.

The above observations motivate the present paper. We present a method to approach the problem of describing the closure of modules over the polynomials in a large class of situations in topological vector spaces used in analysis on $\mathbb{R}^{n}$. This method, which extends naturally to trigonometric approximation and "mixed" versions (to be explained below), is uniform in nature and is valid in arbitrary dimension with, so it appears, quite some precision. It has a wide scope of application and it is easy to apply. We will establish the method in this paper, give some applications, propose an interpretation in terms of Lie groups and indicate the possibility of generalization from $\mathbb{R}^{n}$ to subsets of the unitary dual of certain classes of Lie groups.

Let us explain the method. The observation which lies at the basis of the approach is the following characterization of continuous maps.

Lemma 1.1. If $X$ and $Y$ are topological spaces, then a map $\phi: X \mapsto Y$ is continuous if and only if for all subsets $A$ and $B$ of $X$ having equal closure in $X$, the images $\phi(A)$ and $\phi(B)$ again have equal closure in $Y$.

Proof. If $\phi$ is continuous and $\bar{A}=\bar{B}$, then $\phi(A) \subset \phi(\bar{A})=\phi(\bar{B}) \subset \overline{\phi(B)}$, the latter inclusion holding by continuity. Hence $\overline{\phi(A)} \subset \overline{\phi(B)}$. By symmetry we conclude $\overline{\phi(A)}=\overline{\phi(B)}$. Conversely, let $\phi$ preserve the property of having equal closure. Since for all $A \subset X, \bar{A}$ and $A$ have equal closure in $X$, we have $\phi(\bar{A}) \subset \overline{\phi(\bar{A})}=\overline{\phi(A)}$ for all $A \subset X$, showing that $\phi$ is continuous.

With this result we will prove a uniform "translation" theorem along the following lines. We will establish topological vector spaces $\mathcal{O}_{w}$, depending on weights $w$ (to be discussed later on) and such that:

1. each $\mathcal{O}_{w}$ consists of smooth functions on $\mathbb{R}^{n}$;

2. $\mathcal{P}$ and the compactly supported smooth functions $\mathcal{D}$ are subspaces of each $\mathcal{O}_{w}$

3. $\overline{\mathcal{P}}=\overline{\mathcal{D}}$ in each $\mathcal{O}_{w}$;

4. it is common to have continuous maps from a space $\mathcal{O}_{w}$ to a topological vector space $V$ of the form $f \mapsto f \cdot g\left(f \in \mathcal{O}_{w}\right)$, where the dot indicates the appropriate type of action on a generator $g \in V$.

The weight $w$ under (4) has to be chosen judiciously, taking the properties of $g$ and the topology of $V$ into account. Such an appropriate choice need not always be possible, but in those cases where it is possible the previous lemma asserts that the property of having equal closure is preserved, i.e. $\overline{\mathcal{P} \cdot g}=\overline{\mathcal{D} \cdot g}$ in $V$. If $V$ is in addition locally convex and $\mathcal{G} \subset V$ is a set of generators to each of which the above principle applies, then

$$
\overline{\sum_{g \in \mathcal{G}} \mathcal{P} \cdot g}=\overline{\sum_{g \in \mathcal{G}} \mathcal{D} \cdot g}
$$

as a consequence of the Hahn Banach lemma.

The point is that the non-trivial analysis carried out for the spaces $\mathcal{O}_{w}$ is thus put to immediate use in other situations to validate a reformulation. Indeed, the problem of describing the closure of a module over $\mathcal{P}$ has, according to (1.1), now been translated to the apparently equivalent problem of describing the closure of 
the corresponding module over $\mathcal{D}$. The latter formulation of the problem is in general much better accessible, since the properties of $\mathcal{D}$ in relations to various spaces and their duals are usually well catalogued. As an example, it is immediate from the results in this paper that $\mathcal{P} \cdot \exp \left(-\sqrt{\|x\|^{2}+1} / \log \sqrt{\|x\|^{2}+2}\right)$ has the same closure in $L_{p}\left(\mathbb{R}^{n}, d x\right)(1 \leq p<\infty)$ as $\mathcal{D} \cdot \exp \left(-\sqrt{\|x\|^{2}+1} / \log \sqrt{\|x\|^{2}+2}\right)$. Since the latter space is simply $\mathcal{D}$, which is dense by general principles, the former space is therefore also dense. Such an immediate translation is valid in all examples so far and the reader may verify that this simplifies matters considerably, sometimes even reducing the situation to a triviality.

One can not expect to use the above method to prove that (1.1) holds in all possible situations, since this is simply not true. Examples where (1.1) is a meaningful but false statement are provided by some of the spaces of the classical continuous Bernstein problem, and by some $L_{2}$-spaces associated with non-determinate moment problems, both with $\mathcal{G}=\{1\}$. There are also situations (the two aforementioned classes of spaces again provide examples for this with $\mathcal{G}=\{1\}$ ), where (1.1) is meaningful and holds, but where our method does not allow us to conclude its validity. One could argue that these cases are subtle or at least more delicate than many practical situations one is likely to encounter, but this is admittedly a matter of taste. At any rate, we hope to convince the reader that the above circle of ideas has a rather large scope of application and that therefore, when used with appropriate caution, (1.1) can be regarded as a rule of thumb with theoretical foundation.

Returning to the "proof" of (1.1) we note that an extension presents itself, since the only relevant fact for its validity is that $\mathcal{P}$ is contained in the spaces $\mathcal{O}_{w}$ with the same closure as $\mathcal{D}$. Any other subspace with this property will do just as well. As it turns out, the span of $\exp i(\lambda, x)$ for $\lambda \in \mathbb{R}^{n}$ —we denote this span by $\mathcal{E}_{\text {im }}$-also has these properties together with $\mathcal{P}$; in fact the span of the exponentials corresponding to the spectral parameters in a somewhere dense subset of $\mathbb{R}^{n}$ (i.e. a subset such that its closure has non-empty interior) is already dense in $\mathcal{O}_{w}$. We thus obtain variations of (1.1) when replacing $\mathcal{P}$ by $\mathcal{E}_{\text {im }}$ (or such a proper subspace of $\mathcal{E}_{\text {im }}$ ), for all $g$ in a subset of $\mathcal{G}$ of our choice. These are the "mixed" versions of polynomial and trigonometric approximation alluded to above. One obtains a whole family of (in general) different subspaces, all with equal closure.

It is also possible, and as we will see in fact natural, to relax the conditions on the weight $w$ and develop versions of the $\mathcal{O}_{w}$ which contain $\mathcal{E}_{\text {im }}$ but not $\mathcal{P}$, still satisfying the crucial property $\overline{\mathcal{E}_{\mathrm{im}}}=\overline{\mathcal{D}}$. This evidently yields results on trigonometric approximation, valid in a wider setting than the polynomial context.

We will now discuss these spaces $\mathcal{O}_{w}$ and their subspaces. A moment's thought shows that if one aims at a large scope of application, then the spaces $\mathcal{O}_{w}$ should be small, implying that $\mathcal{P}$ and $\mathcal{D}$ should both be dense in the polynomial case and that $\mathcal{E}_{\text {im }}$ and $\mathcal{D}$ should both be dense in the trigonometric case. The spaces should consist of smooth functions to make the relevant maps well defined in as many cases as possible and finally the topology should be fairly strong to enhance the continuity of these maps.

It turns out that such spaces can already be found in the existing literature. Their potential use seems to have gone unnoticed. They have been introduced and studied in 33, 24], the latter reference aiming at simplifications of the former. We will analyze only a special case of these spaces, which is nevertheless sufficient to 
obtain significant extensions of the results in [loc.cit.] in the general situation (see Section 6.3).

The spaces $\mathcal{O}_{w}$ can be described as corresponding to the multidimensional differentiable analogue of the classical one-dimensional continuous Bernstein problem. For the sake of simplicity, let us restrict ourselves to the real line and assume that we are given a weight $w: \mathbb{R} \mapsto \mathbb{R}_{\geq 0}$. We do not need any regularity hypothesis on $w$, but we do assume that $w$ is bounded. The associated space $\mathcal{O}_{w}$ then consists of all $f \in C^{\infty}(\mathbb{R})$ such that $\lim _{|x| \rightarrow \infty} f^{(m)}(x) \cdot w(x)=0$ for $m=0,1,2, \ldots$. The seminorms $p_{m}$ defined by $p_{m}(f)=\left\|f^{(m)} \cdot w\right\|_{\infty}(m=0,1,2, \ldots)$ turn $\mathcal{O}_{w}$ into a locally convex (not necessarily Hausdorff) topological vector space. It is easy to show that $\mathcal{D}$ is dense in $\mathcal{O}_{w}$ for all bounded $w$. The subspace $\mathcal{E}_{\text {im }}$ is not considered in [33, 24], but we show that $\mathcal{E}_{\text {im }}$ is dense if $\lim _{|x| \rightarrow \infty} w(x)=0$, so that we then have $\overline{\mathcal{E}_{\mathrm{im}}}=\overline{\mathcal{D}}$ as required. If $w$ is rapidly decreasing then $\mathcal{P} \subset \mathcal{O}_{w}$ and one may ask what additional conditions on $w$ are sufficient to ensure that $\mathcal{P}$ is dense in $\mathcal{O}_{w}$, i.e. that $\overline{\mathcal{P}}=\overline{\mathcal{D}}\left(=\overline{\mathcal{E}_{\text {im }}}\right)$. It is known [33, 24] that this holds whenever $w$ is a so-called quasi-analytic weight, i.e. when

$$
\sum_{m=1}^{\infty}\left\|x^{m} \cdot w(x)\right\|_{\infty}^{-1 / m}=\infty .
$$

The term "quasi-analytic" refers to an application of the Denjoy-Carleman theorem in the proofs which is validated by this divergence.

We will (in arbitrary dimension) also prove this density of $\mathcal{P}$ in $\mathcal{O}_{w}$ for quasianalytic weights, but by an alternative approach which is more direct and brings out the natural connection between $\mathcal{P}$ and $\mathcal{E}_{\text {im }}$. This approach does not only yield an additional basic trigonometric approximation result (namely that the span of the exponentials corresponding to spectral parameters in a somewhere dense subset of $\mathbb{R}^{n}$ is already dense in $\mathcal{O}_{w}$ if $w$ is quasi-analytic), but at the same time shows what the natural interpretation in terms of Lie groups is, thus indicating how the setup can be generalized to certain classes of Lie groups.

Quasi-analytic weights are automatically rapidly decreasing. Although they have been studied in abstracto as above, examples of such weights appear to be sparse, if not absent. Weights on $\mathbb{R}$ of type $C \exp (-\epsilon|x|)$ and their minorants are quasianalytic, but for such weights this does not provide new insights since one can then prove the same - in fact even stronger - closure results by holomorphic methods rather than quasi-analytic ones.

In this paper we will not only give concrete non-trivial examples of quasi-analytic weights (Section 3.4) but we will actually classify them in arbitrary dimension. On the real line the equivalent characterization turns out to be familiar: a weight on $\mathbb{R}$ satisfies (1.2) if and only if there exists a continuous even strictly positive weight $\widetilde{w}$ majorizing $w$, such that $s \mapsto-\log \widetilde{w}\left(e^{s}\right)$ is convex on $(-\infty, \infty)$ and such that

$$
\int_{0}^{\infty} \frac{\log \widetilde{w}(t)}{1+t^{2}}=-\infty
$$

It has long been known that such weights $\widetilde{w}$ have good approximation properties in a number of situations, but it has not been noticed that they are essentially the same as the quasi-analytic weights in [23]. Furthermore, since in particular such a weight $\widetilde{w}$ is itself a quasi-analytic weight, the results in the present paper can be regarded as an explanation of these good classical approximation properties, in view 
of the possibility to push the strong results on equal closure in the corresponding space $\mathcal{O}_{\widetilde{w}}$ forward to a variety of other situations by Lemma 1.1.

This classification of quasi-analytic weights has another application. As the reader may verify from $(1.2)$, a probability measure $\mu$ on $\mathbb{R}$ is determinate by Carleman's theorem whenever there exists a strictly positive quasi-analytic weight $w$ such that $w^{-1} \in L_{1}(\mathbb{R}, \mu)$. Since the latter condition can be tested by an integral, the conclusion of determinacy can therefore be reached directly from the measure, which is evidently simpler than a computation of the moment sequence itself. A similar result for determinate measures holds in arbitrary dimension and in view of the explicit examples of quasi-analytic weights in Section 3.4 this result establishes a new class of multidimensional determinate measures. In Section 6.4 we will conclude the determinacy from a density result, but it is also possible to prove this determinacy in a more direct fashion as an application of an extended Carleman theorem in arbitrary dimension, using the classification of quasi-analytic weights on $\mathbb{R}^{n}$. We expect to report on this separately.

Returning to the spaces $\mathcal{O}_{w}$ we mention that the analogues of the closure results on the real line hold in arbitrary dimension. As to the proofs, the main idea for the proof of the density of $\mathcal{P}$ in $\mathcal{O}_{w}$ for quasi-analytic weights is standard, but the underlying technique involves Fourier analysis, quasi-analytic classes in several variables and vector valued integration in a combination which is perhaps new. It is interesting to note that, whereas most applications of the Fourier transform in approximation theory use its injectivity, we on the contrary use a result on its range. This fundamental role of harmonic analysis becomes more transparent when reformulating the method for Lie groups.

Turning to the applications, let us first make some comments of a general nature.

Let us assume that $V$ is a topological vector space, that $g \in V$ and that we wish to conclude that $\overline{\mathcal{P} \cdot g}=\overline{\mathcal{D} \cdot g}$ as above by finding a quasi-analytic weight $w$ such that the map $f \mapsto f \cdot g$ is continuous from $\mathcal{O}_{w}$ into $V$. The first requirement is obviously that $\mathcal{O}_{w} \cdot g \subset V$. Since $\mathcal{O}_{w}$ contains function which together with their derivatives grow at infinity at the rate of $w^{-1}$, this inclusion can roughly speaking be expected to hold when $g$ and its relevant derivatives decay at infinity at the same rate as $w$. In the same fashion, in case of trigonometric approximation one intuitively expects $\mathcal{O}_{w} \cdot g \subset V$ to hold for some weight $w$ tending to zero at infinity when $g$ tends to zero at infinity, together with its relevant derivatives. These imprecise statements are of course only useful as an intuitive orientation in practical situations. An interesting case occurs when $V$ is a $C^{\infty}\left(\mathbb{R}^{n}\right)$-module. Then $\mathcal{O}_{w} \cdot g \subset V$ for all $g \in V$ and all $w$ and chances increase that (1.1) holds for all generators.

Let us now consider the second requirement, i.e. the continuity of the map $f \mapsto$ $f \cdot g$ from $\mathcal{O}_{w}$ into $V$, assuming that it is well defined. Surprisingly enough one can prove in considerable generality that the hypothesis of continuity is not necessary to conclude (1.1). This is e.g. true for all $V$ that can be regarded as a subspace of a space of distributions, under mild conditions on the topology of $V$ which are satisfied by many common spaces. All spaces we have considered in this section are within the scope of this result. The underlying reason for the redundancy of the hypothesis of continuity is the fact that in this situation various spaces related to $V$ are Fréchet spaces; since it is in addition possible to "improve" the spaces $\mathcal{O}_{w}$ into Fréchet spaces, the closed graph theorem comes within range. 
This result on the redundancy of the hypothesis of continuity for a large class of spaces simplifies the application of our method. Indeed, the only hypothesis to be verified in all such situations is now an inclusion of the type $\mathcal{O}_{w} \cdot g \subset V$, which involves no more than the definition of $V$ as a set. The precise nature of $V$ and $g$ are to a large degree immaterial. For the detailed formulation of the general results in this direction we refer to Section 5 . Here we give the following example, which is a special case of Corollary 5.5.

Theorem 1.2. Let $X \subset \mathbb{R}^{n}$ be non-empty and let $V$ be an LF-space of functions on $X$ with a defining sequence $V^{1} \subset V^{2} \subset \ldots$ Suppose that convergence of a sequence in $V$ implies pointwise convergence on $X$ and that the spaces $V^{l}$ are all $C^{\infty}\left(\mathbb{R}^{n}\right)$-modules.

Let $\mathcal{G} \subset V$ be a non-empty set of generators and for $g \in \mathcal{G}$ let $L_{g}$ denote either $\mathcal{D}, \mathcal{P}$ or $\operatorname{Span}_{\mathbb{C}}\left\{\exp i(\lambda, x) \mid \lambda \in E_{g}\right\}$ where $E_{g}$ is a somewhere dense subset of $\mathbb{R}^{n}$ or, more generally, a uniqueness set for holomorphic functions on $\mathbb{C}^{n}$.

Then the closure in $V$ of the subspace $L=\sum_{g \in \mathcal{G}} L_{g} \cdot g$ is independent of the choice of the $L_{g}$.

The results of this type provide theoretical foundation for the idea of regarding (1.1) as a rule of thumb.

Closely related to the previous paragraphs and relevant for applications is the notion of an admissible space for an element $g$ in a topological vector space $V$, where we assume that $V$ is in the category of spaces for which the continuity hypothesis is redundant. Roughly speaking an admissible space for $g$ is any space $L$ of functions of a common nature for which there exists a weight $w$ such that $L$ is dense in $\mathcal{O}_{w}$ and such that $\mathcal{O}_{w} \cdot g \subset V$. The size of the collection of admissible spaces for $g$ determines the amount of freedom in varying the spaces acting on $g$ in (1.1). Stated intuitively (and disregarding the possibly relevant derivatives), the connection is that this freedom increases together with the maximum rate of growth of smooth functions $f$ such that $f \cdot g \in V$. If $V$ is a $C^{\infty}\left(\mathbb{R}^{n}\right)$-module then there is evidently maximal freedom for arbitrary $g \in V$ and one obtains results as Theorem 1.2, where the precise nature of $V$ is to a large degree irrelevant.

We will apply the method in various common spaces as they are encountered in analysis on $\mathbb{R}^{n}$. In all but a few examples we are able to give necessary and sufficient conditions for modules to be dense. The annihilator of non-dense modules can be described and in a number of cases this is sufficient to determine explicitly the closure of the module under consideration. This being said, let us indicate very briefly some of the additional features, leaving most of the material to Section 6 .

The first application is $\mathcal{S}$. We show that a closed translation invariant subspace of $\mathcal{S}$ is dense if and only if the Fourier transforms of its elements have no common zero. Consideration of mixed modules yields (under Fourier transform) multidimensional results of a type that has previously been considered on the real line by Mandelbrojt 21.

The second application treats various spaces of test functions and their duals. Classical results such as the sequential density of $\mathcal{P}=\mathcal{P} \cdot 1$ in $C_{c}^{\infty}(\Omega)^{\prime}$ are now an immediate consequence of the sequential density of $\mathcal{D}=\mathcal{D} \cdot 1$.

The third application is concerned with the general type of space for the differentiable multidimensional Bernstein problem. We extend the results in [33, 24]. A noteworthy particular case is the classical continuous Bernstein problem on the real line; the classical sufficient condition for the density of the polynomials in that 
situation is seen to be part of a more general picture as described in the theorem in this paper.

Our fourth application is concerned with various spaces associated with a locally compact subset $X$ of $\mathbb{R}^{n}$, including $L_{p^{-}}$-spaces and spaces of continuous functions. Specialization of these results yields e.g. the following: if $\mu$ is a Borel measure on $X$ (or its completion) such that $w^{-1} \in L_{1}(X, \mu)$ for a quasi-analytic weight $w$ which is strictly positive on $X$, then $\mathcal{P}$ is dense in $L_{p}(X, \mu)$ for all $1 \leq p<\infty$. The special case where $X=\mathbb{R}^{n}, p=2$ and $w(x)=\exp (-\epsilon\|x\|)$ for some $\epsilon>0$ is classical, but we now see that for general $X$ the polynomials are dense for all finite $p$ if $w^{-1} \in L_{1}(X, \mu)$ for a weight $w$ which is strictly positive on $X$ and for large $\|x\|$ equal to e.g. $\exp (-\epsilon\|x\| / \log a\|x\|)$ for some $\epsilon, a>0$. As alluded to above, for $X=\mathbb{R}^{n}$ this density in $L_{p^{-}}$spaces implies that such measures are determined by their moments. The resulting criterion for determinacy also extends the known case and is stated as our fifth and final application.

We also propose an interpretation of the spaces $\mathcal{O}_{w}$ in terms of Lie groups. In this interpretation, all the results under consideration in this paper are results situated on the unitary dual of $\mathbb{R}^{n}$. We will argue that the space $\mathcal{E}_{\text {im }}$ describes the action of the group algebra of $\mathbb{R}^{n}$ on the smooth vectors in the various irreducible unitary representations of $\mathbb{R}^{n}$. In this interpretation an element $x \in \mathbb{R}^{n}$ acts on the smooth vectors in the irreducible representation with spectral parameter $\lambda$ as multiplication by $\exp i(\lambda, x)$. Correspondingly, the function $\lambda \mapsto \exp i(\lambda, x)$ with $x \in \mathbb{R}^{n}$ fixed should at an abstract level be thought of as assigning to each irreducible unitary representation an endomorphism of its space of smooth vectors. Similarly, the action of an element of the universal enveloping algebra of $\mathbb{R}^{n}$ on all these spaces of smooth vectors is described globally on the unitary dual as multiplication by a polynomial, thus identifying $\mathcal{P}$. In this interpretation the canonical choice for the notation of the variable in many classical approximation results is actually $\lambda$ and not $x$. For further details we refer to Section 8, where we also indicate how the setup might be generalized to subsets of the unitary dual of certain classes of Lie groups. For the $n$-torus $\mathbb{T}^{n}$ this gives results on sequences.

This paper is organized as follows.

In Section 2 we establish the basic notations and recall some auxiliary results.

Section 3 is primarily concerned with a systematic study of weights, and notably quasi-analytic weights in several variables. The definition of these weights is shown to be related to frames in an essential way. We classify such weights and give explicit examples (including notably radial weights) in terms of elementary functions. We also show that e.g. quasi-analytic weights can often be assumed to be of class $C^{\infty}$, even though no regularity at all was imposed from the outset. Although this possibility of regularization is not essential for the remainder of the paper, it seems less than satisfactory to let it go unnoticed. This section also contains the definition of the spaces $\mathcal{O}_{w}$. An additional and important topic in this section is the "ubiquitous" possibility of finding spaces $\mathcal{O}_{w}$ that are Fréchet.

Section 1 contains the proof of the density results in $\mathcal{O}_{w}$. As was already mentioned above, there is - as far as the polynomials are concerned - an overlap of our results with [33, 24]. Since in [loc.cit.] $\mathcal{E}_{\text {im }}$ is not considered in its natural role and since our method of proof appears to be more direct than the reduction to the continuous case in one dimension as in [loc.cit.], and at the same time indicates what the possible generalization to Lie groups is, we feel that this section provides 
a more informative analysis of the spaces $\mathcal{O}_{w}$ than [loc.cit.]. This section also introduces the notion of admissible spaces for the various types of weights, i.e. the various subspaces of $\mathcal{O}_{w}$ of a common nature that are, depending on $w$, dense in $\mathcal{O}_{w}$ together with $\mathcal{D}$.

Section 5 may be regarded as the formalization of our method. It contains the general theorem on subspaces with equal closure, as well as three results on the redundancy of the hypothesis of continuity. The section concludes with the discussion of a partial filtration of topological vector spaces that can be related to the various types of weights. This leads naturally to the aforementioned notion of admissible spaces for elements of topological vector spaces. The partial filtration is also relevant for the regularity of associated Fourier transforms.

We treat the applications in Section 6 and conclude with the interpretation and possible generalizations to subsets of the unitary dual of certain classes of Lie groups in Section 17. This generalization is a feasible program for at least the unitary dual of $\mathbb{T}^{n}$, yielding results situated on $\mathbb{Z}^{n}$. We expect to report separately on this example.

In Appendix A we elaborate on Lemma 1.1, also considering the somewhat more delicate case of sequential closure in topological vector spaces.

Finally, in Appendix B we prove the theorem on quasi-analytic classes in several variables that lies at the heart of the density of $\mathcal{P}$ in $\mathcal{O}_{w}$ for a quasi-analytic weight $w$.

\section{ACKNOWLEDGMENTS}

It is a pleasure to thank Christian Berg, Jaap Korevaar and Jan Wiegerinck for helpful remarks.

\section{Notations AND AUXiliary RESUlts}

Throughout this paper we will be concerned with $\mathbb{R}^{n} ; n$ is fixed unless explicitly stated otherwise. We let $\left\{e_{1}, \ldots, e_{n}\right\}$ be the standard basis of $\mathbb{R}^{n}$. In case $n=1$ we write $e=e_{1}=1$. We supply $\mathbb{R}^{n}$ with the standard inner product $(.,$.$) and$ corresponding norm $\|\cdot\|$. The inner product is extended bilinearly to $\mathbb{C}^{n}$. The transpose of a linear transformation of $\mathbb{R}^{n}$ is defined with respect to this standard inner product.

$\mathcal{P}$ will denote the complex valued polynomials on $\mathbb{R}^{n} ; \mathcal{P}_{d}$ consists of the polynomials of degree at most $d$. If $\lambda \in \mathbb{C}$, then $e_{\lambda}: \mathbb{R}^{n} \mapsto \mathbb{C}$ is given by $e_{\lambda}(x)=\exp (\lambda, x)$ $\left(x \in \mathbb{R}^{n}\right)$. The complex span of the $e_{i \lambda}$ for $\lambda \in \mathbb{R}^{n}$ is denoted by $\mathcal{E}_{\mathrm{im}}$. We will refer to $\lambda$ as the spectral parameter for $e_{i \lambda}$, suppressing the factor $i$.

If $A \subset \mathbb{R}^{n}$ then $1_{A}$ denotes the characteristic (indicator) function of $A$.

All topological vector spaces in this paper are assumed to be complex, with the only and obvious exception of $\mathbb{R}^{n}$. The pairing between a topological vector space $V$ and its continuous dual $V^{\prime}$ is denoted as in $\left\langle v^{\prime}, v\right\rangle$. In our convention a topological vector space need not be Hausdorff. We mention explicitly that for a linear subspace $L$ of a locally convex space $V$, the closure $\bar{L}$ of $L$ can be described in the well known fashion as $\bar{L}=\left\{v \in V \mid\left\langle v^{\prime}, v\right\rangle=0 \forall v^{\prime} \in L^{\perp}\right\}$, where the annihilator $L^{\perp}$ is the subspace of $V^{\prime}$ consisting of all elements vanishing on $L$. For this to be valid $V$ need not be Hausdorff (see [30] or [1, p. II.45-46, p. II.64]). A Fréchet space is in our terminology a locally convex metrizable complete topological vector space. For LF-spaces, i.e. inductive limits of Fréchet spaces, we refer to [30, 14, 27. 
If $X$ is a topological space and $A \subset X$ then the sequential closure $\bar{A}^{s}$ is the set of all $x \in X$ for which there exists a sequence $\left\{a_{m}\right\}_{m=1}^{\infty} \subset A$ such that $\lim _{m \rightarrow \infty} a_{m}=$ $x$. Obviously $\bar{A}^{s} \subset \bar{A}$ and if all points in $X$ have a countable neighbourhood base then equality holds for all $A \subset X$. If $X$ is a topological vector space and $L$ is a subspace then $\bar{L}^{s}$ is evidently again a subspace.

If $\Omega$ is a non-empty subset of $\mathbb{R}^{n}$ and $m \in\{0,1,2, \ldots, \infty\}$, we let $C^{m}(\Omega)$ denote all functions of class $C^{m}$ on $\Omega$, endowed with the Fréchet topology of uniform convergence of all derivatives of order at most $m$ on compact subsets of $\Omega$. The elements in $C^{m}(\Omega)$ with compact support form a subspace $C_{c}^{m}(\Omega)$ which is endowed with the usual LF-topology. The dual spaces $C_{c}^{m}(\Omega)^{\prime}$ (resp. $\left.C^{m}(\Omega)^{\prime}\right)$ are identified with the subspaces of $C_{c}^{\infty}(\Omega)^{\prime}$ consisting of distributions of order at most $m$ (resp. the compactly supported distributions of order at most $m$ ), as a result of the density (in fact sequential density) of $C_{c}^{\infty}(\Omega)$ in $C_{c}^{m}(\Omega)$ (resp. the density of $C_{c}^{\infty}(\Omega)$ in $\left.C^{m}(\Omega)\right)$. Both $C_{c}^{m}(\Omega)^{\prime}$ and $C^{m}(\Omega)^{\prime}$ are modules over $C^{m}(\Omega)$. When $C_{c}^{m}(\Omega)^{\prime}$ and $C^{m}(\Omega)^{\prime}$ carry their weak topologies, $C_{c}^{\infty}(\Omega)$ is in both cases a sequentially dense subspace. We refer to 30, 14 for details. We write $\mathcal{D}$ for $C_{c}^{\infty}\left(\mathbb{R}^{n}\right)$ and $\mathcal{D}^{\prime}$ for the distributions on $\mathbb{R}^{n}$.

The space $\mathcal{S}$ of rapidly decreasing functions on $\mathbb{R}^{n}$ and its dual space of tempered distributions $\mathcal{S}^{\prime}$ are as usual. Any statement related to a topology on the spaces $C_{c}^{m}(\Omega), C^{m}(\Omega)$ or $\mathcal{S}$ always refers to the usual topologies as just described.

We adhere to the usual conventions concerning multi-indices. Thus, if $\alpha=$ $\left(\alpha_{1}, \ldots, \alpha_{n}\right) \in \mathbb{N}^{n}$, then $\partial^{\alpha}$ denotes the corresponding partial derivative of order $|\alpha|$. If necessary we will employ self-evident notations as $\partial_{x}^{\alpha}$ to indicate the variable on which the operator acts. For $x=\left(x_{1}, \ldots, x_{n}\right) \in \mathbb{C}^{n}$ and $\alpha \in \mathbb{N}^{n}$, we let $x^{\alpha}=x_{1}^{\alpha_{1}} \cdots x_{n}^{\alpha_{n}}$ and $|x|^{\alpha}=\left|x_{1}\right|^{\alpha_{1}} \ldots\left|x_{n}\right|^{\alpha_{n}}$.

We use the following normalization of the Fourier transform $\mathcal{F}$ :

$$
\mathcal{F}(f)=\widehat{f}=(2 \pi)^{-n / 2} \int_{\mathbb{R}^{n}} f(x) e^{-i(\lambda, x)} d x \quad\left(\lambda \in \mathbb{R}^{n}, f \in L_{1}\left(\mathbb{R}^{n}, d x\right)\right) .
$$

Its inverse is then

$$
\mathcal{F}^{-1}(f)=(2 \pi)^{-n / 2} \int_{\mathbb{R}^{n}} \mathcal{F}(f)(\lambda) e^{i(\lambda, x)} d x \quad\left(x \in \mathbb{R}^{n}, f, \mathcal{F}(f) \in L_{1}\left(\mathbb{R}^{n}, d x\right)\right) .
$$

$\mathcal{F}$ extends to tempered distributions by

$$
\langle\mathcal{F}(T), f\rangle=\langle T, \mathcal{F}(f)\rangle \quad\left(T \in \mathcal{S}^{\prime}, f \in \mathcal{S}\right)
$$

On occasion we will work in the extend non-negative real numbers $[0, \infty]$ with the usual conventions, including $0 \cdot \infty=0$ and $0^{0}=\infty^{0}=1$.

Let $R>0$. We will encounter non-increasing functions $w:[R, \infty) \mapsto \mathbb{R}_{>0}$ such that $s \mapsto-\log w\left(e^{s}\right)$ is convex on $[\log R, \infty)$. If $w \in C^{(2)}([R, \infty))$ then there exists $\rho \in C^{(1)}([R, \infty))$ with $\rho, \rho^{\prime} \geq 0$ on $[R, \infty)$ and such that

$$
w(t)=w(R) \exp \left(-\int_{R}^{t} \frac{\rho(s)}{s} d s\right) \quad(t \geq R)
$$

Conversely, if $\rho \in C^{(1)}([R, \infty))$ is non-negative and non-decreasing and a constant $w(R)>0$ is given, then (2.1) defines a function $w$ with the aforementioned properties. This type of equivalence is well known (cf. [22]). 


\section{Weights and associated spaces $\mathcal{O}_{w}$}

This section contains the definition of weights, the associated function spaces $\mathcal{O}_{w}$ and a first investigation of the relevant properties. The emphasis is on the classification of quasi-analytic weights. For these weights the criterion in Definition 3.2, which is suitable for applications of Denjoy-Carleman type theorems, can be shown to be related to the more practical (and at the same time familiar) criteria as in Theorem 3.14. This allows us to determine explicit examples of quasi-analytic weights in Section 3.4. Another main result is Theorem 3.22, where the ubiquity of complete spaces $\mathcal{O}_{w}$ for all types of weights under consideration is noted. A difference with most of the literature, apart from the arbitrary number of variables, is that we impose no regularity condition on weights.

3.1. Definitions and preliminaries. An arbitrary function $w: \mathbb{R}^{n} \mapsto \mathbb{R}_{\geq 0}$ is called a weight if it is bounded. The set of all weights is denoted by $\mathcal{W}$. The support $\sup w$ of a weight $w$ is the set $\left\{x \in \mathbb{R}^{n} \mid w(x) \neq 0\right\}$.

Given a weight $w$, we define the vector space $\mathcal{O}_{w}$ by

$$
\mathcal{O}_{w}=\left\{f \in C^{\infty}\left(\mathbb{R}^{n}\right) \mid \lim _{\|x\| \rightarrow \infty} \partial^{\alpha} f(x) \cdot w(x)=0 \forall \alpha \in \mathbb{N}^{n}\right\} .
$$

Then $\mathcal{O}_{w}$ is a locally convex topological vector space when supplied with the topology determined by the seminorms

$$
p_{\alpha}(f)=\left\|\partial^{\alpha} f \cdot w\right\|_{\infty} \quad\left(f \in \mathcal{O}_{w}, \alpha \in \mathbb{N}^{n}\right) .
$$

Remark 3.1. It is straightforward to check that $\mathcal{O}_{w}$ is Hausdorff if and only if the complement of the zero locus of $w$ is dense in $\mathbb{R}^{n}$. By the countability of the defining family of seminorms, this condition on the zero locus of $w$ is also the necessary and sufficient condition for metrizability of $\mathcal{O}_{w}$.

We note that if $w\left(x_{0}\right) \neq 0$ for some $x_{0} \in \mathbb{R}^{n}$, then convergence in $\mathcal{O}_{w}$ implies convergence of all derivatives at $x_{0}$. In the same vein, if $w$ is a weight such that $\inf _{x \in K} w(x)>0$ for every non-empty compact subset $K$ of $\mathbb{R}^{n}$, then $w$ is said to be separated from zero on compact sets. Convergence in the associated space $\mathcal{O}_{w}$ then implies uniform convergence of all derivatives on all compact sets, i.e. there is a continuous injection $\mathcal{O}_{w} \hookrightarrow C^{\infty}\left(\mathbb{R}^{n}\right)$.

We distinguish several classes of weights; their interrelations are summarized in Proposition 3.7. To start with, for $0 \leq d<\infty$ we let $\mathcal{W}_{d}$ denote the set of all weights $w$ such that $\lim _{\|x\| \rightarrow \infty}\|x\|^{d} w(x)=0$. The rapidly decreasing weights $\mathcal{W}_{\infty}$ are defined as $\mathcal{W}_{\infty}=\bigcap_{d=0}^{\infty} \mathcal{W}_{d}$; evidently $w \in \mathcal{W}_{\infty}$ if and only if $\mathcal{P} \subset \mathcal{O}_{w}$. If $w$ is a weight and if there exist $C \geq 0$ and $\epsilon>0$ such that $w(x) \leq C \exp (-\epsilon \| x \mid)$ for all $x \in \mathbb{R}^{n}$, then $w$ is a holomorphic weight. The nomenclature refers to the fact that the Fourier transform of an element of $\mathcal{O}_{w}^{\prime}$ is then a holomorphic function, as we will see in Section 4. The weight itself might be highly irregular. The set of holomorphic weights is denoted by $\mathcal{W}_{\text {hol }}$. For $w \in \mathcal{W}_{\text {hol }}$, define $0<\tau_{w} \leq \infty$ as the supremum of the set of all $\epsilon>0$ for which $w(x) \leq C_{\epsilon} \exp (-\epsilon\|x\|)$ for all $x$ and some $C_{\epsilon} \geq 0$. Then $\tau_{w}$ determines a tubular open neighbourhood of $\mathbb{R}^{n}$ in $\mathbb{C}^{n}$ for which the corresponding $e_{i \lambda}$ are still in $\mathcal{O}_{w}$.

We now come to the definition of the main class of weights that will concern us: the quasi-analytic weights. Here again the nomenclature refers to a property of Fourier transforms and not to the weight itself. The definition is as follows. 
With $v \in \mathbb{R}^{n}$ we associate $\tilde{v}: \mathbb{R}^{n} \mapsto \mathbb{R}$ defined by $\tilde{v}(x)=(v, x)$. If $w$ is a weight and $a \geq 0$, define the non-negative possibly infinite quantity

$$
M_{w}(v, a)=\left\||\tilde{v}|^{a} \cdot w\right\|_{\infty}
$$

Definition 3.2. If $\left\{v_{1}, \ldots, v_{n}\right\}$ is a basis of $\mathbb{R}^{n}$ such that

$$
\sum_{m=1}^{\infty} M_{w}\left(v_{j}, m\right)^{-1 / m}=\infty
$$

for all $1 \leq j \leq n$, then $w$ is called quasi-analytic with respect to $\left\{v_{1}, \ldots, v_{n}\right\}$. A weight is standard quasi-analytic if it is quasi-analytic with respect to the standard basis of $\mathbb{R}^{n}$. A weight is quasi-analytic if it is quasi-analytic with respect to some basis. The set of quasi-analytic weights is denoted by $\mathcal{W}_{q a}$.

We will see later on that $\mathcal{W}_{q a} \subset \mathcal{W}_{\infty}$. A small computation shows that holomorphic weights are quasi-analytic with respect to any basis. It is a non-trivial fact that a weight on $\mathbb{R}^{n}(n \geq 2)$ can be quasi-analytic with respect to one basis and not with respect to any other, apart from scaling of the original basis. The notion of quasi-analytic weights is therefore linked to frames in an essential way; we return to this point in Section 3.3 .

In one variable, a condition of type (3.1) already occurs in Bernstein's work on weighted polynomial approximation [1]. It is a recurring theme in the work of Nachbin (cf. 23), who in the case of one variable appears to have introduced the terminology. In several variables a proper subset of the quasi-analytic weights makes it appearance in [33, 24], although not under this name and not in the generality or central role in which we will study them.

We start with some elementary facts, which we state as lemmas for the ease of reference. The verification is left to the reader. The extended non-negative real numbers $[0, \infty]$ form the context in Lemma 3.4.

Lemma 3.3. 1. Let $\left\{a_{m}\right\}_{m=1}^{\infty} \subset \mathbb{R}_{>0}$ and suppose $\sum_{m=1}^{\infty} a_{m}^{-1}=\infty$. If $c \geq 0$, then $\sum_{m=1}^{\infty}\left(c+a_{m}\right)^{-1}=\infty$.

2. Let $\left\{a_{m}\right\}_{m=1}^{\infty} \subset \mathbb{R}_{\geq 0}$ be non-increasing. If $k$ and l are strictly positive integers, then $\sum_{m=1}^{\infty} a_{k m}=\infty$ if and only if $\sum_{m=1}^{\infty} a_{l m}=\infty$.

Lemma 3.4. Let $S$ be a non-empty set and suppose $g, w: S \mapsto \mathbb{R}_{\geq 0}$.

1. If $a, b \geq 0$ then $\left\|g^{\lambda a+(1-\lambda) b} \cdot w\right\|_{\infty} \leq\left\|g^{a} \cdot w\right\|_{\infty}^{\lambda}\left\|g^{b} \cdot w\right\|_{\infty}^{1-\lambda}$ for $0 \leq \lambda \leq 1$.

2. If $\|w\|_{\infty}<\infty$ and $\left\|g^{a} \cdot w\right\|_{\infty}=\infty$ for some $a>0$, then $\left\|g^{b} \cdot w\right\|_{\infty}=\infty$ for all $b \geq a$.

3. If $\|w\|_{\infty}=1$, then $\left\|g^{a} \cdot w\right\|_{\infty}^{1 / a} \leq\left\|g^{b} \cdot w\right\|_{\infty}^{1 / b}$ whenever $0<a \leq b$.

Remark 3.5. The first part of Lemma 3.4 implies that $\left\{M_{w}(v, m)\right\}_{m=0}^{\infty}$ is an (obviously non-negative) logarithmically convex sequence for all weights $w$ and all $v \in \mathbb{R}^{n}$. We will use this repeatedly.

Given the fact that a weight can be quasi-analytic with respect to essentially one basis, it is natural to consider the action of the general linear group. For our purposes it is actually better to consider the action of the affine group Aff, where the action on functions is defined by $A f(x)=f\left(A^{-1} x\right)$ for $f: \mathbb{R}^{n} \mapsto \mathbb{C}$ and $A \in$ Aff. 
Lemma 3.6. Let $w$ be a quasi-analytic weight with respect to $\left\{v_{1}, \ldots, v_{n}\right\}$. Then $w$ is rapidly decreasing. If $A_{0} \in \mathrm{Aff}$ is linear, then $A_{0} w$ is quasi-analytic with respect to $\left\{\left(A_{0}^{-1}\right)^{t} v_{1}, \ldots,\left(A_{0}^{-1}\right)^{t} v_{n}\right\}$. If $T \in \mathrm{Aff}$ is a translation, then $T w$ is quasi-analytic with respect to $\left\{v_{1}, \ldots, v_{n}\right\}$.

Proof. To see that $w$ is rapidly decreasing, first note that - in view of (3.1) - the second part of Lemma 3.4 implies that $\left\|\tilde{v}_{j}^{m} \cdot w\right\|_{\infty}<\infty$ for all $1 \leq j \leq n$ and all $m \geq 1$. Trivially this also holds when $m=0$. Next choose a disjoint decomposition $\mathbb{R}^{n}=\bigcup_{j=1}^{n} S_{j}$ such that for $j=1 \ldots, n$ we have $\max _{i=1, \ldots, n}\left|\tilde{v}_{i}(x)\right|=\left|\tilde{v}_{j}(x)\right|$ for all $x \in S_{j}$. Then for $\alpha \in \mathbb{N}^{n}$ we have

$$
\left\|\prod_{i=1}^{n} \tilde{v}_{i}^{\alpha_{i}} \cdot w\right\|_{\infty} \leq \sum_{j=1}^{n}\left\|1_{S_{j}} \prod_{i=1}^{n} \tilde{v}_{i}^{\alpha_{i}} \cdot w\right\|_{\infty} \leq \sum_{j=1}^{n}\left\|\tilde{v}_{j}^{|\alpha|} \cdot w\right\|_{\infty}<\infty,
$$

by the finiteness noted above. Hence $w$ is rapidly decreasing.

The statement concerning the action of $A_{0}$ is immediate from the definitions.

Turning to the translation part, let $T=T_{y}$ where $T_{y}$ is the translation $T_{y}(x)=$ $x+y$ over $y$. We may assume $\|w\|_{\infty}=1$. Using the third part of Lemma 3.4 it then follows readily that $\left\|\tilde{v}_{j}^{m} \cdot T_{y} w\right\|_{\infty}^{-1 / m} \geq\left(\left|\left(v_{j}, y\right)\right|+\left\|\tilde{v}_{j}^{m} \cdot w\right\|_{\infty}^{1 / m}\right)^{-1}$ for all $m \geq 1$. If $\left\|\tilde{v}_{j}^{m} \cdot w\right\|_{\infty}=0$ for some $m \geq 1$, then $\left\|\tilde{v}_{j}^{m} \cdot w\right\|_{\infty}=0$ for all $m \geq 1$ and it follows trivially from the estimate that $\sum_{m=1}^{\infty} M_{T_{y} w}\left(v_{j}, m\right)^{-1 / m}=\infty$. In case $\left\|\tilde{v}_{j}^{m} \cdot w\right\|_{\infty} \neq 0$ for all $m \geq 1$, the necessary divergence is implied by the estimate and the first part of Lemma 3.3 .

Proposition 3.7. We have $\mathcal{W}_{\text {hol }} \subset \mathcal{W}_{\text {qa }} \subset \mathcal{W}_{\infty} \subset \mathcal{W}_{d} \subset \mathcal{W}_{d^{\prime}} \subset \mathcal{W}_{0} \subset \mathcal{W}$ for $d \geq d^{\prime} \geq 0$. Each of the sets of weights in this chain of inclusions is a positive convex cone, with the exception of $\mathcal{W}_{q a}$ which is closed under multiplication by non-negative scalars, but not under addition.

The affine group Aff leaves $\mathcal{W}_{\text {hol }}, \mathcal{W}_{\text {qa }}, \mathcal{W}_{d}(0 \leq d \leq \infty), \mathcal{W}, \mathcal{D}, \mathcal{S}, \mathcal{E}_{\text {im }}$ and $\mathcal{P}$ invariant. The orbit of a quasi-analytic weight under the general linear group contains a standard quasi-analytic weight. If $A \in \mathrm{Aff}$, then $f \mapsto A f$ implements an isomorphism between the topological vector spaces $\mathcal{O}_{w}$ and $\mathcal{O}_{A w}$.

Proof. The fact that $\mathcal{W}_{q a}$ is not closed under addition will be illustrated in the discussion following Proposition 3.10. It has already been noted that $\mathcal{W}_{\text {hol }} \subset \mathcal{W}_{q a}$ and the rest is either a routine verification or contained in Lemma 3.6.

Remark 3.8. Let us note explicitly that $\mathcal{D}, \mathcal{S}, \mathcal{E}_{\text {im }}$ and $\mathcal{P}$ are subspaces of $\mathcal{O}_{w}$ for all $w \in \mathcal{W}_{q a}$ and that Proposition 3.7 implies that in order to prove that these subspaces are dense in $\mathcal{O}_{w}$ for all $w \in \mathcal{W}_{q a}$, it is sufficient to do so for standard quasi-analytic weights.

The definition of the various classes of weights involves only the behaviour at infinity; this is a special case of the following lemma.

Proposition 3.9. Let $w$ and $\widetilde{w}$ be weights and suppose there exist $C \geq 0$ and $R \geq 0$ such that $w(x) \leq C \widetilde{w}(x)$ for all $x$ with $\|x\| \geq R$. Then, if $\widetilde{w} \in \mathcal{W}_{d}$ for some $0 \leq d \leq \infty$ (resp. $\widetilde{w} \in \mathcal{W}_{\text {hol }}$ ), we also have $w \in \mathcal{W}_{d}$ (resp. $w \in \mathcal{W}_{\text {hol }}$ and $\tau_{w} \geq \tau_{\widetilde{w}}$ ). If $\widetilde{w}$ is quasi-analytic with respect to the basis $\left\{v_{1}, \ldots, v_{n}\right\}$, then so is $w$. 
Proof. This is immediate from the definitions, except when $w \in \mathcal{W}_{q a}$. In that case Lemma 3.6 allows us to assume that $w$ is standard quasi-analytic. Fix $1 \leq j \leq n$. If there exists $r \geq 0$ such that $w(x)=0$ whenever $\left|x_{j}\right| \geq r$, then $M_{w}\left(e_{j}, m\right) \leq$ $r^{m}\|w\|_{\infty}$ for all $m$, making it obvious that $\sum_{m=1}^{\infty} M_{w}\left(e_{j}, m\right)^{-1 / m}=\infty$. If $w$ does not have such bounded support in the direction of the $j$-th coordinate, let $C$ and $R$ be as in the hypotheses of the proposition. Choose and fix $y \in \mathbb{R}^{n}$ with $\left|y_{j}\right| \geq R+1$ and $w(y) \neq 0$. A comparison of geometric progressions shows that there exists $N$ with the property that for all $m \geq N$ and for all $x \in \mathbb{R}^{n}$ such that $\left|x_{j}\right| \leq R$ we have

$$
\left|x_{j}^{m} \cdot w(x)\right| \leq\left|y_{j}^{m} \cdot w(y)\right| .
$$

Then the necessary divergence follows from the observation that for $m \geq N$ :

$$
\begin{aligned}
M_{w}\left(e_{j}, m\right) & =\sup \left\{\left|x_{j}^{m} \cdot w(x)\right| \mid x \in \mathbb{R}^{n}\right\} \\
& =\sup \left\{\left|x_{j}^{m} \cdot w(x)\right|\left|x \in \mathbb{R}^{n},\right| x_{j} \mid \geq R\right\} \\
& \leq \sup \left\{\left|x_{j}^{m} \cdot w(x)\right| \mid x \in \mathbb{R}^{n},\|x\| \geq R\right\} \\
& \leq C \sup \left\{\left|x_{j}^{m} \cdot \widetilde{w}(x)\right| \mid x \in \mathbb{R}^{n},\|x\| \geq R\right\} \\
& \leq C M_{\widetilde{w}}\left(e_{j}, m\right) .
\end{aligned}
$$

3.2. Ostrowski's construction and weights on $\mathbb{R}$. We now discuss a well known type of construction, going back to Ostrowski [26], by which we assign an even weight on $\mathbb{R}$ to a non-negative logarithmically convex sequence. This in turn yields a method which allows us to pass from a given rapidly decreasing weight on $\mathbb{R}$ to an even rapidly decreasing majorant which has a certain minimal degree of regularity, while relevant other properties remain unchanged in the process. These two constructions (3.2) and (3.4) are important auxiliary tools in the study of weights.

Ostrowski's construction is as follows.

Suppose $\{a(m)\}_{m=0}^{\infty} \subset \mathbb{R}_{\geq 0}$ is logarithmically convex, i.e. $a(m)^{2} \leq a(m-1) a(m+$ 1 ) for $m \geq 1$. Define the associated weight $\widetilde{w}$ on $\mathbb{R}$ by

$$
\widetilde{w}(t)=\inf _{m \geq 0} \frac{a(m)}{|t|^{m}} \quad(t \in \mathbb{R})
$$

In case $t=0$ we interpret this expression (and similar ones in the sequel) as $a(0)$.

If $\{a(m)\}_{m=0}^{\infty}$ is not strictly positive (equivalently: $a(m)=0$ for all $m \geq 1$ ), then $\widetilde{w}(0)=a(0)$ and $\widetilde{w}(t)=0$ for $|t|>0$, implying that $M_{\widetilde{w}}(e, m)=a(m)$ for all integral $m \geq 0$.

If the sequence is strictly positive, then as a consequence of the logarithmical convexity we have the following local expression:

$$
\widetilde{w}(t)= \begin{cases}a(0) & \text { if } 0 \leq|t| \leq a(1) / a(0) \\ a(m) /|t|^{m} & \text { if } a(m) / a(m-1) \leq|t| \leq a(m+1) / a(m) \quad(m \geq 1) \\ 0 & \text { if }|t|>\sup _{m \geq 1} a(m) / a(m-1) .\end{cases}
$$

Some of the transition points can coincide, but there is no ambiguity. The value of $\sup _{m \geq 1} a(m) / a(m-1)$ can be finite or infinite; if it is finite then the value of $\widetilde{w}$ at $\sup _{m \geq 1} a(m) / a(m-1)$ is left open.

For each integral $m \geq 0$ the graph of $t \mapsto|t|^{m} \cdot \widetilde{w}(t)$ is evidently rather simple and from it one concludes that again $M_{\widetilde{w}}(e, m)=a(m)$ for all integral $m \geq 0$. We 
also note that $\widetilde{w}$ is continuous, non-increasing and strictly positive on the interval $\left(-\sup _{m \geq 1} a(m) / a(m-1), \sup _{m \geq 1} a(m) / a(m-1)\right)$ and that $s \mapsto-\log \widetilde{w}\left(e^{s}\right)$ is piecewise linear, non-decreasing and convex on $\left(-\infty, \log \sup _{m>1} a(m) / a(m-1)\right)$.

We record that for all non-negative logarithmically convex sequences $\{a(m)\}_{m=0}^{\infty}$ the series $\sum_{m=1}^{\infty} a(m)^{-1 / m}$ is divergent if and only if the associated weight $\widetilde{w}$ in (3.2) is quasi-analytic.

For the second construction, i.e. passing form a given rapidly decreasing weight $w$ on $\mathbb{R}$ to a more regular even rapidly decreasing majorant, we apply the first construction to the non-negative sequence $\left\{M_{w}(e, m)\right\}_{m=0}^{\infty}$ (which by Remark 3.5 is logarithmically convex) and obtain

$$
\widetilde{w}(t)=\inf _{m \geq 0} \frac{M_{w}(e, m)}{|t|^{m}} \quad(t \in \mathbb{R}) .
$$

It follows from the definitions that $\widetilde{w}$ is an even rapidly decreasing majorant of $w$. From the above discussion of (3.2) we see that $M_{\widetilde{w}}(e, m)=M_{w}(e, m)$ for all integral $m \geq 0$. In particular $\widetilde{w}$ is a quasi-analytic weight if and only if $w$ is. It is also obvious from the discussion of (3.3) that $\widetilde{w}$ has a certain minimal degree of regularity and a convexity property if supp $w \nsubseteq\{0\}$.

Although we will make no specific use of it, let us mention that in case we start with an arbitrary rapidly decreasing weight $w$ such that supp $w \nsubseteq\{0\}$, then $\sup _{m \geq 1} M_{w}(e, m) / M_{w}(e, m-1)=\Delta_{w}$, where $\Delta_{w}=\sup _{w(t) \neq 0}|t|$ is the possibly infinite diameter of the symmetric convex hull of the support of $w$. Therefore $\Delta_{\widetilde{w}}=\Delta_{w}$, so that the assignment $w \mapsto \widetilde{w}$ preserves this diameter in this case; this is therefore true for all rapidly decreasing non-zero weights $w$. If $\Delta_{w}<\infty$ then $\widetilde{w}(\Delta)=\lim _{s \uparrow \Delta} \sup _{|t| \geq s} w(t)=\inf _{0 \leq s<\Delta} \sup _{|t| \geq s} w(t)$. This determines the missing value in (3.3).

3.3. Quasi-analytic weights. In section we establish some basic characteristics of quasi-analytic weights and then proceed to classify such weights.

First of all, there is an obvious and important way to construct a weight $w^{\prime}$ on $\mathbb{R}^{n}$ from an even weight $w$ on $\mathbb{R}$ by putting $w^{\prime}(x)=w(\|x\|)$ for $x \in \mathbb{R}^{n}$. Then $M_{w^{\prime}}(v, m)=\|v\|^{m} M_{w}(e, m)$ for $v \in \mathbb{R}^{n}$ and all integral $m \geq 0$. This implies that for $w^{\prime}$ quasi-analyticity and quasi-analyticity with respect to all bases of $\mathbb{R}^{n}$ are both equivalent to the quasi-analyticity of $w$ on $\mathbb{R}$. As a consequence, if $w$ is quasianalytic on $\mathbb{R}$ then by Proposition 3.9 all weights on $\mathbb{R}^{n}$ majorized at infinity by $w^{\prime}$ are again quasi-analytic. To be more precise: by this proposition such minorants at infinity are quasi-analytic with respect to any basis of $\mathbb{R}^{n}$. It will become apparent from the classification of quasi-analytic weights on $\mathbb{R}$ and the explicit examples in Section 3.4 that the collection of such radial quasi-analytic weights on $\mathbb{R}^{n}$ and their minorants is in many practical situations already an interesting one to work with.

In its essence, the situation is nevertheless fundamentally different. Whenever $n \geq 2$, there exist quasi-analytic weights on $\mathbb{R}^{n}$ which are not obtainable in this way as minorants at infinity of (essentially) one-dimensional quasi-analytic weights. Indeed, we will show shortly that there exist weights which are quasi-analytic with respect to precisely one basis, up to scalar multiplication. These weights then clearly provide examples of such non-minorants at infinity.

This possibility of the existence of an essentially unique basis related to a particular element of $\mathcal{W}_{q a}$ has the same origin as the failure of $\mathcal{W}_{q a}$ to be closed under 
addition, a fact which was already mentioned in Proposition 3.7. To construct examples of these phenomena we need the following proposition.

Proposition 3.10. For $k \geq 2$ there exist strictly positive logarithmically convex sequences $\left\{a_{j}(m)\right\}_{m=0}^{\infty}$ for $j=1, \ldots, k$ such that

$$
\sum_{m=1}^{\infty} a_{j}(m)^{-1 / m}=\infty \quad(j=1, \ldots, k),
$$

but

$$
\sum_{m=1}^{\infty}\left(\max \left(a_{j_{1}}(m), a_{j_{2}}(m)\right)\right)^{-1 / m}<\infty \quad\left(1 \leq j_{1} \neq j_{2} \leq k\right) .
$$

Proof. Consider the convex function $f:[1, \infty) \mapsto \mathbb{R}$ defined by $f(x)=2 x \log x$. The idea is to construct convex functions $f_{j}:[1, \infty) \mapsto \mathbb{R}(j=1, \ldots, n)$ such that:

$$
\begin{cases}\max \left(f_{j_{1}}, f_{j_{2}}\right) & =f \quad\left(1 \leq j_{1} \neq j_{2} \leq k\right) ; \\ \sum_{m=1}^{\infty} e^{-f_{j}(m) / m} & =\infty\end{cases}
$$

Once this is accomplished, we can define $\log a_{j}(m)=f_{j}(m)$ for $j=1, \ldots, k$ and $m \geq 1$. Since it is obviously possible to define the $a_{j}(0)$ in addition, preserving the strictly positive and logarithmically convex properties of $\left\{a_{j}(m)\right\}_{m=1}^{\infty}$, this yields sequences satisfying (3.5) and (3.6).

The $f_{j}$ are constructed as partially "tangentialized" versions of $f$, as follows. Let $N_{1} \geq 1$ be an arbitrary integer. Consider the tangent $T_{1}$ to the graph of $f$ at $\left(N_{1}, f\left(N_{1}\right)\right)$. If $T_{1}(x)$ is the ordinate of a point at this tangent with abscissa $x$, then $T_{1}(x) / x$ trivially tends to a limit as $x \rightarrow \infty$. It is therefore possible to select integral $N_{1}^{\prime}>N_{1}$ such that $\sum_{m=N_{1}}^{N_{1}^{\prime}} \exp \left(-T_{1}(m) / m\right)>1$. Having done this, consider the other tangent $T_{1}^{\prime}$ to the graph of $f$ which passes through $\left(N_{1}^{\prime}, T_{1}\left(N_{1}^{\prime}\right)\right)$ and which is tangent to the graph of $f$ at, say, $\left(R_{1}, f\left(R_{1}\right)\right)$ for some real $R_{1}>N_{1}^{\prime}$. Then $f$ admits the possibility of "tangentialization" along the tangents $T_{1}$ and $T_{1}^{\prime}$ on the interval $I_{1}=\left[N_{1}, N_{1}^{\prime}\right] \cup\left[N_{1}^{\prime}, R_{1}\right]$ into a function $\widetilde{f}$, which is given by $\widetilde{f}(x)=f(x)$ for $x \in\left[1, N_{1}\right] \cup\left[R_{1}, \infty\right)$, by $\widetilde{f}(x)=T_{1}(x)$ for $x \in\left[N_{1}, N_{1}^{\prime}\right]$, and by $\widetilde{f}(x)=T_{1}^{\prime}(x)$ for $x \in\left[N_{1}^{\prime}, R_{1}\right]$. The point of this procedure is that $\sum_{m=N_{1}}^{N_{1}^{\prime}} \exp (-\widetilde{f}(m) / m)>1$ by construction, while $\tilde{f}$ is again convex as a consequence of our choice to work with tangents. By this inherited convexity, we can repeat this procedure indefinitely and determine a collection of possible tangentializations of $f$ on mutually disjoint intervals $I_{r}(r=1,2, \ldots)$, each interval decomposable as $I_{r}=\left[N_{r}, N_{r}^{\prime}\right] \cup\left[N_{r}^{\prime}, R_{r}\right]$ such that $\sum_{m=N_{r}}^{N_{r}^{\prime}} \exp \left(-T_{r}(m) / m\right)>1$, and with the property that a realization of this tangentialization of $f$ on any subcollection of the total collection of all intervals $I_{r}$ again yields a convex function on $[1, \infty)$. Therefore, if we let $f_{j}$ be defined by tangentializing $f$ on the subcollection of all intervals $I_{r}$ with $r=j \bmod k$, then the $f_{j}$ are convex. They satisfy the first equation in (3.7) since the $I_{r}$ are disjoint and the second equation because $\sum_{m=N_{r}}^{N_{r}^{\prime}} \exp \left(-f_{j}(m) / m\right)=\sum_{m=N_{r}}^{N_{r}^{\prime}} \exp \left(-T_{r}(m) / m\right)>1$ whenever $r=j \bmod k$.

Returning to our phenomena, let us first show that $\mathcal{W}_{q a}$ is not closed under addition. Select sequences as in the proposition for $k=2$ and apply the construction (3.2) to both sequences, obtaining even quasi-analytic weights $\widetilde{w}_{1,2}$ on $\mathbb{R}$. The 
canonical radial procedure (which is the identity if $\mathbb{R}^{n}=\mathbb{R}$ ) yields radial quasianalytic weights $\widetilde{w}_{1,2}^{\prime}$ on $\mathbb{R}^{n}$. Now since obviously

$$
\begin{aligned}
M_{\widetilde{w}_{1}^{\prime}+\widetilde{w}_{2}^{\prime}}(v, m) & \geq \max \left(M_{\widetilde{w}_{1}^{\prime}}(v, m), M_{\widetilde{w}_{2}^{\prime}}(v, m)\right) \\
& =\|v\|^{m} \max \left(M_{\widetilde{w}_{1}}(e, m), M_{\widetilde{w}_{2}}(e, m)\right) \\
& =\|v\|^{m} \max \left(a_{1}(m), a_{2}(m)\right)
\end{aligned}
$$

for all $v \in \mathbb{R}^{n}$ and all $m \geq 0$, (3.6) shows that $\widetilde{w}_{1}^{\prime}+\widetilde{w}_{2}^{\prime}$ is not quasi-analytic with respect to any basis. We have therefore found two weights on $\mathbb{R}^{n}$, each quasianalytic with respect to all bases and such that their sum is not quasi-analytic. In particular, $\mathcal{W}_{q a}$ is in all dimensions not closed under addition.

In order to show the possibility of the existence of a distinguished basis for a quasi-analytic weight on $\mathbb{R}^{n}(n \geq 2)$, take strictly positive logarithmically convex sequences $\left\{a_{j}(m)\right\}_{m=0}^{\infty}$ for $j=1, \ldots, n$ satisfying (3.5) and (3.6) and obtain even quasi-analytic weights $\widetilde{w}_{j}(j=1, \ldots, n)$ on $\mathbb{R}$ by (3.2). Define $w: \mathbb{R}^{n} \mapsto \mathbb{R}_{\geq 0}$ as the tensor product $w(x)=\prod_{j=1}^{n} \widetilde{w}_{j}\left(x_{j}\right)$. It is immediate that $w$ is quasi-analytic with respect to the standard basis. Furthermore, if $v=\sum_{j=1}^{n} \lambda_{j} e_{j} \in \mathbb{R}^{n}$, then consideration of the restriction of $w$ to the union of each possible pair of coordinate axes shows that for $1 \leq j_{1} \neq j_{2} \leq n$ and integral $m \geq 0$ :

$$
M_{w}(v, m) \geq \max \left(a_{j_{1}}(m), a_{j_{2}}(m)\right)\left(\min \left(\left|\lambda_{j_{1}}\right|,\left|\lambda_{j_{2}}\right|\right)\right)^{m} \min \left(a_{j_{1}}(0), a_{j_{2}}(0)\right) \prod_{j \neq j_{1}, j_{2}} a_{j}(0) .
$$

Therefore, if $w$ is quasi-analytic with respect to a basis containing $v$ then by (3.6) we must have $\min \left(\left|\lambda_{j_{1}}\right|,\left|\lambda_{j_{2}}\right|\right)=0$ for all $j_{1} \neq j_{2}$. This implies that the standard basis is the only possible choice, up to scaling.

This construction concludes our discussion of basic characteristics of quasi-analytic weights and we will now start the classification. The first step is a reduction to $\mathbb{R}$, for which we need a preparatory result.

Proposition 3.11 (cf. [33, 23]). Let $w$ be a weight on $\mathbb{R}^{n}$. If $w$ is quasi-analytic with respect to the basis $\left\{v_{1}, \ldots, v_{n}\right\}$ then $w^{\nu}$ is also quasi-analytic with respect to this basis for all $\nu>0$.

Proof. By Lemma 3.6 we may assume that $w$ is standard quasi-analytic. We may obviously also assume that $\|w\|_{\infty}=1$. Note that for all $1 \leq j \leq n$, all strictly positive integers $p$ and all integral $m \geq 1$ we have trivially

$$
M_{w^{1 / p}}\left(e_{j}, m\right)^{1 / m}=M_{w}\left(e_{j}, m p\right)^{1 / m p} .
$$

The third part of Lemma 3.4 and the second part of Lemma 3.3 then imply that $w^{1 / p}$ is standard quasi-analytic for all strictly positive integral $p$. Since $w$ tends to zero at infinity, we have $w^{\nu} \leq w^{1 / p}$ at infinity whenever $p>1 / \nu$. An appeal to Proposition 3.9 finishes the proof.

Proposition 3.12. The weight $w$ is a standard quasi-analytic weight on $\mathbb{R}^{n}$ if and only if there exist even quasi-analytic weights $w_{1}, \ldots, w_{n}$ on $\mathbb{R}$ such that $w(x) \leq$ $\prod_{j=1}^{n} w_{j}\left(x_{j}\right)$ for all $x \in \mathbb{R}^{n}$.

Proof. The "if"-part is obvious. As to the converse, first note that from Remark 3.5 we know that $\left\{M_{w^{1 / n}}\left(e_{j}, m\right)\right\}_{m=0}^{\infty}$ is a non-negative logarithmically convex sequence 
for each $j=1, \ldots, n$. We can therefore apply construction (3.2) to each of these sequences and define

$$
\widetilde{w}_{j}(t)=\inf _{m \geq 0} \frac{M_{w^{1 / n}}\left(e_{j}, m\right)}{|t|^{m}} \quad(t \in \mathbb{R}) .
$$

Now since $w$ is standard quasi-analytic on $\mathbb{R}^{n}$ we conclude from Proposition 3.11 that $\sum_{m=1}^{\infty} M_{w^{1 / n}}\left(e_{j}, m\right)^{-1 / m}=\infty(j=1, \ldots, n)$. As noted when discussing construction (3.2), this implies that each $\widetilde{w}_{j}$ is an (obviously even) quasi-analytic weight on $\mathbb{R}$. From the definitions one sees that $w^{1 / n}(x) \leq \widetilde{w}_{j}\left(x_{j}\right)$ for all $x \in \mathbb{R}^{n}$ and $j=1, \ldots, n$. The $\widetilde{w}_{j}$ are therefore as required.

Since the orbit of a quasi-analytic weight under the affine group contains a standard quasi-analytic weight, Proposition 3.12 shows that the classification of quasi-analytic weights on $\mathbb{R}^{n}$ is now reduced to quasi-analytic weights on $\mathbb{R}$. The key in that case is the following lemma, in which for the real line the defining property (3.1) is linked to a classical condition. This connection seems to have gone largely unnoticed although there are similarities with 19, proof of Theorem 2], under additional regularity conditions on the weight.

Lemma 3.13. Suppose $w$ is a weight on $\mathbb{R}$ for which there exists $R>0$ such that $w(t)=w(-t)>0$ for $|t|>R$ and additionally $s \mapsto-\log w\left(e^{s}\right)$ is convex on $(\log R, \infty)$. Then $w$ is a quasi-analytic weight if and only if

$$
\int_{R}^{\infty} \frac{\log w(t)}{1+t^{2}}=-\infty
$$

Proof. The basic argument and the key inequalities that we will employ go (at least) back to Horváth [13] (alternatively see [16, p. 98]) and we refer the reader to these sources for details.

Leaving the lemma aside for the moment, let us consider the following situation.

Let $R \geq 1$ and suppose that $w:(R, \infty) \mapsto \mathbb{R}_{>0}$ is rapidly decreasing such that $\psi(s)=-\log w\left(e^{s}\right)$ is convex and strictly increasing on $(\log R, \infty)$.

For all real $\rho \geq 0$ (the lower bound stems from the hypothesis that $\psi$ is strictly increasing) put

$$
M(\rho)=\sup _{t>R} t^{\rho} w(t)
$$

Then the convexity of $\psi$ implies that $w$ can be retrieved on $(R, \infty)$ as

$$
w(t)=\inf _{\rho \geq 0} \frac{M(\rho)}{t^{\rho}} \quad(t>R)
$$

Define $q:[0, \infty) \mapsto \mathbb{R}_{\geq 0}$ by

$$
q(t)=\inf _{m=0,1,2, \ldots} \frac{M(m)}{t^{m}} \quad(t \geq 0) .
$$

In view of (3.10), the restriction of $q$ to $(R, \infty)$ can be viewed as an approximation of $w$. Using that $R \geq 1$ one then derives the key inequalities:

$$
w(t) \leq q(t) \leq t w(t) \quad(t>R)
$$

Since $q$ is non-increasing on $[0, \infty)$ and $w$ is strictly positive on $(R, \infty)$, the first of these inequalities implies that $\log q$ is bounded from below on any compact subset 
of $[0, \infty)$. Evidently $\log q$ is bounded from above by $\log M(0)$. Therefore, using (3.11) we conclude that

$$
\int_{R}^{\infty} \frac{\log w(t)}{1+t^{2}}=-\infty
$$

if and only if

$$
\int_{0}^{\infty} \frac{\log q(t)}{1+t^{2}}=-\infty
$$

since the term $\log t$ is immaterial, as is for $\log q$ the integral from 0 to $R$.

On the other hand, the first part of Lemma 3.4 implies that the $M(m)$ form a logarithmically convex sequence, which is obviously strictly positive. Therefore a standard equivalence [28, Thm. 19.11], relevant in the context of quasi-analytic classes, can be used to conclude that $\sum_{m=1}^{\infty} M(m)^{-1 / m}=\infty$ if and only if (3.13) holds.

Both equivalences together therefore yield as conclusion that $\sum_{m=1}^{\infty} M(m)^{-1 / m}=$ $\infty$ if and only if (3.12) holds.

Returning to the lemma proper now, suppose that $w$ is as in the lemma and that (3.8) holds. Introduce $\psi(s)=-\log w\left(e^{s}\right)$ on $(\log R, \infty)$ and choose any $s_{0} \in(R, \infty)$. The convexity of $\psi$ implies that $\left(\psi(s)-\psi\left(s_{0}\right)\right) /\left(s-s_{0}\right)$ is non-decreasing as a function of $s \in(\log R, \infty)$. If this quotient remains bounded by $M \in \mathbb{R}$ when $s \rightarrow \infty$, then $w(t) \geq C t^{-M}$ for some $C>0$ and all $t>\exp s_{0}$, implying that (3.8) is violated. Hence the quotient tends to infinity, which implies that $w$ is rapidly decreasing on $(R, \infty)$. Since the quotient is, in particular, eventually strictly positive, $\psi$ is eventually strictly increasing. Hence we may assume, by enlarging $R$ if necessary, that this is actually the case on $(\log R, \infty)$. Evidently we can also assume $R \geq 1$.

We are then in the situation as described above and conclude from (3.12) that $\sum_{m=1}^{\infty} M(m)^{-1 / m}=\infty$, where $M(m)=\sup _{t>R} t^{m} w(t)$. Now since $w$ has unbounded support, the fact that $w$ is even and the argument in the proof of Proposition 3.9 show that $\left\|t^{m} \cdot w\right\|_{\infty}$ is equal to $M(m)$ for all sufficiently large $m$. We conclude that $w$ is a quasi-analytic weight, as was to be shown.

Conversely, if $w$ is a quasi-analytic weight as in the lemma, let us show that (3.8) holds. We again introduce $\psi(s)=-\log w\left(e^{s}\right)$ on $(\log R, \infty)$ and choose $s_{0} \in$ $(\log R, \infty)$. This time the quotients $\left(\psi(s)-\psi\left(s_{0}\right)\right) /\left(s-s_{0}\right)$ can not remain bounded as $s \rightarrow \infty$ since this would imply that $w \notin \mathcal{W}_{\infty}$, contradicting Lemma 3.6. Hence again we may assume, by enlarging $R$ if necessary, that $\psi$ is strictly increasing on $(\log R, \infty)$ for some $R \geq 1$, again placing us in the situation as described above. Reversing the arguments in the previous paragraph then shows that (3.12) holds.

We briefly review some well known facts on majorants in view of the next theorem.

As a preparation, suppose $w^{\prime}$ is an even weight on $\mathbb{R}$ such that $s \mapsto-\log w^{\prime}\left(e^{s}\right)$ is convex on $\mathbb{R}$, where we use the convention that $\log 0=-\infty$. Then, if $w^{\prime}\left(t_{j}\right)=0$ for some sequence $\left\{t_{j}\right\}_{j=1}^{\infty}$ with $t_{j} \rightarrow \infty$, the convexity of $s \mapsto-\log w^{\prime}\left(e^{s}\right)$ implies that actually $w^{\prime}(t)=0$ for all sufficiently large $|t|$. We see that any even $w^{\prime}$ with this convexity property is for sufficiently large absolute value of the argument either strictly positive or identically zero. 
Next, if $w$ is a weight on $\mathbb{R}$, consider the set $\overline{\mathcal{W}}_{w}$ of all even weights $\widetilde{w}$ on $\mathbb{R}$ that are majorants of $w$ such that $s \mapsto-\log \widetilde{w}\left(e^{s}\right)$ is convex on $\mathbb{R}$. Note that $\overline{\mathcal{W}}_{w}$ contains at least the sufficiently large constants, so that we may define $\bar{w}(x)=$ $\inf _{w^{\prime} \in \overline{\mathcal{W}}_{w}} w^{\prime}(x)$. Then $\bar{w} \in \overline{\mathcal{W}}_{w}$ and by the previous paragraph $\bar{w}$ is for sufficiently large absolute value of the argument either strictly positive or identically zero.

The quasi-analytic weights on the real line are now classified in the following theorem.

Theorem 3.14. Let $w$ be a weight (i.e. an arbitrary non-negative bounded function) on $\mathbb{R}$. Then the following are equivalent:

1. $w$ is a quasi-analytic weight, i.e.

$$
\sum_{m=1}^{\infty}\left\|x^{m} \cdot w(x)\right\|_{\infty}^{-1 / m}=\infty
$$

2. For all $R>0$ :

$$
\int_{R}^{\infty} \frac{\log \bar{w}(t)}{1+t^{2}}=-\infty
$$

3. There exist a weight $\widetilde{w}$ on $\mathbb{R}$ and $R>0$ such that $w(t) \leq \widetilde{w}(t)$ and $\widetilde{w}(t)=$ $\widetilde{w}(-t)>0$ both hold for $|t|>R, s \mapsto-\log \widetilde{w}\left(e^{s}\right)$ is convex on $(\log R, \infty)$ and

$$
\int_{R}^{\infty} \frac{\log \widetilde{w}(t)}{1+t^{2}}=-\infty
$$

4. There exist a strictly positive $\widetilde{w} \in \overline{\mathcal{W}}_{w}$ of class $C^{\infty}$ and $\epsilon>0$ such that $\widetilde{w}$ is constant on $[0, \epsilon]$ and strictly decreasing on $[\epsilon, \infty)$, and such that

$$
\int_{0}^{\infty} \frac{\log \widetilde{w}(t)}{1+t^{2}}=-\infty
$$

5. There exists $C \geq 0$ and a non-decreasing non-negative function $\rho:[0, \infty) \mapsto$ $\mathbb{R}_{\geq 0}$ of class $C^{\infty}$, which is equal to zero on $[0, \epsilon]$ for some $\epsilon>0$, such that

$$
w(t) \leq C \exp \left(-\int_{0}^{|t|} \frac{\rho(s)}{s} d s\right) \quad(t \in \mathbb{R})
$$

and

$$
\int_{0}^{\infty} \frac{\rho(s)}{1+s^{2}} d s=\infty .
$$

Proof. We first show that (1) implies (4) in case $w$ has unbounded support, i.e. in case there exist $t$ such that $w(t) \neq 0$ with $|t|$ arbitrarily large. We apply a slight variation of the construction (3.4) to the strictly positive logarithmically convex sequence $\left\{M_{w}(e, m)\right\}_{m=0}^{\infty}$ and define $w^{\prime}$ by putting

$$
w^{\prime}(t)=2 \inf _{m \geq 0} \frac{M_{w}(e, m)}{|t|^{m}} \quad(t \in \mathbb{R}) .
$$

As noted when discussing (3.2), (3.4) and (3.3), $w^{\prime} / 2$ is an even quasi-analytic weight majorizing $w$. The same then holds for $w^{\prime}$. Hence $w^{\prime}$ has unbounded support; since $w^{\prime}$ is evidently non-increasing on $[0, \infty)$, it becomes apparent that $w^{\prime}$ is strictly positive. This implies that the third possibility in (3.3) does not occur and that $w^{\prime}$ is therefore continuous. We also conclude from (3.3) that $s \mapsto-\log w^{\prime}\left(e^{s}\right)$ is convex on $(-\infty, \infty)$. The graph of this map is in fact particularly simple, since it 
is connected and consists of a horizontal half-line, followed by a sequence of line segments with increasing strictly positive slope.

We therefore conclude from Lemma 3.13 that

$$
\int_{0}^{\infty} \frac{\log w^{\prime}(t)}{1+t^{2}}=-\infty
$$

Hence $w^{\prime}$ already has all the required properties, except that it is only piecewise smooth. To improve this, note that the piecewise linear character of the graph of $s \mapsto-\log w^{\prime}\left(e^{s}\right)$ shows that it is possible to smooth this graph in a convex fashion while still remaining below the graph of $s \mapsto-\log \left(w^{\prime}\left(e^{s}\right) / 2\right)$. We choose any such convex smoothing, leaving the original graph horizontal on $(-\infty, r)$ for some $r \in \mathbb{R}$, and declare the smooth version to be the graph of $s \mapsto-\log \widetilde{w}\left(e^{s}\right)$, thus defining $\widetilde{w}$ on $(0, \infty)$. Then $\widetilde{w}$ is on $(0, \infty)$ a majorant of $w^{\prime} / 2$ by construction and evidently of class $C^{\infty}$. Since $\widetilde{w}$ equals (unchanged) the constant $2 M_{w}(e, 0)$ on $(0, \exp r)$, it is evident that $\widetilde{w}$ can be extended to an even $C^{\infty}$ weight on $\mathbb{R}$ which then, since $\widetilde{w}(0)=2 M_{w}(e, 0)>M_{w}(e, 0)=w^{\prime}(0) / 2$ and since $w^{\prime} / 2$ is even, is a majorant of $w^{\prime} / 2$ on $\mathbb{R}$. We already observed that $w^{\prime} / 2 \geq w$, so $\widetilde{w}$ is a majorant of $w$. By construction $\widetilde{w} \leq w^{\prime}$, so the relevant integral involving $\widetilde{w}$ is divergent. Therefore $\widetilde{w}$ has all the required properties. This shows that (1) implies (4) if $w$ has unbounded support.

In case $w$ has bounded support, we choose $C>0$ such that $C \exp (-|t|)$ majorizes $w$ globally. By the previous result there is a suitable majorant $\widetilde{w}$ for $C \exp (-|t|)$, hence for $w$. This shows that (1) implies (4) in all cases.

The equivalence of (4) and (5) is matter of restating, using (2.1) to define $\rho$ in terms of $\widetilde{w}$ and vice versa. One reverses the order of integration in the resulting double integral; details are left to the reader.

It is trivial that (4) implies (3).

To see that (3) implies (1), note that Lemma 3.13 asserts that $\widetilde{w}$ is a quasianalytic weight. Then by Proposition 3.9 the same is true for $w$.

We have shown so far that (1), (3), (4) and (5) are equivalent. If $\widetilde{w}$ is as in (4) note that $\bar{w} \leq \widetilde{w}$, so $(2)$ follows.

Finally, if (2) holds, first suppose that $\bar{w}(t)=0$ for all sufficiently large $|t|$. Then $w$ has bounded support and (1) holds. In the remaining case $\bar{w}$ is strictly positive for sufficiently large absolute value of the argument, so that $\bar{w}$ satisfies the conditions for $\widetilde{w}$ in (3) if $R$ is sufficiently large. Thus (1) follows once more.

Remark 3.15. The various conditions in the proposition each cover different aspects. The first is the relevant one for the quasi-analytic property of certain transforms, as will become apparent in Section 1 . The second condition establishes the link between the notion of a quasi-analytic weight and regularization. The requirement in (2) that $R$ is arbitrary is necessary to avoid undue influence of an interval on which $\bar{w}$ vanishes identically. The third condition aims at the practice since it provides a positive criterion for a given weight which is easier to apply than the other conditions in the proposition. We will encounter a negative criterion Proposition 3.17. With respect to the fourth, let us note that there is a continuous injection $\mathcal{O}_{\widetilde{w}} \hookrightarrow \mathcal{O}_{w}$ whenever $\widetilde{w}$ and $w$ are arbitrary weights such that $\widetilde{w}$ majorizes $w$. Closure results in $\mathcal{O}_{\widetilde{w}}$ then transfer to $\mathcal{O}_{w}$ by Lemma 1.1; the implication of (4) is therefore that, although we made no such assumptions to start with, one can in many instances in fact assume that a quasi-analytic weight is 
strictly positive on compact sets (which is an important property) and smooth. Theorem 3.22 below is concerned with possibilities for such improvements for the various classes of weights. The fifth condition enables one to write down whole families of quasi-analytic weights. The collection of all smooth functions $\rho$ as in this part is apparently sufficient to completely describe the quasi-analytic weights on the real line. Note that the holomorphic weights are retrieved in the special case where $\rho$ is eventually linear.

The classification of quasi-analytic weights in arbitrary dimension is now a matter of combining previous results.

Theorem 3.16. Let $w$ be a weight (i.e. an arbitrary non-negative bounded function) on $\mathbb{R}^{n}$. Then the following are equivalent:

1. $w$ is a quasi-analytic weight, i.e. there exists a basis $\left\{v_{1}, \ldots, v_{n}\right\}$ of $\mathbb{R}^{n}$ such that

$$
\sum_{m=1}^{\infty}\left\|\left(v_{j}, x\right)^{m} \cdot w(x)\right\|_{\infty}^{-1 / m}=\infty \quad(1 \leq j \leq n) .
$$

2. There exist an affine transformation $A$ and weights $w_{j}(j=1, \ldots, n)$ on $\mathbb{R}$, each satisfying the five equivalent conditions in Theorem 3.14 and such that

$$
w(A x) \leq \prod_{j=1}^{n} w_{j}\left(x_{j}\right)
$$

for all $x \in \mathbb{R}^{n}$ with $\|x\|$ sufficiently large.

3. There exists a quasi-analytic weight $\widetilde{w}$ on $\mathbb{R}^{n}$ of class $C^{\infty}$ such that $w(x) \leq$ $\widetilde{w}(x)$ and $\widetilde{w}(x)=\widetilde{w}(-x)$ for all $x \in \mathbb{R}^{n}$ and such that $\inf _{x \in K} \widetilde{w}(x)>0$ for all non-empty compact subsets $K$ of $\mathbb{R}^{n}$.

Furthermore, if the first statement holds for a basis $\left\{v_{1}, \ldots, v_{n}\right\}$, then the second statement holds for any $A$ with linear component $A_{0}$ defined by $A_{0}^{t} v_{j}=e_{j}(j=$ $1, \ldots, n)$. Conversely, if the second statement holds and $A_{0}$ is the linear component of $A$, then the first statement holds for the basis $\left\{v_{1}, \ldots, v_{n}\right\}$ defined by $A_{0}^{t} v_{j}=$ $e_{j}(j=1, \ldots, n)$. If the first statement holds for a basis $\left\{v_{1}, \ldots, v_{n}\right\}$, then the weight $\widetilde{w}$ in the third statement can be chosen to be quasi-analytic with respect to this same basis.

Proof. Assuming (1), Proposition 3.7 yields a linear $A \in$ Aff such that $A^{-1} w$ is standard quasi-analytic. By Proposition $3.12 A^{-1} w$ is then majorized by the tensor product of quasi-analytic weights on $\mathbb{R}$. Each of these satisfies all five equivalent conditions in Theorem 3.14; hence (1) implies (2). Assuming (2) we note that the tensor product is a quasi-analytic weight on $\mathbb{R}^{n}$ by Theorem 3.14 and Proposition 3.12. By Proposition 3.9 we conclude that $A^{-1} w$ is quasi-analytic and the same is then true for $w$ by Proposition 3.7. Hence (2) implies (1).

Assuming (1) we conclude as above that there exists a linear $A \in$ Aff such that $A^{-1} w$ is majorized by the tensor product of quasi-analytic weights on $\mathbb{R}$. Majorizing each of these in turn as in the fifth part of Theorem 3.14 we conclude that there exists a standard quasi-analytic weight $w^{\prime}$ on $\mathbb{R}^{n}$ of class $C^{\infty}$ such that $A^{-1} w(x) \leq$ $w^{\prime}(x)$ and $w^{\prime}(x)=w^{\prime}(-x)$ for all $x \in \mathbb{R}^{n}$ and such that $\inf _{x \in K} w^{\prime}(x)>0$ for all non-empty compact subsets $K$ of $\mathbb{R}^{n}$. Therefore $A w^{\prime}$ satisfies the requirements for $\widetilde{w}$ and (1) implies (3). It is trivial that (3) implies (1).

The additional statements on the bases follow from Lemma 3.6 . 
The following proposition elaborates on quasi-analytic weights on the real line. Note that the first part of the proposition yields a negative criterion for quasianalyticity of weights on $\mathbb{R}$. The example in the third part shows that the necessary condition in the first part is not sufficient and that the symmetry assumption in the second statement is necessary.

Proposition 3.17. Let $w$ be a weight on $\mathbb{R}$.

1. If $w$ is quasi-analytic and Lebesgue measurable, then for all $R>0$ we have

$$
\int_{R}^{\infty} \frac{\log w(t)}{1+t^{2}}=\int_{-\infty}^{-R} \frac{\log w(t)}{1+t^{2}}=-\infty .
$$

2. If $w$ is even and such that $s \mapsto-\log w\left(e^{s}\right)$ is convex on $\mathbb{R}$, then $w$ is quasianalytic if and only if

$$
\int_{R}^{\infty} \frac{\log w(t)}{1+t^{2}}=-\infty
$$

for all $R>0$.

3. There exists a strictly positive weight $w$ on $\mathbb{R}$ such that $s \mapsto-\log w\left(e^{s}\right)$ and $s \mapsto-\log w\left(-e^{s}\right)$ are both convex on $\mathbb{R}$ and such that

$$
\int_{R}^{\infty} \frac{\log w(t)}{1+t^{2}}=\int_{-\infty}^{-R} \frac{\log w(t)}{1+t^{2}}=-\infty
$$

for all $R>0$, but which is not quasi-analytic.

Proof. In the first statement, the divergence of the integrals is implied by the second part of Theorem 3.14 since $w(t) \leq \bar{w}(|t|)$ for all $t$. The second statement is immediate from again the second part of Theorem 3.14 since now $w=\bar{w}$.

As to the third statement, Proposition 3.10 provides two strictly positive logarithmically convex sequences $\left\{a_{j}(m)\right\}_{m=0}^{\infty}$ for $j=1,2$ such that

$$
\sum_{m=1}^{\infty} a_{j}(m)^{-1 / m}=\infty \quad(j=1,2)
$$

but

$$
\sum_{m=1}^{\infty}\left(\max \left(a_{1}(m), a_{2}(m)\right)\right)^{-1 / m}<\infty .
$$

We apply (3.2) to both sequences, obtaining even quasi-analytic weights $w_{1,2}$ on $\mathbb{R}$ with the property that $M_{w_{1,2}}(e, m)=a_{1,2}(m)$ for all integral $m \geq 0$ and such that the maps $s \mapsto-\log w_{1,2}\left(e^{s}\right)$ are both convex on $\mathbb{R}$. The first part of the present proposition shows that

$$
\int_{R}^{\infty} \frac{\log w_{1}(t)}{1+t^{2}}=\int_{-\infty}^{-R} \frac{\log w_{2}(t)}{1+t^{2}}=-\infty .
$$

for all $R>0$. Choose $w=1_{\mathbb{R}_{<0}} w_{1}+1_{\mathbb{R}_{>0}} w_{2}+1_{\{0\}}$. Then evidently $M_{w}(e, m)=$ $\max \left(M_{w_{1}}(e, m), M_{w}(e, m)\right)=\max \left(a_{1}(m), a_{2}(m)\right)$ for all $m \geq 1$, so that $w$ is an example as required.

We finally note that we have the following necessary condition for a weight to be quasi-analytic in arbitrary dimension, again providing us with a negative criterion. 
Corollary 3.18. Let $w$ be a quasi-analytic weight on $\mathbb{R}^{n}$. If $x, y \in \mathbb{R}^{n}$ are such that $y \neq 0$ and such that $t \mapsto w(x+t y)$ is Lebesgue measurable on $\mathbb{R}$, then for all $R>0$ we have

$$
\int_{R}^{\infty} \frac{\log w(x+t y)}{1+t^{2}}=\int_{-\infty}^{-R} \frac{\log w(x+t y)}{1+t^{2}}=-\infty .
$$

Proof. Since $\mathcal{W}_{q a}$ is invariant under translations by Lemma 3.6, we may assume $x=0$. Let $\widetilde{w}(t)=w(t y)$, then $\widetilde{w}$ is a weight on $\mathbb{R}$. Suppose $w$ is quasi-analytic with respect to the basis $\left\{v_{1}, \ldots, v_{n}\right\}$. Select $v_{j}$ such that $\left(v_{j}, y\right) \neq 0$. Since $M_{\widetilde{w}}(e, m)=\left|\left(v_{j}, y\right)\right|^{-m} \sup \left\{\left|\left(v_{j}, t y\right)\right|^{m} \cdot w(t y) \mid t \in \mathbb{R}\right\} \leq\left|\left(v_{j}, y\right)\right|^{-m} M_{w}\left(v_{j}, m\right)$ for all $m \geq 0, \widetilde{w}$ is a quasi-analytic weight on $\mathbb{R}$. We now apply the first part of Proposition 3.17 .

3.4. Examples of quasi-analytic weights. On basis of the previous results we can now determine explicit examples and counterexamples of quasi-analytic weights on $\mathbb{R}^{n}$. We concentrate mainly on minorants and majorants of radial weights.

Proposition 3.19. Suppose $R_{0}>0, C \geq 0$ and a non-decreasing function $\rho$ : $\left(R_{0}, \infty\right) \mapsto \mathbb{R}_{\geq 0}$ of class $C^{1}$ are such that

$$
\int_{R_{0}}^{\infty} \frac{\rho(s)}{s^{2}} d s=\infty
$$

If $w$ is a weight such that

$$
w(x) \leq C \exp \left(-\int_{R_{0}}^{\|x\|} \frac{\rho(s)}{s}\right) d s
$$

whenever $\|x\| \geq R_{0}$, then the weight $w$ is quasi-analytic with respect to all bases of $\mathbb{R}^{n}$.

Indeed, with the aid of the third part of Theorem 3.14 and the discussion of (2.1) one concludes that such $w$ is majorized by the radial extension of an even quasi-analytic weight on $\mathbb{R}$; it is therefore a quasi-analytic weight on $\mathbb{R}^{n}$.

The first part of the following result exhibits families of examples of quasianalytic weights in terms of elementary functions, incorporating the holomorphic weights in a natural way. The second part shows that the first part is in a sense sharp.

Proposition 3.20. Define repeated logarithms by $\log _{0} t=t$ and, inductively, for $j \geq 1$, by $\log _{j} t=\log \left(\log _{j-1} t\right)$, where $t$ is assumed to be sufficiently large for the definition to be meaningful in the real context. For $j=0,1,2 \ldots$ let $a_{j}>0$ and let $p_{j} \in \mathbb{R}$ be such that $p_{j}=0$ for all sufficiently large $j$. Put $j_{0}=\min \{j=0,1,2, \ldots \mid$ $\left.p_{j} \neq 1\right\}$. Let $C>0$ and suppose $w: \mathbb{R}^{n} \mapsto \mathbb{R}_{\geq 0}$ is bounded. Then:

1. If $p_{j_{0}}<1$ and

$$
w(x) \leq C \exp \left(-\|x\|^{2}\left(\prod_{j=0}^{\infty} \log _{j}^{p_{j}} a_{j}\|x\|\right)^{-1}\right)
$$

for all sufficiently large $\|x\|$, then the weight $w$ is quasi-analytic with respect to all bases of $\mathbb{R}^{n}$. 
2. If $p_{j_{0}}>1$ and

$$
w(x) \geq C \exp \left(-\|x\|^{2}\left(\prod_{j=0}^{\infty} \log _{j}^{p_{j}} a_{j}\|x\|\right)^{-1}\right)
$$

for all sufficiently large $\|x\|$, then $w$ is not a quasi-analytic weight on $\mathbb{R}^{n}$.

Proof. It is sufficient to prove this in one variable, in view of the properties of the radial extension procedure. In that case, the second statement follows immediately from Proposition 3.9 and Proposition 3.17. The first statement follows from the third part of Theorem 3.14 after some computation and asymptotics to check that the relevant function is eventually convex.

Concentrating on the radial case, a weight on $\mathbb{R}^{n}$ which for some $C, a_{0}, a_{1}, a_{2}, \ldots>$ 0 and all sufficiently large $\|x\|$ is majorized by whichever of the expressions

$$
\begin{aligned}
& C \exp \left(-\frac{\|x\|^{1-\nu}}{a_{0}}\right), \\
& C \exp \left(-\frac{\|x\|}{a_{0}\left(\log a_{1}\|x\|\right)^{1+\nu}}\right), \\
& C \exp \left(-\frac{\|x\|}{a_{0} \log a_{1}\|x\|\left(\log \log a_{2}\|x\|\right)^{1+\nu}}\right), \\
& \ldots
\end{aligned}
$$

is in $\mathcal{W}_{q a}$ if $\nu \leq 0$. Note that the first family of majorants is in $\mathcal{W}_{\text {hol }}$, but that the others are not. Complementary, a weight which is for some $\nu>0$ minorized at infinity by whichever of these expressions is not a quasi-analytic weight.

For the sake of completeness we mention that explicit non-radial examples of quasi-analytic weights on $\mathbb{R}^{n}$ in terms of elementary functions can be obtained as tensor products of quasi-analytic weights on $\mathbb{R}$ taken from the above proposition. All minorants at infinity of such weights are then again quasi-analytic weights on $\mathbb{R}^{n}$.

3.5. Improving the spaces $\mathcal{O}_{w}$. In this section we observe that many spaces $\mathcal{O}_{w}$ are Fréchet and show that in a sense these well behaved spaces are ubiquitous. This is an important fact which we use in Section 1 to invoke vector valued integration and in Section 5 to show the redundancy of a hypothesis of continuity. We also note that we can combine this completeness with an improvement of some regularity properties of the weight, without leaving the class of weights under consideration.

Proposition 3.21. Let $w$ be a weight such that for all non-empty compact subsets $K$ of $\mathbb{R}^{n}$ there exists $C_{K}>0$ such that $K \subset \overline{\left\{x \in \mathbb{R}^{n} \mid w(x)>C_{K}\right\}}$. Then $\mathcal{O}_{w}$ is a Fréchet space.

Proof. The hypothesis implies that the complement of the zero locus of $w$ is dense, hence $\mathcal{O}_{w}$ is Hausdorff by Remark 3.1 and only completeness remains to be checked. Let $\left\{f_{n}\right\}_{n=1}^{\infty}$ be a Cauchy sequence in $\mathcal{O}_{w}$. Let $K$ be an arbitrary non-empty compact subset of $\mathbb{R}^{n}$ and select $C_{K}>0$ such that $K \subset \overline{A_{K}}$ where $A_{K}=\{x \in$ $\left.\mathbb{R}^{n} \mid w(x)>C_{K}\right\}$. For all fixed $\alpha \in \mathbb{N}^{n}$, the restrictions of the $\partial^{\alpha} f_{n}$ to $A_{K}$ are then bounded on $A_{K}$ and are in fact a Cauchy-sequence under the supremum 
norm on the space of bounded functions on $A_{K}$. The analogous statement is then by continuity true for the restrictions to $\overline{A_{K}} \supset K$. We conclude that the $f_{n}$ are a Cauchy sequence in $C^{\infty}\left(\mathbb{R}^{n}\right)$, the latter space being supplied with the usual Fréchet topology of uniform convergence of all derivatives on all compact sets. A routine verification shows that the limit in $C^{\infty}\left(\mathbb{R}^{n}\right)$ is in fact an element of $\mathcal{O}_{w}$ and that the sequence converges to this limit in the topology of $\mathcal{O}_{w}$.

Theorem 3.22. $\quad$ 1. Let $w$ and $\widetilde{w}$ be weights such that

(a) $w(x) \leq \widetilde{w}(x)$ for all $x \in \mathbb{R}^{n}$, and

(b) $\widetilde{w}$ is separated from zero on compact sets.

Then $\mathcal{O}_{\widetilde{w}}$ is a Fréchet space which is identified with a subspace of $\mathcal{O}_{w}$. The corresponding injection $\mathcal{O}_{\widetilde{w}} \hookrightarrow \mathcal{O}_{w}$ is continuous, as is the injection $\mathcal{O}_{\widetilde{w}} \hookrightarrow$ $C^{\infty}\left(\mathbb{R}^{n}\right)$.

2. If $w \in \mathcal{W}_{d}$ for some $0 \leq d<\infty$ (resp. $w \in \mathcal{W}_{\text {hol }}$ ) there exists a radial $\widetilde{w} \in \mathcal{W}_{d}$ (resp. a radial $\widetilde{w} \in \overline{\mathcal{W}}_{\text {hol }}$ with $\tau_{\widetilde{w}}=\tau_{w}$ ) which satisfies the requirements under (1a) and (1b). If $w \in \mathcal{W}_{\infty}$ (resp. $\left.w \in \mathcal{W}\right)$, then there exists $\widetilde{w} \in \mathcal{W}_{\infty}$ (resp. $w \in \mathcal{W}$ ) which is of class $C^{\infty}$, radial, non-increasing on each ray emanating from the origin and satisfying the requirements under (1a) and (1b). If $w \in \mathcal{W}_{q a}$ is quasi-analytic with respect to the basis $\left\{v_{1}, \ldots, v_{n}\right\}$ of $\mathbb{R}^{n}$, then there exists $\widetilde{w} \in \mathcal{W}_{q a}$ of class $C^{\infty}$, satisfying the requirements under (1a) and (1b), again quasi-analytic with respect to $\left\{v_{1}, \ldots, v_{n}\right\}$ and such that $\widetilde{w}(x)=\widetilde{w}(-x)$ for all $x \in \mathbb{R}^{n}$.

Remark 3.23. It is important to note that for all weights $w$ of the various types that we have distinguished, there exists a majorant $\widetilde{w}$ of the same type and with the properties as in the first part.

Proof. The non-trivial statement in the first part are clear in view of Proposition 3.21 and its proof. As to the second part, if $w \in \mathcal{W}_{d}(0 \leq d<\infty)$ or $w \in \mathcal{W}_{\text {hol }}$ one can take $\widetilde{w}(x)=\sup _{\|y\|=\|x\|} w(y)+\exp \left(-\|x\|^{2}\right)$. The case $w \in \mathcal{W}$ is trivial. Consider the case $w \in \mathcal{W}_{\infty}$. If $\operatorname{supp} w$ is bounded then $C \exp \left(-\sqrt{\|x\|^{2}+1}\right)$ for some sufficiently large $C$ obviously suffices. Put

$$
w^{\prime}(x)=2 \inf _{m=0,1,2, \ldots} \frac{\|\| y\left\|^{m} \cdot w(y)\right\|_{\infty}}{\|x\|^{m}} \quad\left(x \in \mathbb{R}^{n}\right) .
$$

Since $\left\{\|\| y\left\|^{m} \cdot w(y)\right\|_{\infty}\right\}_{m=0}^{\infty}$ is a strictly positive logarithmically convex sequence, the analogues of (3.3) and the discussion of (3.4) apply, showing that $w^{\prime}$ can be modified into a majorant with all the required properties. Finally, the third part of Theorem 3.16 handles the quasi-analytic case.

\section{Density IN $\mathcal{O}_{w}$}

The principal aim of this section is the proof of the density of various common subspaces of the spaces $\mathcal{O}_{w}$, as dependent on the properties of the weight $w$. The principal tool is the Fourier transform on $\mathcal{O}_{w}^{\prime}$. This transform can be defined since, as it turns out, $\mathcal{O}_{w}^{\prime}$ is canonically embedded in $\mathcal{S}^{\prime}$ for any weight $w$. If $w$ is in one of the various classes of weights as in Section 3, then there is a corresponding minimal degree of regularity of the elements of $\mathcal{F}\left(\mathcal{O}_{w}^{\prime}\right)$, dependent of the particular class. In the chain of inclusions in Proposition 3.7 this regularity increases from right to left. To be more precise: if $w \in \mathcal{W}_{d}(0 \leq d \leq \infty)$ then the Fourier transforms are in 
fact functions and we show that these functions are of class $C^{[d]}$. If $w \in \mathcal{W}_{q a}$ they have the quasi-analytic property. If $w \in \mathcal{W}_{h o l}$ the transforms are holomorphic on a tubular neighbourhood of $\mathbb{R}^{n}$.

The notion of admissible spaces for a weight $w$, as motivated in Section 1, is introduced in Definition 4.11. Once the regularity properties for the elements of $\mathcal{F}\left(\mathcal{O}_{w}^{\prime}\right)$ have been established (Theorem 4.9), the density of these admissible spaces in $\mathcal{O}_{w}$ is immediate (Theorem 4.13).

The section concludes with a necessary condition for $\mathcal{P}$ to be dense in $\mathcal{O}_{w}$ for an arbitrary rapidly decreasing weight $w$.

4.1. Fourier transform on $\mathcal{O}_{w}^{\prime}$. The main result in this section is Theorem 4.13 , describing the regularity of Fourier transforms of elements of $\mathcal{O}_{w}^{\prime}$ when $w \in \mathcal{W}_{0}$. We need some preparation.

A basic fact for the spaces $\mathcal{O}_{w}$ is the density of $\mathcal{D}$. This result is due to Zapata 33 in a slightly more general context to which we return in Section 6. We include the proof, since the result is vital for the main results in this section.

Proposition 4.1. Let $w$ be a weight. Then $\mathcal{D}$ is dense in $\mathcal{O}_{w}$.

Proof (Zapata). Choose $\theta \in C_{c}^{\infty}\left(\mathbb{R}^{n}\right)$ such that $0 \leq \theta \leq 1$ and $\theta(x)=1$ for $\|x\| \leq 1$. For $h>0$, define $\theta_{h} \in C_{c}^{\infty}\left(\mathbb{R}^{n}\right)$ by $\theta_{h}(x)=\theta(h x)$; note that for all $\mu \in \mathbb{N}^{n}$ there is a constant $C_{\mu} \geq 0$ such that for all $h>0$ :

$$
\left\|\partial^{\mu} \theta_{h}\right\|_{\infty} \leq C_{\mu} h^{|\mu|} .
$$

We claim that $\lim _{h \downarrow 0} \theta_{h} f=f$ for all $f \in \mathcal{O}_{w}$, which clearly proves the proposition. In order to validate this claim, fix $f \in \mathcal{O}_{w}$ and $\alpha \in \mathbb{N}^{n}$. Let $\epsilon>0$ be given. By definition there exists $R>0$ such that

$$
\sup _{\|x\| \geq R}\left|\partial^{\alpha} f(x) \cdot w(x)\right|<\epsilon / 2 .
$$

Recalling the definition of the seminorm $p_{\alpha}$, we see that there exists a finite set of positive integers $c_{\mu}$ such that

$$
p_{\alpha}\left(f-\theta_{h} f\right) \leq\left\|\left(1-\theta_{h}\right) \partial^{\alpha} f \cdot w\right\|_{\infty}+\sum_{\mu \in \mathbb{N}^{n}, \mu \neq 0} c_{\mu}\left\|\partial^{\mu} \theta_{h} \cdot \partial^{\alpha-\mu} f \cdot w\right\|_{\infty} .
$$

For $0<h<R^{-1}$ we have $\left\|\left(1-\theta_{h}\right) \partial^{\alpha} f \cdot w\right\|_{\infty}<\epsilon / 2$, implying that for such $h$

$$
p_{\alpha}\left(f-\theta_{h} f\right)<\epsilon / 2+\sum_{\mu \in \mathbb{N}^{n}, \mu \neq 0} c_{\mu} C_{\mu} h^{|\mu|} p_{\alpha-\mu}(f) .
$$

The claim follows by taking $h \neq 0$ small enough.

If $w$ is a weight, then the injection $\mathcal{S} \hookrightarrow \mathcal{O}_{w}$ is continuous. Since $\mathcal{S}$ is evidently dense in $\mathcal{O}_{w}$ by the previous proposition, $\mathcal{O}_{w}^{\prime}$ is canonically identified with a subspace of $\mathcal{S}^{\prime}$. We use the same notation for $T \in \mathcal{O}_{w}^{\prime}$ and its image in $\mathcal{S}^{\prime}$.

Definition 4.2. If $T \in \mathcal{O}_{w}^{\prime}$, there exist a constant $C$, a non-negative integer $L$ and a finite set $\left\{\beta_{1}, \ldots, \beta_{L}\right\}$ of multi-indices such that for all $f \in \mathcal{O}_{w}$ :

$$
|\langle T, f\rangle| \leq C \max _{k=1, \ldots, L} p_{\beta_{k}}(f) .
$$

The minimum of $\max _{k=1, \ldots, L}\left|\beta_{k}\right|$, computed over all sets of multi-indices for which there exists a $C$ such that (4.1) holds for all $f \in \mathcal{O}_{w}$, is the order of $T$ in $\mathcal{O}_{w}^{\prime}$, denoted $\operatorname{ord}_{\mathcal{O}_{w}} T$. 
Remark 4.3. If $T \in \mathcal{O}_{w}^{\prime}$ then $T$ has also a finite order $\operatorname{ord}_{\mathcal{D}} T$ as a tempered distribution. It is immediate from the definitions that $\operatorname{ord}_{\mathcal{D}} T \leq \operatorname{ord}_{\mathcal{O}_{w}} T$. Inequality can occur; we give an example of this in one dimension for the constant weight 1 . Let $f(x)=x^{-2} \sin x^{3}(x \in \mathbb{R})$. Then $f \in C^{\infty}(\mathbb{R}), f \in L_{1}(\mathbb{R}, d x)$ and $f^{\prime} \notin L_{1}(\mathbb{R}, d x)$. Consider the tempered distribution $T_{f^{\prime}}$ of order zero determined by $f^{\prime}$. Since $\left|\left\langle T_{f^{\prime}}, \psi\right\rangle\right| \leq\|f\|_{1}\left\|\psi^{\prime} \cdot 1\right\|_{\infty}$ for $\psi \in \mathcal{S}, T_{f^{\prime}}$ is continuous on $\mathcal{S}$ in the topology of $\mathcal{O}_{1}$. Let $\widetilde{T}_{f^{\prime}}$ denote the unique continuous extension of $T_{f^{\prime}}$ to $\mathcal{O}_{1}$. Then $\operatorname{ord}_{\mathcal{D}} \widetilde{T}_{f^{\prime}}=0$ and from the density of $\mathcal{S}$ in $\mathcal{O}_{w}$ one concludes that ord $\mathcal{O}_{1} \widetilde{T}_{f^{\prime}} \leq 1$. Using that $f^{\prime} \notin L_{1}(\mathbb{R}, d x)$ one sees that ord $\mathcal{O}_{1} \widetilde{T}_{f^{\prime}} \neq 0$ and that therefore ord $\mathcal{O}_{1} \widetilde{T}_{f^{\prime}}=1$.

The results for the Fourier transform on $\mathcal{O}_{w}^{\prime}$ follow mainly from the degree of regularity of the map $\lambda \mapsto e_{i \lambda}$, which assumes values in $\mathcal{O}_{w}$ and is defined on a suitable domain. We need the following definitions.

Definition 4.4. Let $w$ be a weight.

1. If $w \notin \mathcal{W}_{h o l}$, put $\mathbb{C}_{w}^{n}=\mathbb{R}^{n}$. If $w \in \mathcal{W}_{\text {hol }}$, put $\mathbb{C}_{w}^{n}=\left\{\lambda \in \mathbb{C}^{n} \mid\|\operatorname{Im} \lambda\|<\tau_{w}\right\}$ where $\tau_{w}$ is as in Section 3 .

2. If $d$ is a non-negative integer, $P \in \mathcal{P}_{d}$ and $w \in \mathcal{W}_{d}$, define $\Psi_{P}: \mathbb{C}_{w}^{n} \mapsto \mathcal{O}_{w}$ by $\Psi_{P}(\lambda)=P e_{i \lambda}\left(\lambda \in \mathbb{C}_{w}^{n}\right)$.

The notation $\mathbb{C}_{w}^{n}$ for the domains has been chosen in order to treat the holomorphic and the non-holomorphic case in a uniform formulation as much as possible.

Our next three results concern regularity of the maps $\Psi_{P}$.

Proposition 4.5. Let $0 \leq d<\infty$ be an integer. If $P \in \mathcal{P}_{d}$ and $w \in \mathcal{W}_{d}$, then $\Psi_{P}: \mathbb{C}_{w}^{n} \mapsto \mathcal{O}_{w}$ is continuous.

Proof. We treat the case where $w \notin \mathcal{W}_{\text {hol }}$; the case where $w \in \mathcal{W}_{\text {hol }}$ is similar. We then have to show that

$$
\lim _{\|h\| \rightarrow 0} p_{\alpha}\left(\Psi_{P}(\lambda+h)-\Psi_{P}(\lambda)\right)=0
$$

for all fixed $\lambda \in \mathbb{R}^{n}$ and $\alpha \in \mathbb{N}^{n}$. The definitions make it obvious that

$$
p_{\alpha}\left(\Psi_{P}(\lambda+h)-\Psi_{P}(\lambda)\right) \leq \sum_{\mu+\nu=\alpha} c_{\mu, \nu}\left\|\partial_{x}^{\mu} P \cdot \partial_{x}^{\nu}\left(e_{i(\lambda+h)}-e_{i \lambda}\right) \cdot w\right\|_{\infty}
$$

for some non-negative integers $c_{\mu, \nu}$. By a moment's thought, it is therefore sufficient to show that

$$
\lim _{\|h\| \rightarrow 0}\left\|(1+\|x\|)^{d}\left\{(\lambda+h)^{\nu} e_{i h}-\lambda^{\nu}\right\} \cdot w\right\|_{\infty}=0
$$

for all $\nu$ occurring in the summation. This holds in fact for all $\nu \in \mathbb{N}^{n}$. To see this, in view of

$$
\begin{aligned}
& \left\|(1+\|x\|)^{d}\left\{(\lambda+h)^{\nu} e_{i h}-\lambda^{\nu}\right\} \cdot w\right\|_{\infty} \leq \\
& \leq\left\|(1+\|x\|)^{d}\left\{(\lambda+h)^{\nu} e_{i h}-\lambda^{\nu} e_{i h}\right\} \cdot w\right\|_{\infty}+\left\|(1+\|x\|)^{d}\left\{\lambda^{\nu} e_{i h}-\lambda^{\nu}\right\} \cdot w\right\|_{\infty} \\
& =\left|(\lambda+h)^{\nu}-\lambda^{\nu}\right|\left\|(1+\|x\|)^{d} \cdot w\right\|_{\infty}+|\lambda|^{\nu}\left\|(1+\|x\|)^{d}\left\{e_{i h}-1\right\} \cdot w\right\|_{\infty},
\end{aligned}
$$

it is sufficient to prove that

$$
\lim _{\|h\| \rightarrow 0}\left\|(1+\|x\|)^{d}\left\{e_{i h}-1\right\} \cdot w(x)\right\|_{\infty}=0 .
$$

Let $\epsilon>0$. Choose $R>0$ such that $\sup _{\|x\| \geq R}\left|(1+\|x\|)^{d}\left\{e_{i h}(x)-1\right\} \cdot w(x)\right|<\epsilon$ for all $h$, which is possible since $w \in \mathcal{W}_{d}$. In addition, since $|\exp (i t)-1| \leq|t|$ 
for $t \in \mathbb{R}$, we can arrange that also $\sup _{\|x\|<R}\left|(1+\|x\|)^{d}\left\{e_{i h}(x)-1\right\} \cdot w(x)\right|<\epsilon$ by taking $\|h\|$ small enough. This proves (4.2) and completes the proof when $w \notin \mathcal{W}_{\text {hol }}$.

Proposition 4.6. Let $0 \leq d<\infty$ be an integer. If $P \in \mathcal{P}_{d}$ and $w \in \mathcal{W}_{d+1}$, then $\frac{\partial \Psi_{P}}{\partial \lambda_{j}}$ exists for $j=1, \ldots, n$ on $\mathbb{C}_{w}^{n}$ in the topology of $\mathcal{O}_{w}$ and is given by

$$
\left(\frac{\partial \Psi_{P}}{\partial \lambda_{j}}(\lambda)\right)(x)=i x_{j} P(x) e^{i(\lambda, x)} \quad\left(\lambda \in \mathbb{C}_{w}^{n}, x \in \mathbb{R}^{n}\right) .
$$

If $w \in \mathcal{W}_{\text {hol }}$, the derivatives are to be interpreted as complex derivatives.

Proof. We treat the case where $w \notin \mathcal{W}_{\text {hol }}$; the case where $w \in \mathcal{W}_{\text {hol }}$ is similar. Fix $1 \leq j \leq n$. The definitions imply we then have to prove that

$$
\lim _{h \rightarrow 0} p_{\alpha}\left(U V_{h}\right)=0
$$

for all fixed $\alpha \in \mathbb{N}^{n}$ and $\lambda \in \mathbb{R}^{n}$, where $U$ and $V_{h}(0 \neq h \in \mathbb{R})$ in $C^{\infty}\left(\mathbb{R}^{n}\right)$ are defined by

$$
\begin{aligned}
U(x) & =P(x) e^{i(\lambda, x)} & & \left(x \in \mathbb{R}^{n}\right) \\
V_{h}(x) & =i x_{j}-\frac{e^{i h x_{j}}-1}{h} & & \left(x \in \mathbb{R}^{n}\right) .
\end{aligned}
$$

The definition of $p_{\alpha}$ implies that is sufficient to prove

$$
\lim _{h \rightarrow 0}\left\|(1+\|x\|)^{d} \partial_{x}^{\nu} V_{h} \cdot w\right\|_{\infty}=0
$$

for all $\nu \in \mathbb{N}^{n}$. For this, we distinguish three cases:

1. Assume $|\nu|=0$. Let $\epsilon>0$ be given. From $\mid \exp (i t)-1)|\leq| t \mid(t \in \mathbb{R})$ we see that $\left|V_{h}(x)\right| \leq 2\|x\|$ for all $h \neq 0$ and all $x$. Since $w \in \mathcal{W}_{d+1}$, we can therefore select $R>0$ such that $\sup _{\|x\| \geq R}\left|(1+\|x\|)^{d} V_{h}(x) \cdot w(x)\right|<\epsilon$ for all $h \neq 0$. Next, since $|\exp (i t)-1-i t| \leq t^{2} / 2$ for $t \in \mathbb{R}$ it follows easily that we can then arrange that $\sup _{\|x\|<R}\left|(1+\|x\|)^{d} V_{h}(x) \cdot w(x)\right|<\epsilon$ as well, by taking $\|h\|$ small enough. This proves (4.4) in case $|\nu|=0$.

2. Assume $|\nu|=1$. Then the only possibility for $\partial_{x}^{\nu}$ which does not lead to the trivial situation $\partial_{x}^{\nu} V_{h}=0$, is $\partial_{x}^{\nu}=\left(\partial / \partial x_{j}\right)$, in which case one verifies that for $h \neq 0$ :

$$
\left\|(1+\|x\|)^{d} \partial_{x}^{\nu} V_{h} \cdot w\right\|_{\infty} \leq|h|\left\|(1+\|x\|)^{d}\right\| x\|\cdot w\|_{\infty} .
$$

This proves (4.4) for $|\nu|=1$.

3. Assume $|\nu| \geq 2$. As in the previous case, the only possibility for $\partial_{x}^{\nu}$ which does not lead to the trivial situation $\partial_{x}^{\nu} V_{h}=0$, is $\partial_{x}^{\nu}=\left(\partial / \partial x_{j}\right)^{|\nu|}$, in which case one verifies that for $h \neq 0$ :

$$
\left\|(1+\|x\|)^{d} \partial_{x}^{\nu} V_{h} \cdot w\right\|_{\infty} \leq|h|^{|\nu|-1}\left\|(1+\|x\|)^{d} \cdot w\right\|_{\infty} .
$$

This proves (4.4) for $|\nu| \geq 2$.

This concludes the proof for $w \notin \mathcal{W}_{\text {hol }}$.

The following corollary follows by induction, using Proposition 4.5 and Proposition 4.6. 
Corollary 4.7. Let $d \geq 0$ be an integer. If $w \in \mathcal{W}_{d}$, then $\Psi_{1}: \mathbb{C}_{w}^{n} \mapsto \mathcal{O}_{w}$ is strongly of class $C^{d}$, with derivatives given by

$$
\left(\partial^{\alpha} \Psi_{1}(\lambda)\right)(x)=i^{|\alpha|} x^{\alpha} e_{i \lambda}(x) \quad\left(\lambda \in \mathbb{C}_{w}^{n}, x \in \mathbb{R}^{n} ; \alpha \in \mathbb{N}^{n},|\alpha| \leq d\right) .
$$

If $w \in \mathcal{W}_{\text {hol }}$, then the derivatives exist for all $\alpha \in \mathbb{N}^{n}$ and are to be interpreted as complex derivatives.

As a last auxiliary result, we need the following estimates.

Lemma 4.8. Let $\alpha \in \mathbb{N}^{n}$. If $w \in \mathcal{W}_{|\alpha|}$, then for all $\beta \in \mathbb{N}^{n}$ and $\lambda \in \mathbb{C}_{w}^{n}$ :

$p_{\beta}\left(x^{\alpha} e^{i(\lambda, x)}\right) \leq 2^{|\beta|}(1+\|\lambda\|)^{|\beta|} \prod_{j=1}^{n}\left(1+\alpha_{j}\right)^{|\beta|} \cdot\left\|\prod_{j=1}^{n}\left(1+\left|x_{j}\right|^{\alpha_{j}}\right) \cdot e^{-(\operatorname{Im} \lambda, x)} \cdot w(x)\right\|_{\infty}$.

Proof. It is evident that

$$
p_{\beta}\left(x^{\alpha} e^{i(\lambda, x)}\right) \leq \sum_{\mu+\nu=\beta} c_{\mu, \nu}\left\|\partial_{x}^{\mu} x^{\alpha} \cdot \partial_{x}^{\nu} e^{i(\lambda, x)} \cdot w(x)\right\|_{\infty}
$$

for some non-negative integers $c_{\mu, \nu}$ satisfying

$$
\sum_{\mu+\nu=\beta} c_{\mu, \nu}=2^{|\beta|} .
$$

We estimate the functions occurring in the summands in (4.5). First, in such a summand one has for all $x$ :

$$
\begin{aligned}
\left|\partial_{x}^{\mu} x^{\alpha}\right| & \leq \prod_{j=1}^{n}\left(1+\alpha_{j}\right)^{\beta_{j}}\left(1+\left|x_{j}\right|^{\alpha_{j}}\right) \\
& \leq \prod_{j=1}^{n}\left(1+\alpha_{j}\right)^{|\beta|}\left(1+\left|x_{j}\right|^{\alpha_{j}}\right) .
\end{aligned}
$$

In addition, in such a summand one has for all $x$ and $\lambda$ :

$$
\begin{aligned}
\left|\partial_{x}^{\nu} e^{i(\lambda, x)}\right| & \leq \prod_{j=1}^{n}\left(1+\left|\lambda_{j}\right|\right)^{\beta_{j}} \cdot e^{-(\operatorname{Im} \lambda, x)} \\
& \leq(1+\|\lambda\|)^{|\beta|} e^{-(\operatorname{Im} \lambda, x)} .
\end{aligned}
$$

The statement now follows from an application of the estimates in (4.5) and a subsequent use of (4.6).

We are now in the position to prove the basic result for the Fourier transform on $\mathcal{O}_{w}^{\prime}$.

Theorem 4.9. Let $d \in\{0,1,2, \ldots, \infty\}$, assume $w \in \mathcal{W}_{d}$ and suppose $T \in \mathcal{O}_{w}^{\prime}$. Put $N=\operatorname{ord}_{\mathcal{O}_{w}^{\prime}} T$. Then:

1. There is a natural injective map $\mathcal{O}_{w}^{\prime} \rightarrow \mathcal{S}^{\prime}$, defined by letting to $T \in \mathcal{O}_{w}^{\prime}$ correspond the tempered distribution $f \mapsto\langle T, f\rangle(f \in \mathcal{S})$, which we again denote by $T$. The order of $T$ as a tempered distribution is at most $N$. 
2. The Fourier transform of $T$, where $T$ is viewed as a tempered distribution, is a continuous function $\widehat{T}$. It is given for $\lambda \in \mathbb{R}^{n}$ by

$$
\widehat{T}(\lambda)=(2 \pi)^{-n / 2}\left\langle T, e_{-i \lambda}\right\rangle .
$$

The map from $\mathcal{O}_{w}^{\prime}$ into $C\left(\mathbb{R}^{n}\right)$ defined by $T \mapsto \widehat{T}$ is injective.

3. $\widehat{T}$ is of class $C^{d}$ on $\mathbb{R}^{n}$, with derivatives for $\lambda \in \mathbb{R}^{n}$ and $\alpha \in \mathbb{N}^{n}(|\alpha| \leq d)$ given by

$$
\partial^{\alpha} \widehat{T}(\lambda)=(2 \pi)^{-n / 2}(-i)^{|\alpha|}\left\langle T, x^{\alpha} e_{-i \lambda}\right\rangle .
$$

4. There exists a constant $C>0$ such that for all $\lambda \in \mathbb{R}^{n}$ and for all $\alpha \in \mathbb{N}^{n}$ with $|\alpha| \leq d$ :

$$
\left|\partial^{\alpha} \widehat{T}(\lambda)\right| \leq C(1+\|\lambda\|)^{N} \prod_{j=1}^{n}\left(1+\alpha_{j}\right)^{N}\left\|\prod_{j=1}^{n}\left(1+\left|x_{j}\right|^{\alpha_{j}}\right) \cdot w(x)\right\|_{\infty} .
$$

5. If $w \in \mathcal{W}_{\text {hol }}$, then (4.7) defines a holomorphic extension of $\widehat{T}$ to $\mathbb{C}_{w}^{n}$, also denoted by $\widehat{T}$. The complex derivatives of $\widehat{T}$ on $\mathbb{C}_{w}^{n}$ are then given by (4.8) and there exists a constant $C>0$ such that for all $\lambda \in \mathbb{C}_{w}^{n}$ and all $\alpha \in \mathbb{N}^{n}$ :

$$
\left|\partial^{\alpha} \widehat{T}(\lambda)\right| \leq C(1+\|\lambda\|)^{N} \prod_{j=1}^{n}\left(1+\alpha_{j}\right)^{N}\left\|\prod_{j=1}^{n}\left(1+\left|x_{j}\right|^{\alpha_{j}}\right) \cdot e^{(\operatorname{Im} \lambda, x)} \cdot w(x)\right\|_{\infty}
$$

6. If $w$ is standard quasi-analytic, then there exist a constant $C>0$ and nonnegative sequences $\{\widetilde{M}(j, m)\}_{m=0}^{\infty}(j=1, \ldots, n)$ such that

$$
\sum_{m=1}^{\infty} \frac{1}{\sqrt[m]{\widetilde{M}(j, m)}}=\infty \quad(j=1, \ldots, n)
$$

and, for all $\lambda \in \mathbb{R}^{n}$ and all $\alpha \in \mathbb{N}^{n}$ :

$$
\left|\partial^{\alpha} \widehat{T}(\lambda)\right| \leq C(1+\|\lambda\|)^{N} \prod_{j=1}^{n} \widetilde{M}\left(j, \alpha_{j}\right) .
$$

Moreover, if $w$ is standard quasi-analytic and if there exists $\lambda_{0} \in \mathbb{R}^{n}$ such that $\partial^{\alpha} \widehat{T}\left(\lambda_{0}\right)=0$ for all $\alpha \in \mathbb{N}^{n}$, then $T=0$.

Proof. As to part 1: this was already observed following Proposition 4.1.

For part 2, we first consider as preparation a weight $\widetilde{w} \in \mathcal{W}_{0}$ which is separated from zero on compact sets. Let $f \in \mathcal{D}$ be arbitrary. The map $\lambda \mapsto f(\lambda) e_{-i \lambda}=$ $f(\lambda) \Psi_{1}(-\lambda)$ is then continuous by Proposition 1.5, evidently compactly supported and with values in $\mathcal{O}_{\widetilde{w}}$, which is a Fréchet space by Proposition 3.21. Therefore, as a consequence of [27, Thm. 3.27], the weak integral

$$
(2 \pi)^{-n / 2} \int_{\mathbb{R}^{n}} f(\lambda) e_{-i \lambda} d \lambda
$$

exists in $\mathcal{O}_{\widetilde{w}}$. Applying point evaluations, which are continuous since $\widetilde{w}$ has no zeros, the integral is identified as $\widehat{f}$. We now turn to the statements in part 2 and note that the function $\widehat{T}$ as described in the theorem is certainly continuous on $\mathbb{R}^{n}$ (in fact on $\mathbb{C}_{w}^{n}$ ) as a consequence of Proposition 4.5. We also note that, since $w \in \mathcal{W}_{d} \subset \mathcal{W}_{0}$, Theorem 3.22 allows us to choose a majorant $\widetilde{w} \in \mathcal{W}_{0}$ of $w$ which 
is separated from zero on compact sets. The previous result on the weak integrals in $\mathcal{O}_{\widetilde{w}}$ then imply, in view of the continuity of the injection $\mathcal{O}_{\widetilde{w}} \hookrightarrow \mathcal{O}_{w}$, that

$$
\langle T, \widehat{f}\rangle=(2 \pi)^{-n / 2} \int_{\mathbb{R}^{n}} f(\lambda)\left\langle T, e_{-i \lambda}\right\rangle d \lambda
$$

for all $f \in \mathcal{D}$. Therefore, with $\mathcal{F}(T)$ denoting the Fourier transform of the tempered distribution $T$, we have

$$
\langle\mathcal{F}(T), f\rangle=\int_{\mathbb{R}^{n}} f(\lambda) \widehat{T}(\lambda) d \lambda
$$

for all $f \in \mathcal{D}$. Now note that as a consequence of (4.1) and the estimates in Lemma 4.8 (applied with $\alpha=0$ ), $\widehat{T}$ is of at most polynomial growth on $\mathbb{R}^{n}$. In view of the density of $\mathcal{D}$ in $\mathcal{S}$ and the continuity of $\mathcal{F}(T)$, the dominated convergence theorem implies that (4.9) actually holds for all $f \in \mathcal{S}$, showing that the function $\widehat{T}$ represents the Fourier transform of the tempered distribution $T$ as claimed. If $\widehat{T}=0$ then $T=0$ in $\mathcal{S}^{\prime}$, implying $T=0$ in $\mathcal{O}_{w}^{\prime}$ in view of part 1 . This concludes the proof of part 2 .

Corollary 4.7 implies the degree of differentiability and the expressions for the derivatives in part 3 and part 5.

In view of (4.1), part 4 and the estimates in part 5 follow immediately from Lemma 4.8.

We now prove the assertions on the sequences in part 6 as a consequence of the estimates in part 4 , as follows. For $j=1, \ldots, n$ and $m \geq 0$ put

$$
\widetilde{M}(j, m)=(1+m)^{N}\left\|\left(1+\left|x_{j}\right|^{m}\right) \cdot w^{1 / n}(x)\right\|_{\infty} .
$$

Since $w^{1 / n}$ is a standard quasi-analytic weight by Lemma 3.11, we have

$$
\sum_{m=1}^{\infty}\left\|x_{j}^{m} \cdot w^{1 / n}\right\|_{\infty}^{-1 / m}=\infty \quad(j=1, \ldots, n) .
$$

Fix $j$. If there does not exists $x \in \mathbb{R}^{n}$ with $\left|x_{j}\right|>1$ such that $w(x)>0$, then certainly

$$
\sum_{m=1}^{\infty} \frac{1}{\sqrt[m]{\widetilde{M}(j, m)}}=\infty \quad(j=1, \ldots, n)
$$

since the terms tend to a constant. In the remaining case where there exists $x \in \mathbb{R}^{n}$ with $\left|x_{j}\right|>1$ such that $w(x)>0$, one verifies that the quotient of the terms in the series in (4.10) and (4.11) tends to 1 as $m \rightarrow \infty$; (4.11) therefore follows from (4.10).

Finally, suppose that all derivatives of $\widehat{T}$ vanish at some $\lambda_{0}$. Then it follows from the estimates on the derivatives and Theorem B.1 on quasi-analytic classes that the translate of $\widehat{T}$ over $\lambda_{0}$ is identically zero. Therefore $\widehat{T}=0$, implying $T=0$ by part 2.

Remark 4.10. Part 5 in the theorem, when specialized to $\alpha=0$, is one half of a Paley-Wiener type theorem. For a distribution $T$ with compact convex support $K$ one can in fact derive the easy half of the geometric Paley-Wiener theorem (cf. [30, Exerc. 29.5]) from this specialization of part 5, since such $T$ is continuous on $\mathcal{D}$ in the induced topology of $\mathcal{O}_{1_{K}}$ by [29, p. 99, Theorem XXXIV.1]. 
4.2. Admissible spaces and their density in $\mathcal{O}_{w}$. It is now easy to derive various density results from Theorem 4.9. As may have become apparent from Section 1, in applications the relevant property of a dense subspace of $\mathcal{O}_{w}$ is not the density itself, but the property that it has the same closure as any other dense subspace of $\mathcal{O}_{w}$, and $\mathcal{D}$ in particular. This leads to the concept of admissible spaces for weights (Definition 4.11) and motivates the formulation of Theorem 4.13.

Definition 4.11. Let $w$ be a weight. An admissible space for $w$ is any of the following subspaces of $C^{\infty}\left(\mathbb{R}^{n}\right)$ :

1. If $w \notin \mathcal{W}_{0}$ : a dense subspace of $\mathcal{S}$.

2. If $w \in \mathcal{W}_{0}$ but $w \notin \mathcal{W}_{q a}$ :

(a) a dense subspace of $\mathcal{S}$, or

(b) a subspace of the form $\operatorname{Span}\left\{e_{i \lambda} \mid \lambda \in E\right\}$, where $E \subset \mathbb{R}^{n}$ is dense.

3. If $w \in \mathcal{W}_{\text {qa }}$ but $w \notin \mathcal{W}_{\text {hol }}$ :

(a) a dense subspace of $\mathcal{S}$, or

(b) a subspace of the form $\operatorname{Span}\left\{e_{i \lambda} \mid \lambda \in E\right\}$, where $E \subset \mathbb{R}^{n}$ is such that $\bar{E}$ has non-empty interior, or

(c) $\mathcal{P}$.

4. If $w \in \mathcal{W}_{\text {hol }}$ :

(a) a dense subspace of $\mathcal{S}$, or

(b) a subspace of the form $\operatorname{Span}\left\{e_{i \lambda} \mid \lambda \in E\right\}$, where $E \subset \mathbb{C}_{w}^{n}$ is such that any holomorphic function on $\mathbb{C}_{w}^{n}$ vanishing on $E$ is identically zero on $\mathbb{C}_{w}^{n}$, or

(c) $\mathcal{P}$.

Remark 4.12. In the one-dimensional case, the possible choices for spectral subsets $E$ as required above for $w \in \mathcal{W}_{h o l}$ can alternatively be described as the subsets of $\mathbb{C}_{w}$ having an accumulation point in $\mathbb{C}_{w}$. In arbitrary dimension, the situation is more involved and a simple description of such uniqueness sets is not known. $\mathbb{R}^{n}$ is a uniqueness set for holomorphic functions on $\mathbb{C}^{n}$ and furthermore the boundary uniqueness theorem and the maximum principle [7, p. 289] supply non-trivial examples of uniqueness sets for holomorphic functions on $\mathbb{C}_{w}^{n}$. A somewhere dense subset of $\mathbb{R}^{n}$ is a uniqueness set for holomorphic functions on $\mathbb{C}_{w}^{n}$.

Theorem 4.13. Let $w$ be a weight. Then all admissible spaces for $w$ are subspaces of $\mathcal{O}_{w}$ and they all have the same closure in $\mathcal{O}_{w}$. In fact, they are all dense.

Proof. It is obvious that the admissible spaces are subspaces. To prove that they are all dense, we start with an arbitrary weight such that $w \notin \mathcal{W}_{0}$ and then prove the various incremental statements on density as the properties of $w$ improve.

In the starting case, the density in $\mathcal{O}_{w}$ of an arbitrary dense subspace of $\mathcal{S}$ follows from Lemma 1.1. the density of $\mathcal{S}$ in $\mathcal{O}_{w}$ and the continuity of the injection $\mathcal{S} \hookrightarrow \mathcal{O}_{w}$.

In case $w \in \mathcal{W}_{0}$ but $w \notin \mathcal{W}_{q a}$, suppose that $T \in \mathcal{O}_{w}^{\prime}$ vanishes on the span of the exponentials as described in Definition 4.11. By the continuity of $\widehat{T}$ we conclude that $\widehat{T}=0$, hence $T=0$.

In case $w \in \mathcal{W}_{q a}$ but $w \notin \mathcal{W}_{\text {hol }}$, then as a consequence of Proposition 3.7 we may assume that $w$ is standard quasi-analytic. In that case, if $T \in \mathcal{O}_{w}^{\prime}$ vanishes on the span of the exponentials as described in Definition 4.11, then by continuity there exists an open set on which $\widehat{T}$ vanishes. In view of part 6 in Theorem 4.9 we 
conclude that $T=0$. Also, if $T \in \mathcal{O}_{w}^{\prime}$ vanishes on $\mathcal{P}$, then part 3 and part 6 of Theorem 4.9 show that $T=0$.

Finally, in case $w \in \mathcal{W}_{\text {hol }}$ the remaining statement on the density of the span of the exponentials as described follows from the holomorphy of $\widehat{T}$ on $\mathbb{C}_{w}^{n}$ and again the injectivity of the map $T \mapsto \widehat{T}$.

Remark 4.14. 1. A crucial step in the proof of proof of Theorem 4.13 is the conclusion that $T$ vanishes on $\mathcal{O}_{w}$ whenever the function $\widehat{T}$-which could as well have been defined directly as $\widehat{T}=\left\langle T, e_{-i \lambda}\right\rangle$-vanishes on $\mathbb{R}^{n}$. For this step it is not essential that $\widehat{T}$ can be interpreted as a Fourier transform of a tempered distribution. Indeed, the conclusion follows by considering a weak integral $\int_{\mathbb{R}^{n}} f(\lambda) e_{-i \lambda} d \lambda$ in $\mathcal{O}_{w}$ as in the proof of Theorem 4.9 when one uses that $\mathcal{F}(\mathcal{D})$ is dense in $\mathcal{S}$ and therefore also in $\mathcal{O}_{w}$. A range results of this kind appears to be essential; the closure theorems on exponentials and polynomials in $\mathcal{O}_{w}$ to considerable extent ultimately rest on harmonic analysis. This connection with harmonic analysis will become even more apparent in the discussion of possible generalizations in Section 7 .

2. It is instructive to note that the various density results in $\mathcal{O}_{w}$ have different origins. The density of $\mathcal{D}$ and $\mathcal{S}$ for general $w$ can be proved in a direct fashion. The density of the trigonometric functions for $w \in \mathcal{W}_{0}$ has, as noted above, its origin in harmonic analysis. For $w \in \mathcal{W}_{\text {hol }}$ this can still be said for the additional density of $\mathcal{P}$ and the span of exponentials as described. For $w \in \mathcal{W}_{q a}$ but $w \notin \mathcal{W}_{\text {hol }}$ both the density of $\mathcal{P}$ and the density of the span of exponentials as described are relatively deep statements, based not only on a result from harmonic analysis but on a theorem on quasi-analytic classes in several variables as well.

3. The theorem can be generalized (cf. Theorem 6.19).

For $w \in \mathcal{W}_{\infty}$ we have established that the condition $w \in \mathcal{W}_{q a}$ is sufficient for the polynomials to be dense in $\mathcal{O}_{w}$. The following proposition, which should be compared with Corollary 3.18, gives a necessary condition. It concludes our study of density in $\mathcal{O}_{w}$.

Proposition 4.15. Let $w \in \mathcal{W}_{\infty}$ and suppose that $\mathcal{P}$ is dense in $\mathcal{O}_{w}$. If $x, y \in \mathbb{R}^{n}$ are such that $y \neq 0$ and such that $t \mapsto w(x+t y)$ is Lebesgue measurable on $\mathbb{R}$, then

$$
\int_{-\infty}^{\infty} \frac{\log w(x+t y)}{1+t^{2}}=-\infty
$$

Proof. Fix $x$ and $y$ as in the hypotheses. Define $\iota: \mathbb{R} \mapsto \mathbb{R}^{n}$ by $\iota(t)=x+t y$. If $f$ is a function on $\mathbb{R}^{n}$ let $\iota^{*} f=f \circ \iota$ be its pull-back to $\mathbb{R}$. Then $\iota^{*} w$ is a rapidly decreasing weight on $\mathbb{R}$. Consider the one-dimensional Bernstein space $C_{\iota^{*} w}=\left\{f \in C(\mathbb{R}) \mid \lim _{|x| \rightarrow \infty} f \cdot \iota^{*} w=0\right\}$, supplied with the topology given by the seminorm $f \mapsto\left\|f \cdot \iota^{*} w\right\|_{\infty}$. Then $\iota^{*}$ maps $\mathcal{O}_{w}$ continuously into $C_{\iota^{*} w}$. Furthermore $\iota^{*} \mathcal{P}\left(\mathbb{R}^{n}\right)=\mathcal{P}(\mathbb{R})$ and $\iota^{*} \mathcal{D}\left(\mathbb{R}^{n}\right)=\mathcal{D}(\mathbb{R})$. Now if $\mathcal{P}\left(\mathbb{R}^{n}\right)$ is dense in $\mathcal{O}_{w}$, i.e. if $\mathcal{P}\left(\mathbb{R}^{n}\right)$ and $\mathcal{D}\left(\mathbb{R}^{n}\right)$ have the same closure in $\mathcal{O}_{w}$, then we conclude from this and Lemma 1.1 that $\mathcal{P}(\mathbb{R})$ and $\mathcal{D}(\mathbb{R})$ have the same closure in $C_{\iota^{*} w}$. Since $\mathcal{D}(\mathbb{R})$ is dense in $C_{\iota^{*} w}$, so is $\mathcal{P}(\mathbb{R})$. Therefore the weight $\iota^{*} w$ must satisfy Hall's integral condition [10, 22, i.e. (4.12) must hold. 


\section{Subspaces With equal Closure}

We are now able to prove the basic theorems on subspaces with equal closure. These are supplemented with a discussion of a natural partial filtration on topological vector spaces. The latter is motivated by the question what the amount of freedom is to obtain variations on (1.1), as depending on the generator, and leads to the concept of admissible spaces for elements of a topological vector space.

For ease of formulation we limit ourselves to locally convex spaces. In view of practice, this is not too severe a limitation and if necessary it is, on basis of Theorem A.2, easy to adjust the results to the situation of finitely generated subspaces in non locally convex spaces.

5.1. Formalization of the method. The following basic theorem is immediate from the combination of Theorem A.2 and Theorem 4.13. The admissible spaces for weights in its formulation can be found in Definition 4.11 .

Theorem 5.1 (Subspaces with equal closure). Let $V$ be a locally convex topological vector space and let $\mathcal{G} \subset V$ be a non-empty set of generators. Suppose that for each $g \in \mathcal{G}$ a weight $w_{g}$ on $\mathbb{R}^{n_{g}}$ is given, together with a continuous linear map from $\mathcal{O}_{w_{g}}$ into $V$ denoted by $f \mapsto f \cdot g\left(f \in \mathcal{O}_{w_{g}}\right)$. For each $g \in \mathcal{G}$ choose an admissible space $L_{g}$ for $w_{g}$ and consider $L=\sum_{g \in \mathcal{G}} L_{g} \cdot g$. Then:

1. The closure of $L$ is independent of the choice of the $L_{g}$.

2. The annihilator of $L$ is independent of the choice of the $L_{g}$ and equal to $\bigcap_{g \in \mathcal{G}}\left(L_{g}^{\prime} \cdot g\right)^{\perp}$ for any choice of admissible spaces $L_{g}^{\prime}$.

3. The sequential closure of $L$ is independent of the choice of the $L_{g}$ when $\mathcal{G}$ is finite.

In the applications we have in mind $V$ is a space of functions, equivalence classes of functions, distributions, sequences etc. with a subset of $\mathbb{R}^{n}$ as underlying point set. The $n_{g}$ are then all equal to $n$ and we will assume this for the remainder of the discussion. The linear map in the above theorem is in those situations the appropriate type of multiplication by smooth functions, as it is defined in $V$. As the reader may verify in examples, this multiplication invariably results in a natural module structure over $\mathcal{P}, \mathcal{E}_{\text {im }}$ and $\mathcal{D}$ for some - in general proper-subspaces of $V$.

The salient point in the above theorem is the possibility to switch from one choice of admissible spaces to another more convenient one (and in particular to the choice of $\mathcal{D}$ for all $g \in \mathcal{G}$ ) without affecting the closure of the subspace involved. As explained in Section $\mathbb{1}$, this is the mechanism which makes the non-trivial analysis in the spaces $\mathcal{O}_{w}$ immediately available in other situations.

We briefly summarize the switching possibilities. If $w_{g} \in \mathcal{W}$ then one can choose as admissible space $L_{g}$ a dense subspace of $\mathcal{S}$ (such as notably $\mathcal{D}$ ). If $w_{g} \in \mathcal{W}_{0}$, then in addition one can choose a space of the form $\operatorname{Span}\left\{e_{i \lambda} \mid \lambda \in E\right\}$, where $E \subset \mathbb{R}^{n}$ is dense. If $w_{g} \in \mathcal{W}_{q a}$, then there is still more choice since for $L_{g}$ one may now in addition also choose $\mathcal{P}$ or $\operatorname{Span}\left\{e_{i \lambda} \mid \lambda \in E\right\}$ if $E$ is a somewhere dense subset of $\mathbb{R}^{n}$. If $w_{g} \in \mathcal{W}_{h o l}$ then maximum choice is possible since in addition to all spaces just described there is then another type of admissible space, related to the exponentials. This additional degree of freedom depends on more details of the holomorphic weight and we refer to Definition 4.11 for the precise description.

The choices for admissible spaces can be made independently for all $g \in \mathcal{G}$ and all possible resulting subspaces $V$ have the same closure. An application of the 
theorem could in many situation e.g. be the conclusion that the modules generated by $\mathcal{G}$ over $\mathcal{D}, \mathcal{E}_{\text {im }}$ and $\mathcal{P}$ all three have the same closure, and that for a description of this closure equivalently: a description of the annihilator - one can assume that the module is generated over $\mathcal{D}$, which in most situations is a convenient choice. It is apparent that such a conclusion is a special case of the assertions in the theorem.

As an illustration we give some straightforward examples, returning to more elaborate situations in Section 6 .

Example 5.2. 1 . Let $\Omega \subset \mathbb{R}^{n}$ be open and non-empty and let $V=C_{c}^{\infty}(\Omega)^{\prime}$. Supply $C_{c}^{\infty}(\Omega)^{\prime}$ with its weak topology and consider $g=1 \in C_{c}^{\infty}(\Omega)^{\prime}$. For the holomorphic weight $w(x)=\exp \left(-\|x\|^{2}\right)$ the map $f \mapsto f \cdot g$ is continuous from $\mathcal{O}_{w}$ into $C_{c}^{\infty}(\Omega)^{\prime}$. Since $\mathcal{D} \cdot 1 \supset C_{c}^{\infty}(\Omega)$ and the latter space is well known to be sequentially dense in $C_{c}^{\infty}(\Omega)^{\prime}$, the same holds for $\mathcal{D} \cdot 1$. The third part of the theorem then asserts that this sequential density also holds for $\mathcal{P} \cdot 1$ and any space of the form $\operatorname{Span}\left\{e_{i \lambda} \cdot 1 \mid \lambda \in E\right\}$, where $E \subset \mathbb{C}^{n}$ is a uniqueness set for holomorphic functions on $\mathbb{C}^{n}$.

2. Let $V=\mathcal{S}$ and let $g(x)=\exp \left(-\|x\|^{2}\right) \in \mathcal{S}$. For the holomorphic weight $w(x)=\exp \left(-\|x\|^{2}\right)$ the map $f \mapsto f \cdot g$ is continuous from $\mathcal{O}_{w}$ into $\mathcal{S}$. Since $\mathcal{D} \cdot \exp \left(-\|x\|^{2}\right)=\mathcal{D}$ is dense in $\mathcal{S}$, we conclude from the theorem that $\mathcal{P}$. $\exp \left(-\|x\|^{2}\right)$ is also dense, as is $\operatorname{Span}\left\{e_{i \lambda} \cdot \exp \left(-\|x\|^{2}\right) \mid \lambda \in E\right\}$, where $E \subset \mathbb{C}^{n}$ is a uniqueness set for holomorphic functions on $\mathbb{C}^{n}$.

3. Let $V=L_{p}\left(\mathbb{R}^{n}, d x\right)(1 \leq p<\infty)$ and consider $g(x) \in L_{p}\left(\mathbb{R}^{n}, d x\right)$ given by $g(x)=\exp \left(-\sqrt{\|x\|^{2}+1} / \log \sqrt{\|x\|^{2}+2}\right)$. Let $w$ be a weight which is equal to $\exp (-\epsilon\|x\| / 2 \log \|x\|)$ for large $\|x\|$. Then $w$ is quasi-analytic by Proposition 3.20; the associated map $f \mapsto f \cdot g$ from $\mathcal{O}_{w}$ into $V=L_{p}\left(\mathbb{R}^{n}, d x\right)$ is then continuous. Since $\mathcal{D} \cdot g=\mathcal{D}$ is dense in $L_{p}\left(\mathbb{R}^{n}, d x\right)$, we conclude from the theorem that $\mathcal{P} \cdot g$ is dense in $L_{p}\left(\mathbb{R}^{n}, d x\right)$, as is $\operatorname{Span}\left\{e_{i \lambda} \cdot g \mid \lambda \in E\right\}$ if $E \subset \mathbb{R}^{n}$ is somewhere dense. We will later consider the case of non-smooth $g$ (see Section 6.4).

It will become apparent in Theorem 5.3 that the continuity of the maps of type $f \rightarrow f \cdot g$ from $\mathcal{O}_{w}$ into $V$ in these examples does not have to be verified. It is sufficient to observe that these maps can be defined.

For many spaces $V$ the natural action of a space $\mathcal{O}_{w}$ on $g \in V$ can be described as the transpose of an action of $\mathcal{O}_{w}$ on functions on some set $X$, the latter being defined by pointwise multiplication. In such situations a uniform formulation becomes possible, covering in one concept a variety of "appropriate" ways of multiplying objects by smooth functions. At the same time a new phenomenon appears, namely the redundancy of the hypothesis of continuity. This is due to the completeness of various spaces and it illustrates the importance of Theorem 3.22 .

Theorem 5.3. Let $X \subset \mathbb{R}^{n}$ be non-empty and let $\mathcal{T}$ be an LF-space of functions on $X$ with a defining sequence $\mathcal{T}^{1} \subset \mathcal{T}^{2} \subset \ldots$ Assume that the $\mathcal{T}^{l}$ are all $C^{\infty}\left(\mathbb{R}^{n}\right)$ modules under pointwise multiplication and suppose that convergence of a sequence in $\mathcal{T}$ implies pointwise convergence on $X$. Let $\mathcal{T}^{\prime}$ be the dual of $\mathcal{T}$, supplied with the weak topology.

Then the action of each element of $C^{\infty}\left(\mathbb{R}^{n}\right)$ on $\mathcal{T}$ is automatically continuous, and we equip $\mathcal{T}^{\prime}$ with the structure of a $C^{\infty}\left(\mathbb{R}^{n}\right)$-module by transposition.

Let $V \subset \mathcal{T}^{\prime}$ be a locally convex topological vector space and let $\mathcal{G} \subset V$ be a nonempty set of generators. Suppose that for each $g \in \mathcal{G}$ a weight $w_{g}$ on $\mathbb{R}^{n}$ is given. Assume 
1. that the topology of $V$ is weaker than the induced weak topology of $\mathcal{T}^{\prime}$ on $V$ and that $\mathcal{O}_{w_{g}} \cdot g \subset V$ for all $g \in \mathcal{G}$, or

2. that the topology of $V$ is stronger than the induced weak topology of $\mathcal{T}^{\prime}$ on $V$, that $V$ is an LF-space with a defining sequence $V^{1} \subset V^{2} \subset \ldots$ and that for each $g \in \mathcal{G}$ there exists $V^{l}$ such that $g \in V^{l}$ and $\mathcal{O}_{w_{g}} \cdot g \subset V^{l}$.

For each $g \in \mathcal{G}$ choose an admissible space $L_{g}$ for $w_{g}$. Consider the subspace $L=\sum_{g \in \mathcal{G}} L_{g} \cdot g$ of $V$. Then the following hold:

1. The closure of $L$ in $V$ is independent of the choice of the $L_{g}$.

2. The annihilator of $L$ in $V^{\prime}$ is independent of the choice of the $L_{g}$ and equal to $\bigcap_{g \in \mathcal{G}}\left(L_{g}^{\prime} \cdot g\right)^{\perp}$ for any choice of admissible spaces $L_{g}^{\prime}$.

3. The sequential closure of $L$ in $V$ is independent of the choice of the $L_{g}$ when $\mathcal{G}$ is finite.

Before giving the proof of the theorem, let us motivate its formulation by indicating what types of spaces can occur as $\mathcal{T}$ and $V$ in the theorem.

The hypotheses on the space of test functions $\mathcal{T}$ are for all non-empty open $\Omega \subset \mathbb{R}^{n}$ and all $0 \leq m \leq \infty$ satisfied by $C_{c}^{m}(\Omega)$ and $C^{m}(\Omega)$, the latter case corresponding to a constant sequence defining $\mathcal{T}$. Thus a number of common dual spaces can figure as $\mathcal{T}^{\prime}$ in the theorem and for each of these one has a variety of subspaces $V \subset \mathcal{T}^{\prime}$. As an example we consider $\mathcal{T}^{\prime}=C_{c}^{\infty}(\Omega)^{\prime}$. Then $C_{c}^{\infty}(\Omega)^{\prime}$ itself in the weak topology is covered by the first possibility. Taking the defining sequence in the second possibility equal to a fixed Fréchet space, we see that Fréchet spaces as $C^{\infty}(\Omega), L_{p}$-spaces (as well as their local versions), various types of Sobolev spaces and $\mathcal{S}$ (in case $\Omega=\mathbb{R}^{n}$ ) are all covered by this second possibility. Other spaces which are not Fréchet spaces but still LF-spaces are also covered by this second possibility. This holds e.g. for certain Sobolev spaces and for $C_{c}^{\infty}(\Omega)$. We will see in Section 6.4 that several spaces associated to a locally compact subset $X$ of $\mathbb{R}^{n}$, and notably $L_{p}$-spaces with $X$ as underlying point set, are also within the range of the theorem.

In all such spaces an inclusion of sets immediately implies equality of closure of subspaces.

Proof. We first settle the continuity of the action of $C^{\infty}\left(\mathbb{R}^{n}\right)$. Since convergence of a sequence in $\mathcal{T}$ implies pointwise convergence on $X$, the same holds for each space $\mathcal{T}^{l}$. The closed graph theorem [27, Thm. 2.15] then readily shows that the action of each fixed element of $C^{\infty}\left(\mathbb{R}^{n}\right)$ on each space $\mathcal{T}^{l}$ is continuous as a map from $\mathcal{T}^{l}$ into itself. Composed with the continuous injection $\mathcal{T}^{l} \hookrightarrow \mathcal{T}$ the action is a continuous map from each space $\mathcal{T}^{l}$ into $\mathcal{T}$. This in turn implies by general principles the continuity of the action as a map from $\mathcal{T}$ into itself, as was to be shown. We conclude that there is indeed a $C^{\infty}\left(\mathbb{R}^{n}\right)$-module structure on $\mathcal{T}^{\prime}$.

As a preparation for the conclusion of the proof, we first improve the properties of the $\mathcal{O}_{w_{g}}$. Indeed, Theorem 3.22 supplies majorants for the $w_{g}$ which still satisfy the hypotheses of the present theorem (since the corresponding function spaces are contained in the spaces $\mathcal{O}_{w_{g}}$ ) and which are of the same type, so that they have the same admissible spaces as the original $w_{g}$. Replacing the $w_{g}$ by these majorants, we may therefore assume by Theorem 3.22 that the $\mathcal{O}_{w_{g}}$ are Fréchet spaces in which convergence implies pointwise convergence on $\mathbb{R}^{n}$. 
After this preparation we note that in view of Theorem 5.1 the conclusions of the present theorem will follow once we can prove that $f \mapsto f \cdot g$ is for each $g \in \mathcal{G}$ automatically continuous as a map from $\mathcal{O}_{w_{g}}$ into $V$. To this end fix $g \in \mathcal{G}$.

Since convergence in $\mathcal{O}_{w_{g}}$ now implies pointwise convergence and $\mathcal{O}_{w_{g}}$ is now Fréchet, the closed graph theorem yields that for each fixed $t \in \mathcal{T}$, say $t \in \mathcal{T}^{l}$, the map $f \mapsto f \cdot t$ is continuous from $\mathcal{O}_{w_{g}}$ into $\mathcal{T}^{l}$ and is therefore also continuous as a map from $\mathcal{O}_{w_{g}}$ into $\mathcal{T}$. By transposition we conclude that $f_{n} \cdot g \stackrel{w}{\rightarrow} f_{\infty} \cdot g$ in the weak topology of $\mathcal{T}^{\prime}$ whenever $f_{n} \rightarrow f_{\infty}$ in $\mathcal{O}_{w_{g}}$.

The required continuity in case of the first possibility for the topology of $V$ is now immediate.

Turning to the second possibility, suppose that $g \in V^{l}$ and $\mathcal{O}_{w_{g}} \cdot g \subset V^{l}$. By continuity of the injection $V^{l} \hookrightarrow V$ it is sufficient to show that the map $f \mapsto f \cdot g$ is continuous as a map from $\mathcal{O}_{w_{g}}$ into $V^{l}$. This can be done by a final application of the closed graph theorem. Suppose then that $f_{n} \rightarrow f_{\infty}$ in $\mathcal{O}_{w_{g}}$ and that $f_{n} \cdot g \rightarrow t^{\prime}$ in $V^{l}$. We have to show that $t^{\prime}=f_{\infty} \cdot g$. Now the second convergence also holds in $V$ and, since by assumption the topology of $V$ is stronger than the induced weak topology of $\mathcal{T}^{\prime}$, we see that $f_{n} \cdot g \stackrel{w}{\rightarrow} t^{\prime}$ in the weak topology of $\mathcal{T}^{\prime}$. On the other hand, we had already observed that $f_{n} \cdot g \stackrel{w}{\rightarrow} f_{\infty} \cdot g$ in the weak topology of $\mathcal{T}^{\prime}$ whenever $f_{n} \rightarrow f_{\infty}$ in $\mathcal{O}_{w_{g}}$. By the uniqueness of weak limits in $\mathcal{T}^{\prime}$, we conclude that $t^{\prime}=f_{\infty} \cdot g$, as was to be shown. The closed graph theorem therefore does indeed apply and the proof is complete.

The following theorem has an overlap with Theorem 5.3, but it covers some important spaces like $C(X), C_{c}(X)$ and $C_{0}(X)$ for a locally compact subset $X$ of $\mathbb{R}^{n}$, spaces which are not for general $X$ covered by the previous theorem. The proof is a simpler version of the proof of Theorem 5.3 and is left to the reader.

Theorem 5.4. Let $X \subset \mathbb{R}^{n}$ be non-empty and let $V$ be an LF-space of functions on $X$ with a defining sequence $V^{1} \subset V^{2} \subset \ldots$ Suppose that convergence of a sequence in $V$ implies pointwise convergence on $X$. Let $\mathcal{G} \subset V$ be a non-empty set of generators and suppose that for each $g \in \mathcal{G}$ a weight $w_{g}$ on $\mathbb{R}^{n}$ is given. Assume that for each $g \in \mathcal{G}$ there exists $V^{l}$ such that $g \in V^{l}$ and $\mathcal{O}_{w_{g}} \cdot g \subset V^{l}$. For each $g \in \mathcal{G}$ choose an admissible space $L_{g}$ for $w_{g}$. Consider the subspace $L=\sum_{g \in \mathcal{G}} L_{g} \cdot g$ of $V$.

Then the following hold:

1. The closure of $L$ in $V$ is independent of the choice of the $L_{g}$.

2. The annihilator of $L$ in $V^{\prime}$ is independent of the choice of the $L_{g}$ and equal to $\bigcap_{g \in \mathcal{G}}\left(L_{g}^{\prime} \cdot g\right)^{\perp}$ for any choice of admissible spaces $L_{g}^{\prime}$.

3. The sequential closure of $L$ in $V$ is independent of the choice of the $L_{g}$ when $\mathcal{G}$ is finite.

Corollary 5.5. Let $V$ be as in Theorem 5.3 or 5.4 and suppose that $V$ (or each space $V^{l}$ in case of an LF-space) is a $C^{\infty}\left(\mathbb{R}^{n}\right)$-module. Let $\mathcal{G} \subset V$ be a non-empty set of generators and for $g \in \mathcal{G}$ let $L_{g}$ denote an admissible space for a holomorphic weight.

Then the closure in $V$ of the subspace $L=\sum_{g \in \mathcal{G}} L_{g} \cdot g$ is independent of the choice of the $L_{g}$.

The precise nature of $V$ and the generators is apparently irrelevant. This situation occurs e.g. for $C_{c}^{m}(\Omega), C^{m}(\Omega)(0 \leq m \leq \infty)$ and their duals, and for $C_{c}(X)$ 
and $C(X)$ and their duals for locally compact $X$. For these spaces one can for arbitrary sets of generators always switch between admissible spaces for holomorphic weights without affecting the closure.

5.2. A partial filtration associated with types of weights. Given a topological vector space $V$ as in Theorem 5.3 or Theorem 5.4 we now introduce a partial filtration on $V$ which measures for $g \in V$ both the amount of choice for admissible spaces and the minimal regularity of the Fourier transforms of associated linear functionals on $\mathcal{S}$.

For the first possibility of Theorem 5.3 let $V_{\mathcal{W}}$ denote the set of all $v \in V$ such that $\mathcal{O}_{w} \cdot v \subset V$ for some $w \in \mathcal{W}$. For $0 \leq d \leq \infty$ let $V_{d}$ denote the set of all $v \in V$ such that $\mathcal{O}_{w} \cdot v \subset V$ for some $w \in \overline{\mathcal{W}}_{d}$; the sets $V_{q a}$ and $V_{h o l}$ are defined similarly. For the second possibility of Theorem 5.3, as well as for Theorem 5.4, let $V_{\mathcal{W}}$ denote the set of all $v \in V$ such that there exists $V^{l}$ with the property that $v \in V^{l}$ and $\mathcal{O}_{w} \cdot v \subset V^{l}$ for some $w \in \mathcal{W}$. The spaces $V_{d}, V_{q a}$ and $V_{h o l}$ are defined similarly.

In all cases Proposition 3.7 implies that

$$
0 \subset V_{\text {hol }} \subset V_{q a} \subset V_{\infty} \subset V_{d} \subset V_{d^{\prime}} \subset V_{0} \subset V_{\mathcal{W}} \subset V \quad\left(d \geq d^{\prime} \geq 0\right) .
$$

One verifies that in all cases $V_{h o l}, V_{d}(0 \leq d \leq \infty)$ and $V_{\mathcal{W}}$ are vector spaces since $\mathcal{O}_{w_{1}+w_{2}} \subset \mathcal{O}_{w_{1}} \cap \mathcal{O}_{w_{2}}$ for arbitrary weights $w_{1}$ and $w_{2}$ and since the sets of weights corresponding to these subspaces of $V$ are closed under addition by Proposition 3.7. Since this latter property fails for $\mathcal{W}_{q a}$ it may well be in doubt whether $V_{q a}$ is in general closed under addition, so that we prefer to refer to (5.1) only as a partial filtration.

Definition 5.6. Let $V$ be a topological vector space as in Theorems 5.3 or 5.4 . If $g \in V_{h o l}$, where $V_{h o l}$ is as defined above, then an admissible space for $g$ is any admissible space for a holomorphic weight as in Definition 4.11. We define analogously the notion of an admissible space for elements of $V_{q a}, V_{0}$ and $V_{\mathcal{W}}$.

With this terminology the essence of Theorems 5.3 and 5.4 can be rephrased as the validity of their conclusions for any non-empty $\mathcal{G} \subset V_{\mathcal{W}}$ and any choice of admissible spaces for the elements of $\mathcal{G}$. The largest freedom of choice for a fixed $g \in \mathcal{G}$ is then determined by the leftmost set in (5.1) that still contains $g$.

We turn to Fourier transforms. In the proof of the Theorems 5.3 and 5.4 we start with $g \in V$ and a weight $w_{g}$ such that $\mathcal{O}_{w_{g}} \cdot g \subset V$ (resp. $g \in V^{l}$ and $\mathcal{O}_{w_{g}} \cdot g \subset V^{l}$ in the case of LF-spaces). The proof consists of replacing $w_{g}$ by a majorant $\widetilde{w}_{g}$ which is separated from zero on compact sets and then showing that the map $f \mapsto f \cdot g$ from $\mathcal{O}_{\widetilde{w}_{g}}$ into $V$ is necessarily continuous. Now if e.g. $g \in V_{q a}$, so that $w_{g} \in \mathcal{W}_{q a}$, then we can choose $\widetilde{w}_{g} \in \mathcal{W}_{q a}$ again. Now for all $T \in V^{\prime}$ the associated map $T_{g}: \mathcal{S} \mapsto \mathbb{C}$ defined by $\left\langle T_{g}, f\right\rangle=\langle T, f \cdot g\rangle(f \in \mathcal{S})$ is not only a tempered distribution but in fact in $\mathcal{O}_{\widetilde{w}_{g}}^{\prime}$ since it factors over $\mathcal{O}_{\widetilde{w}_{g}}$. Since $\widetilde{w}_{g} \in \mathcal{W}_{q a}$ we therefore then have knowledge from Theorem 4.9 on the minimal degree of regularity of the Fourier transform of $T_{g}$. The same argument is valid for the other classes of weights and the partial filtration (5.1) thus corresponds to various minimal degrees of regularity of the Fourier transforms $\mathcal{F}\left(T_{g}\right)$ of all these associated tempered distributions. This minimal degree of regularity of all $\mathcal{F}\left(T_{g}\right)$ for $T \in V^{\prime}$ is determined by again the leftmost set in (5.1) that still contains $g$.

To be more precise, fix $g \in V_{\mathcal{W}}$. If $g \in V_{d}(0 \leq d \leq \infty)$ then the Fourier transforms $\mathcal{F}\left(T_{g}\right)\left(T \in V^{\prime}\right)$ of the associated tempered distributions $\left\langle T_{g}, f\right\rangle=\langle T, f$. 
$g\rangle(f \in \mathcal{S})$ are all functions of class $C^{[d]}$ and given by $\widehat{T}_{g}(\lambda)=(2 \pi)^{-n / 2}\left\langle T, e_{-i \lambda} \cdot g\right\rangle$ $\left(\lambda \in \mathbb{R}^{n}\right)$. If $g \in V_{q a}$ the Fourier transforms are each in a quasi-analytic class and if $g \in V_{h o l}$ they all extend to holomorphic functions on a common tubular neighbourhood of $\mathbb{R}^{n}$. The expressions for the possible derivatives of the $\mathcal{F}\left(T_{g}\right)$ as well as estimates for these derivatives follow from those in Theorem 4.9.

\section{Applications}

In Section 5 we have given some first applications of the spaces $\mathcal{O}_{w}$ in the case of one generator. We will now consider the case of arbitrary sets of generators in a number of familiar spaces. The outline and the type of results will be quite similar for the various spaces, in accordance with our aim to develop a uniform approach.

With one exception we will use Theorem 5.3, Theorem 5.4 and Corollary 5.5 as the basic results, so that we need not verify the continuity of maps of type $f \rightarrow f \cdot g$ from a space $\mathcal{O}_{w}$ into a space of interest. A direct verification of this continuity is of course also possible. The exception is formed by the general spaces of Bernstein type in Section 6.3, a class of spaces for which one can find examples that are not covered by the aforementioned results.

With the exception of Section 6.3 we will use notations like e.g. $V_{q a}$ as in the partial filtration in Section 5.2. We will also employ the notion of admissible spaces for elements of a topological vector space as in Definition 5.6.

All subsets $\mathcal{G}$ of generators are assumed to be non-empty. If $f$ is a function, $Z(f)$ denotes its zero locus. We will also employ this notation for elements of $L_{p}$-spaces, in which case the zero locus is not necessarily well defined. Since the measure of such sets is all we will be concerned with in that situation, we will allow ourselves this imprecision. The notations concerning test functions and distributions are as in Section 2. If $y \in \mathbb{R}^{n}$ and $f$ is a function on $\mathbb{R}^{n}$ then the translate $T_{y} f$ is defined by $T_{y} f(x)=f(x-y)$.

Remark 6.1. As explained in Section 5.2 we have in applications a minimal degree of regularity of the Fourier transforms of associated tempered distributions, as well as estimates on these transforms. We will refrain from stating these results explicitly in the present section, since they are quite similar in all cases and not necessary for our purposes. It should be noted however that in other cases this extra information may be a useful additional means of investigation.

6.1. The space $\mathcal{S}$ of rapidly decreasing functions. The Schwartz space $\mathcal{S}$ is covered by the second possibility in Theorem 5.3 by taking $\mathcal{T}=\mathcal{D}$.

We start by discussing the partial filtration of Section 5.2. It is immediate that $\mathcal{S}=\mathcal{S}_{d}$ for all finite $d$ (consider $w(x)=\left(1+\|x\|^{d+1}\right)^{-1}$ ), so that $\mathcal{E}_{\text {im }}$ and all dense subspaces of $\mathcal{S}$ are admissible spaces for all $f \in \mathcal{S}$. For $\mathcal{S}_{q a}$ and $\mathcal{S}_{\text {hol }}$ the following lemma describes a class of examples.

Lemma 6.2. Let $g_{1} \in C^{\infty}\left(\mathbb{R}^{n}\right)$ and $g_{2} \in \mathcal{S}$. Assume that $\left|g_{2}\right| \in \mathcal{W}_{q a}$ (resp. $\left.\left|g_{2}\right| \in \mathcal{W}_{\text {hol }}\right)$. Suppose that for all $\alpha, \beta \in \mathbb{N}^{n}$ there exist polynomials $P_{\alpha}, Q_{\beta}$ and exponents $\mu_{\alpha}, \nu_{\beta} \geq 0$ such that

1. $\left|\partial^{\alpha} g_{1}(x)\right| \leq\left|P_{\alpha}(x)\right|\left|g_{2}\right|^{\mu_{\alpha}}$ for all $x \in \mathbb{R}^{n}$ with $\|x\|$ sufficiently large.

2. $\left|\partial^{\beta} g_{2}(x)\right| \leq\left|Q_{\beta}(x) \| g_{2}\right|^{\nu_{\beta}}$ for all $x \in \mathbb{R}^{n}$ with $\|x\|$ sufficiently large.

3. $\min _{\alpha, \beta \in \mathbb{N}^{n}}\left(\mu_{\alpha}+\nu_{\beta}\right)>0$.

Then $g=g_{1} g_{2} \in \mathcal{S}_{q a}$ (resp. $g=g_{1} g_{2} \in \mathcal{S}_{\text {hol }}$ ). If $g_{2}$ has no zeros for sufficiently large $\|x\|$ then the condition that the $\mu_{\alpha}$ and $\nu_{\beta}$ are non-negative is superfluous. 
The straightforward proof, which we leave to the reader, consists of verifying that $\mathcal{O}_{w} \cdot g_{1} g_{2} \subset \mathcal{S}$ for the quasi-analytic (resp. holomorphic) weight $w=$ $\left|g_{2}\right|^{1 / 2 \min _{\alpha, \beta \in \mathbb{N}^{n}}\left(\mu_{\alpha}+\nu_{\beta}\right)}$.

It is obvious from the lemma that $\exp \left(-\epsilon\|x\|^{2}\right) \in \mathcal{S}_{\text {hol }}$ for all $\epsilon>0$. The same is true for a more exotic example as $\sin (\exp x) \cdot \exp \left(-|x|^{2}+\sin (\exp x)\right)$ on the real line. For the quasi-analytic case the lemma provides the following relatively elementary (with $\mu_{\alpha}=0$ and $\nu_{\beta}=1$ for all $\alpha$ and $\beta$ ) examples: if $g_{2} \in \mathcal{S}$ is for large $\|x\|$ equal to one of the majorants in the first part of Proposition 3.20 and if $g_{1}$ is of polynomial growth together with its derivatives, then $g_{1} g_{2} \in \mathcal{S}_{q a}$.

With these remarks in mind we formulate the main theorem for $\mathcal{S}$. It becomes clear when one changes all admissible spaces in its formulation to $\mathcal{S}$ - as is validated by Theorem 5.3 and notes that point evaluations are continuous. The statement on supports is proved as in Lemma 6.9 below.

Theorem 6.3. Let $\mathcal{G} \subset \mathcal{S}$. For each $g \in \mathcal{G}$ choose an admissible subspace $L_{g}$ and consider the corresponding subspace $L=\sum_{g \in \mathcal{G}} L_{g} \cdot g$ of $\mathcal{S}$.

Then the annihilator of $L$ consists of all $T \in \mathcal{S}^{\prime}$ such that $g \cdot T=0$ for all $g \in \mathcal{G}$. Any such $T$ has support in $\bigcap_{g \in \mathcal{G}} Z(g)$.

The closure of $L$ is independent of the choice of the $L_{g}$ and equal to the closed multiplicative ideal generated by $\mathcal{G}$ in $\mathcal{S}$. The subspace $L$ is dense if and only if the elements of $\mathcal{G}$ have no common zero.

Corollary 6.4. Let $\mathcal{G} \subset \mathcal{S}$. Then the module generated by $\mathcal{G}$ over $\mathcal{E}_{\text {im }}$ is dense in $\mathcal{S}$ if and only if the elements of $\mathcal{G}$ have no common zero. If $\mathcal{G} \subset \mathcal{S}_{q a}$ then the analogous statement holds for the module generated over $\mathcal{P}$.

Remark 6.5. 1 . In the cyclic case we retrieve the density of $\mathcal{P} \cdot \exp \left(-\|x\|^{2}\right)$ in $\mathcal{S}$ since the gaussian has no zeros.

2. In case $L$ is not dense the natural approach in describing $\bar{L}$ is via the determination of $L^{\perp}$. If $\bigcap_{g \in \mathcal{G}} Z(g)$ is sufficiently regular the structure theorems in [29] for distributions with support in a prescribed set can then be of assistance.

The easiest case occurs when $\bigcap_{g \in \mathcal{G}} Z(g)$ has no accumulation point in $\mathbb{R}^{n}$. Then $L^{\perp}$ is as a vector space canonically isomorphic with a subspace of the direct product $\prod_{x \in \bigcap_{g \in \mathcal{G}} Z(g)} L_{x}^{\perp}$, where $L_{x}^{\perp}$ is the subspace of $L^{\perp}$ of elements supported in $\{x\}$; if $\bigcap_{g \in \mathcal{G}} Z(g)$ is finite then this isomorphism is onto. This description is computable in concrete cases and in general the isomorphism shows what the type of description of $\bar{L}$ is: a function $\psi \in \mathcal{S}$ is in $\bar{L}$ if and only if $D \psi(x)=0$ for all $x \in \bigcap_{g \in \mathcal{G}} Z(g)$ and for all constant coefficient differential operators $D$ corresponding to a (possibly infinite) base of $L_{x}^{\perp}$.

In the one-dimensional case the answer can be described explicitly, as follows. If $\bigcap_{g \in \mathcal{G}} Z(g)$ has no accumulation point in $\mathbb{R}$, then $\bar{L}$ consist precisely of those $\psi \in \mathcal{S}(\mathbb{R})$ having at all $x \in \bigcap_{g \in \mathcal{G}} Z(g)$ a zero of an order which is at least the minimum order, as $g$ ranges over $\mathcal{G}$, of the zero of $g$ at $x$.

The density statement in the following corollary parallels the well known $L_{1^{-}}$ result.

Corollary 6.6. Let $\mathcal{G} \subset \mathcal{S}$. Then the annihilator of $\operatorname{Span}\left\{T_{y} g \mid g \in \mathcal{G}, y \in \mathbb{R}^{n}\right\}$ consists precisely of the tempered distributions $T$ such that $\mathcal{F}(g) \cdot \mathcal{F}^{-1}(T)=0$ for all $g \in \mathcal{G}$. For any such $T, \mathcal{F}^{-1}(T)$ has support in $\bigcap_{g \in \mathcal{G}} Z(\mathcal{F}(g))$. The span of the 
translates of the elements of $\mathcal{G}$ is dense in $\mathcal{S}$ if and only if the Fourier transforms of the elements of $\mathcal{G}$ have no common zero in $\mathbb{R}^{n}$.

This follows immediately from Corollary 6.4 under Fourier transform. We note that if $g \in \mathcal{G}$ is such that $\mathcal{F}(g) \in \mathcal{S}_{q a}$, then one has proper subspaces of $\mathcal{E}_{\text {im }}$ which are admissible spaces for $\mathcal{F}(g)$ but correspond to spectral parameters taken from a somewhere dense set only. Correspondingly, for $g$ such that $\mathcal{F}(g) \in \mathcal{S}_{q a}$ one can restrict the translations in the above corollary to those over vectors in a somewhere dense set. By the same argument, for $\mathcal{F}(g) \in \mathcal{S}_{\text {hol }}$ the translations can be restricted still further in accordance with Definition 4.11. These various smaller sets of translations can be chosen independently for each of the elements of $\mathcal{G} \cap \mathcal{F}^{-1}\left(\mathcal{S}_{q a} \cup \mathcal{S}_{\text {hol }}\right)$ and both the description of the annihilator and the density statement in the corollary then remain valid. As an example, $\left\{T_{y} \exp \left(-x^{2}\right) \mid y \in\right.$ $E\}$ is dense in $\mathcal{S}(\mathbb{R})$ for any set $E \subset \mathbb{R}$ having an accumulation point in $\mathbb{R}$.

Corollary 6.7. Let $\mathcal{G} \subset \mathcal{S}$ be such that $\mathcal{F}(g) \in \mathcal{S}_{\text {qa }}$ for all $g \in \mathcal{G}$. Then the annihilator of the span of the derivatives of the elements of $\mathcal{G}$ consists precisely of the tempered distributions $T$ such that $\mathcal{F}(g) \cdot \mathcal{F}^{-1}(T)=0$ for all $g \in \mathcal{G}$. For any such $T, \mathcal{F}^{-1}(T)$ has support in $\bigcap_{g \in \mathcal{G}} Z(\mathcal{F}(g))$. The span of the derivatives of the elements of $\mathcal{G}$ is dense in $\mathcal{S}$ if and only if the $\mathcal{F}(g)$ have no common zero in $\mathbb{R}^{n}$.

Again the proof is immediate from Corollary 6.4.

Remark 6.8. If $g \in \mathcal{S}$ is such that $\mathcal{F}(g) \in \mathcal{S}_{q a}$, then the translates of $g$ over the vectors in a somewhere dense set span a subspace of $\mathcal{S}$ with the same closure as the span of the derivatives of $g$, as is immediate from Theorem 6.3. One can refine this if $g \in \mathcal{S}_{\text {hol }}$ and one can formulate similar results for arbitrary sets of generators. It is also clear how to obtain results on equality of the closure of the span of the derivatives of one set of generators and the closure of the span of the translates of a second set of generators. Using the continuity of the inclusion of $\mathcal{S}$ in various spaces, these results are subsequently transferred to such other spaces.

As an example on the real line, suppose that $\mathcal{G}_{1} \subset \mathcal{S}(\mathbb{R}), \mathcal{G}_{2} \subset \mathcal{S}(\mathbb{R})$ and $\mathcal{F}\left(\mathcal{G}_{2}\right) \subset$ $\mathcal{S}(\mathbb{R})_{q a}$. Assume that $\bigcap_{g_{1} \in \mathcal{G}_{1}} Z\left(\mathcal{F}\left(g_{1}\right)\right)$ and $\bigcap_{q_{2} \in \mathcal{G}_{2}} Z\left(\mathcal{F}\left(g_{2}\right)\right)$ are equal and have no accumulation point in $\mathbb{R}$, so that Remark 6.5 applies to $\left\{\mathcal{F}\left(g_{1}\right) \mid g_{1} \in \mathcal{G}_{1}\right\}$ and $\left\{\mathcal{F}\left(g_{2}\right) \mid g_{2} \in \mathcal{G}_{2}\right\}$. Then in $\mathcal{S}(\mathbb{R})$ the closure of the span of the translates of the elements of $\mathcal{G}_{1}$ is equal to the closure of the span of the derivatives of the elements of $\mathcal{G}_{2}$ if and only if for all $x \in \bigcap_{g_{1} \in \mathcal{G}_{1}} Z\left(\mathcal{F}\left(g_{1}\right)\right)$ the minimum order, as $g_{1}$ ranges over $\mathcal{G}_{1}$, of the zero of $\mathcal{F}\left(g_{1}\right)$ at $x$ is equal to the minimum order, as $g_{2}$ ranges over $\mathcal{G}_{2}$, of the zero of $\mathcal{F}\left(g_{2}\right)$ at $x$. By continuity the sufficiency of this condition persists in $L_{p}(\mathbb{R}, d x)(1 \leq p \leq \infty)$. This type of result is similar in spirit to [21], where refined results for two sets of one generator in $L_{p}$-spaces on the real line are derived.

6.2. The spaces $C_{c}^{m}(\Omega)$ and $C^{m}(\Omega)(0 \leq m \leq \infty)$ and their duals. These spaces of test functions on a non-empty open subset $\Omega$ of $\mathbb{R}^{n}$ and their duals are covered by Theorem 5.3. Since these spaces, as well as the spaces in a defining sequence for the LF-spaces among them, are all modules over $C^{\infty}\left(\mathbb{R}^{n}\right)$ we see that all admissible spaces for holomorphic weights are admissible spaces for arbitrary elements, as in Corollary 5.5.

We start with a preparatory result. 
Lemma 6.9. Suppose $0 \leq m \leq \infty$ and let $V$ denote $C_{c}^{m}(\Omega)$ or $C^{m}(\Omega)$. Then for $T \in V^{\prime}$ and $g \in V$ the following are equivalent:

1. $\langle T, \mathcal{D} \cdot g\rangle=0$.

2. $\left\langle T, C_{c}^{\infty}(\Omega) \cdot g\right\rangle=0$.

3. $\langle T, V \cdot g\rangle=0$.

If $T$ satisfies these conditions, then supp $T \subset Z(g)$.

Proof. We give the proof for $C_{c}^{m}(\Omega)$; the case $C^{m}(\Omega)$ is similar. It is trivial that (1) implies (2). Assume (2), i.e. assume that $g \cdot T \in C_{c}^{m}(\Omega)^{\prime}$ vanishes on $C_{c}^{\infty}(\Omega)$. Then by density $g \cdot T$ is identically zero on $C_{c}^{m}(\Omega)$. Thus (2) implies (3). Next assume (3) and note that $g \cdot T \in C^{m}(\Omega)^{\prime}$. By density of $C_{c}^{m}(\Omega)$ in $C^{m}(\Omega)$ we conclude from (3) that $g \cdot T=0$ in $C^{m}(\Omega)^{\prime}$. In particular, the product vanishes on the restriction of elements of $\mathcal{D}$ to $\Omega$. Hence (3) implies (1). Finally, if $\psi \in C_{c}^{\infty}(\Omega)$ and supp $\psi \cap Z(g)=\emptyset$, then $\psi$ factors as $\psi=g \cdot \chi$ for some $\chi \in C_{c}^{m}(\Omega)$. Consequently $\langle T, \psi\rangle=\langle T, g \cdot \chi\rangle=0$.

For $m=0$ the condition supp $T \subset Z(g)$ is equivalent to the other statements (see Lemma 6.22).

The equivalence of (1) and (3) in the above lemma yields the following.

Corollary 6.10. Let $V$ denote $C_{c}^{m}(\Omega)$ or $C^{m}(\Omega)$ and suppose $\mathcal{G} \subset V$ is a generating set. Then $\overline{\sum_{g \in \mathcal{G}} \mathcal{D} \cdot g}$ is equal to the closed ideal in $V$ generated by $\mathcal{G}$.

The following theorem becomes clear when one changes all admissible spaces in its formulation to $\mathcal{D}$-as is validated by Theorem 5.3 and subsequently uses Lemma 6.9, Corollary 6.10 and the continuity of point evaluations.

Theorem 6.11. Let $0 \leq m \leq \infty$. Endow $C_{c}^{m}(\Omega)$ with its usual LF-topology (resp. $C^{m}(\Omega)$ with its usual Fréchet topology). Suppose $\mathcal{G} \subset C_{c}^{m}(\Omega)$ (resp. $\mathcal{G} \subset C^{m}(\Omega)$ ). For each $g \in \mathcal{G}$ choose an admissible space $L_{g}$ for a holomorphic weight and consider the corresponding subspace $L=\sum_{g \in \mathcal{G}} L_{g} \cdot g$.

Then the annihilator of $L$ consists of all $T \in C_{c}^{m}(\Omega)^{\prime}$ (resp. all $\left.T \in C^{m}(\Omega)^{\prime}\right)$ such that $g \cdot T=0$ for all $g \in \mathcal{G}$. Any such $T$ has support in $\bigcap_{g \in \mathcal{G}} Z(g)$.

The closure of $L$ in $C_{c}^{m}(\Omega)$ (resp. $C^{m}(\Omega)$ ) is equal to the closed ideal generated by $\mathcal{G}$ in $C_{c}^{m}(\Omega)$ (resp. $C^{m}(\Omega)$ ). The subspace $L$ is dense in $C_{c}^{m}(\Omega)$ (resp. $C^{m}(\Omega)$ ) if and only if the elements of $\mathcal{G}$ have no common zero.

In the case of $C_{c}^{m}(\Omega)$ the sequential closure of $L$ is independent of the choice of the $L_{g}$ if $\mathcal{G}$ is finite.

Corollary 6.12. Suppose $\mathcal{G} \subset C_{c}^{m}(\Omega)$ (resp. $\mathcal{G} \subset C^{m}(\Omega)$ ). Then the module generated by $\mathcal{G}$ over $\mathcal{P}$ is dense in $C_{c}^{m}(\Omega)$ (resp. $C^{m}(\Omega)$ ) if and only if the elements of $\mathcal{G}$ have no common zero. The analogous statement holds for the module generated over $\mathcal{E}_{\text {im }}$.

Remark 6.13. $\quad$ 1. Specializing the corollary to $\mathcal{G}=\{1\}$ we retrieve that $\mathcal{P}$ is dense in $C^{m}(\Omega)$ because the constant function 1 has no zeros (cf. [30, p. 160]).

2. Analogously to Remark 6.5 we note that if $\bigcap_{g \in \mathcal{G}} Z(g)$ has no accumulation point in $\Omega$, then $L^{\perp}$ is as linear space canonically isomorphic to the direct sum $\bigoplus_{x \in \bigcap_{g \in \mathcal{G}} Z(g)} L_{x}^{\perp}$ in the case of $C^{m}(\Omega)$ (resp. the direct product $\prod_{x \in \bigcap_{g \in \mathcal{G}} Z(g)} L_{x}^{\perp}$ in the case of $\left.C_{c}^{m}(\Omega)\right)$, where $L_{x}^{\perp}$ is the subspace of $L^{\perp}$ of 
elements supported in $\{x\}$. Again this makes it evident that in both the cases $C_{c}^{m}(\Omega)$ and $C^{m}(\Omega)$ an element $\psi$ of the respective space is in $\bar{L}$ if and only if $D \psi(x)=0$ for all $x \in \bigcap_{g \in \mathcal{G}} Z(g)$ and for all constant coefficient differential operators $D$ corresponding to a (possibly infinite) base of $L_{x}^{\perp}$. In the one-dimensional situation one finds again that, if $\bigcap_{g \in \mathcal{G}} Z(g)$ has no accumulation point in $\Omega \subset \mathbb{R}$, then $\bar{L}$ consist precisely of those $\psi \in C_{c}^{m}(\Omega)$ (resp. $\psi \in C^{m}(\Omega)$ ) having in all $x \in \bigcap_{g \in \mathcal{G}} Z(g)$ a zero of an order which is at least the minimum order, as $g$ ranges over $\mathcal{G}$, of the zero of $g$ at $x$.

3. For finite $m$, the fact that the ideal $C^{m}(\Omega) \cdot \mathcal{G}$ is dense in $C^{m}(\Omega)$ if the generators have no common zero can also be seen to be a consequence of the results on closed ideals in $C^{m}(\Omega)$ in [32].

In [25] a necessary and sufficient condition for the density of subalgebras of $C^{m}(M)(m \geq 1)$ is given, where $C^{m}(M)$ is the algebra of functions of class $C^{m}$ on a manifold $M$ of class $C^{m}$, supplied with the topology of uniform convergence of order $m$ on compact subsets. It also follows from this condition that the ideal $C^{m}(\Omega) \cdot \mathcal{G}$ is dense in $C^{m}(\Omega)$ for finite $m \geq 1$.

4. For $m=0$ more precise results are available in Theorem 6.24.

The dual theorem is as follows.

Theorem 6.14. Let $0 \leq m \leq \infty$. Endow $C_{c}^{m}(\Omega)^{\prime}$ (resp. $\left.C^{m}(\Omega)^{\prime}\right)$ with the weak topology. Suppose $\mathcal{G} \subset C_{c}^{m}(\Omega)^{\prime}$ (resp. $\left.\mathcal{G} \subset C^{m}(\Omega)^{\prime}\right)$. For each $T \in \mathcal{G}$ choose an admissible space $L_{T}$ for a holomorphic weight and consider the corresponding subspace $\sum_{T \in \mathcal{G}} L_{T} \cdot T$.

Then the annihilator of $L$ consists of all $\psi \in C_{c}^{m}(\Omega)$ (resp. all $\psi \in C^{m}(\Omega)$ ) such that $\psi \cdot T=0$ for all $T \in \mathcal{G}$. Any such $\psi$ vanishes on $\overline{\bigcup_{T \in \mathcal{G}} \operatorname{supp} T}$, where closure is taken in $\Omega$.

The closure of $L$ in $C_{c}^{m}(\Omega)^{\prime}$ (resp. $\left.C^{m}(\Omega)^{\prime}\right)$ is independent of the choice of the $L_{T}$. Each element of $\bar{L}$ has support in $\overline{\bigcup_{T \in \mathcal{G}} \operatorname{supp} T}$. The subspace $L$ is dense in $C_{c}^{m}(\Omega)^{\prime}$ (resp. $C^{m}(\Omega)^{\prime}$ ) if and only if $\overline{\bigcup_{T \in \mathcal{G}} \operatorname{supp} T}=\Omega$.

The sequential closure of $L$ is in both cases independent of the choice of the $L_{T}$ if $\mathcal{G}$ is finite.

In the case of $C_{c}^{m}(\Omega)^{\prime}$, if the set $\mathcal{G}$ of arbitrary cardinality consists of continuous functions on $\Omega$, then the sequential closure of $L$ is independent of the choice of the $L_{T} . L$ is in this case sequentially dense if the elements of $\mathcal{G}$ have no common zero.

Proof. When changing all admissible spaces to $\mathcal{D}$ — as is validated by Theorem 5.3 all statements become clear, except the additional results on sequential closure in $C_{c}^{m}(\Omega)^{\prime}$ in the last paragraph. As to these, note that by Theorem 6.11 the closure of $L$, when considered as subspace of $C(\Omega)$ in its Fréchet topology, is independent of the choice of the $L_{T}$. The continuity of the canonical injection $C(\Omega) \hookrightarrow C_{c}^{m}(\Omega)^{\prime}$ then implies by Theorem A.2 that the sequential closure in $C_{c}^{m}(\Omega)^{\prime}$ is also independent of the choice of the $L_{T}$. If in addition the functions in $\mathcal{G}$ have no common zero, then by Theorem 6.11 $L$ is dense in $C(\Omega)$. Stated otherwise: $L$ and $C_{c}^{\infty}(\Omega)$ have equal closure in $C(\Omega)$. Again by Theorem A.2, when $L$ is considered as a subspace of $C_{c}^{m}(\Omega)^{\prime}$, it still has the same sequential closure as $C_{c}^{\infty}(\Omega)$. Since the latter space is well known to be sequentially dense in $C_{c}^{m}(\Omega)^{\prime}$, so is $L$. 
Corollary 6.15. 1. Suppose $\mathcal{G} \subset C_{c}^{m}(\Omega)^{\prime}$ (resp. $\left.\mathcal{G} \subset C^{m}(\Omega)^{\prime}\right)$. Then the modules generated by $\mathcal{G}$ over $\mathcal{P}$ and $\mathcal{E}_{\text {im }}$ are both weakly dense in $C_{c}^{m}(\Omega)^{\prime}$ (resp. $\left.C^{m}(\Omega)^{\prime}\right)$ if and only if $\overline{\bigcup_{T \in \mathcal{G}} \operatorname{supp} T}=\Omega$, where closure is taken in $\Omega$.

2. Suppose $\mathcal{G} \subset C(\Omega)$ and assume that the elements of $\mathcal{G}$ have no common zero. Then the modules generated by $\mathcal{G}$ over $\mathcal{P}$ and $\mathcal{E}_{\text {im }}$ are both sequentially dense in $C_{c}^{m}(\Omega)^{\prime}$.

Remark 6.16. $\quad 1$. Specializing to $\mathcal{G}=\{1\}$ we retrieve that $\mathcal{P}$ is sequentially dense in $C_{c}^{m}(\Omega)^{\prime}$ because the constant continuous function 1 has no zeros (cf. [30, p. 304])

2. For $m=0$ more precise results are available in Theorem 6.27 .

6.3. General spaces of Bernstein type. We will now consider a generalization of the classical Bernstein problem, both in terms of differentiability and dimensionality. The relevant spaces, of which our spaces $\mathcal{O}_{w}$ are particular examples, were introduced and studied in [33, 24]. We will generalize the results in [loc.cit.] and explain the connection with the well known sufficient condition for the polynomials to be dense in the classical continuous one-dimensional case.

First we introduce the analogue of our spaces $\mathcal{O}_{w}$ in arbitrary degree of differentiability, as follows. Suppose $m \in\{0,1,2, \ldots \infty\}$ and $w \in \mathcal{W}$. Let $C_{w}^{m}$ consist of all $f \in C^{m}\left(\mathbb{R}^{n}\right)$ such that $\lim _{\|x\| \rightarrow \infty} \partial^{\alpha} f(x) \cdot w(x)=0$ for all $\alpha \in \mathbb{N}^{n},|\alpha| \leq m$. Then $C_{w}^{m}$ becomes a locally convex space when supplied with the seminorms $p_{\alpha}(|\alpha| \leq m)$ defined by $p_{\alpha}(f)=\left\|\partial^{\alpha} f \cdot w\right\|_{\infty}\left(f \in C_{w}^{m}\right)$. The spaces $\mathcal{O}_{w}$ correspond to $m=\infty$.

Lemma 6.17. Let $0 \leq m \leq \infty$ and $w \in \mathcal{W}$. If $\mathcal{G} \subset C_{w}^{m}$ is such that $\bigcap_{g \in \mathcal{G}} Z(g)=$ $\emptyset$, then $\sum_{g \in \mathcal{G}} \mathcal{D} \cdot g$ is dense in $C_{w}^{m}$.

Proof. The injection $j: C_{c}^{m}\left(\mathbb{R}^{n}\right) \mapsto C_{w}^{m}$ is continuous and the image is dense 33. Let $j^{t}:\left(C_{w}^{m}\right)^{\prime} \mapsto C_{c}^{m}\left(\mathbb{R}^{n}\right)^{\prime}$ be the injective transposed map. If $T \in\left(C_{w}^{m}\right)^{\prime}$ vanishes on the subspace in the lemma, then for all $g \in \mathcal{G}$ we see in particular that $g \cdot j^{t}(T) \in C_{c}^{m}\left(\mathbb{R}^{n}\right)^{\prime}$ vanishes on $C_{c}^{\infty}(\Omega)$. Hence $g \cdot j^{t}(T)=0$ and therefore supp $j^{t}(T) \subset Z(g)$ for all $g$, implying that $j^{t}(T)=0$. Hence $T=0$.

The routine verification of the following lemma is left to the reader.

Lemma 6.18. Let $0 \leq m \leq \infty$ and $w \in \mathcal{W}$ and suppose $0 \leq \nu \leq 1$. Then $C_{w^{\nu}}^{m} \subset C_{w}^{m}$. If $g \in C_{w^{\nu}}^{m}$ then the assignment $f \mapsto f \cdot g$ maps $\mathcal{O}_{w^{1-\nu}}$ continuously into $C_{w}^{m}$.

Theorem 6.19. Let $0 \leq m \leq \infty$ and $w \in \mathcal{W}$. Then:

1. All admissible spaces for $w$ are dense in $C_{w}^{m}$.

2. Suppose $\mathcal{G} \subset C_{w}^{m}$ is such that $\bigcap_{g \in \mathcal{G}} Z(g)=\emptyset$. For each $g \in \mathcal{G}$ choose $0 \leq \nu_{g} \leq$ 1 such that $g \in C_{w^{\nu} g}^{m}$. Let $L_{g}$ be an admissible space for the weight $w^{1-\nu_{g}}$.

Then $\sum_{g \in \mathcal{G}} L_{g} \cdot g$ is dense in $C_{w}^{m}$.

Proof. For the first part, note that the canonical injection $C_{c}^{m}\left(\mathbb{R}^{n}\right) \hookrightarrow C_{w}^{m}$ is continuous. The image is dense [33] and we conclude that $\mathcal{D}$ is dense in $C_{w}^{m}$. The canonical injection $\mathcal{O}_{w} \hookrightarrow C_{w}^{m}$ is also continuous, so we subsequently infer from Lemma 1.1 and Theorem 4.13 that all admissible spaces for $w$ are dense in $C_{w}^{m}$.

As to the second part, for each $g \in \mathcal{G}$ Lemma 6.18 provides a continuous map from $\mathcal{O}_{w^{1-\nu_{g}}}$ into $C_{w}^{m}$ defined by sending $f$ to $f \mapsto f \cdot g$. The theorem is therefore an immediate consequence of Theorem 5.1 and Lemma 6.17. 
Taking all $\nu_{g}$ in the second part equal to e.g. $1 / 2$ we have the following consequence. The growth conditions below can be relaxed for any explicitly given weight, in particular in the second part where polynomial bounds can be replaced by the reciprocals of appropriate quasi-analytic weights.

Corollary 6.20. Let $0 \leq m \leq \infty$. Suppose $\mathcal{G} \subset C_{w}^{m}$ is such that $\bigcap_{g \in \mathcal{G}} Z(g)=\emptyset$.

1. If $w \in \mathcal{W}_{0}$ and if each of the derivatives of order at most $m$ of an arbitrary element of $\mathcal{G}$ is bounded, then the module generated by $\mathcal{G}$ over $\mathcal{E}_{\text {im }}$ is dense in $C_{w}^{m}$.

2. If $w \in \mathcal{W}_{q a}$ and if each of the derivatives of order at most $m$ of an arbitrary element of $\mathcal{G}$ is of at most polynomial growth, then the module generated by $\mathcal{G}$ over $\mathcal{P}$ is dense in $C_{w}^{m}$.

In order to establish the connection with the continuous one-dimensional Bernstein problem, consider a continuous even strictly positive weight $w: \mathbb{R} \mapsto \mathbb{R}_{\geq 0}$ with the property that $s \mapsto-\log w\left(e^{s}\right)$ is convex on $(-\infty, \infty)$ and such that

$$
\int_{0}^{\infty} \frac{\log w(t)}{1+t^{2}}=-\infty .
$$

As a consequence of the second part of Theorem $3.14 w$ is a quasi-analytic weight and we conclude from Corollary 6.20 for $\mathcal{G}=\{1\}$ that the polynomials are dense in $C_{w}^{m}$ for all $m$; moreover Theorem 6.19 asserts that the same holds for the span of the exponentials with spectral parameters in a somewhere dense subset of $\mathbb{R}^{n}$. Specializing further by letting $m=0$ one retrieves a well known sufficient condition for the polynomials to be dense in the continuous one-dimensional Bernstein space 22. 6, 16. It becomes apparent that this classical result is an aspect of a more general picture which is described by Theorem 6.19.

Let us now explain the connection with the results in [33, 24]. In [loc.cit.] the most general version of the spaces $C_{w}^{m}$ was introduced, as follows. If $m \in$ $\{0,1,2, \ldots, \infty\}$, let $\mathbf{w}=\left\{w_{\alpha}\left|\alpha \in \mathbb{N}^{n},\right| \alpha \mid \leq m\right\}$ be a set of weights on $\mathbb{R}^{n}$. These weights are assumed to be upper semi-continuous in [loc.cit.], but this can be relaxed: we will continue to assume only that these weights are bounded. Consider the space $C_{\mathbf{w}}^{m}$ of all $f \in C^{m}\left(\mathbb{R}^{n}\right)$ such that $\lim _{\|x\| \rightarrow \infty} \partial^{\alpha} f(x) \cdot w_{\alpha}(x)=0$ for all $\alpha \in \mathbb{N}^{n},|\alpha| \leq m$. The obvious choice of seminorms makes $C_{\mathbf{w}}^{m}$ into a locally convex space.

In [loc.cit.] the emphasis is on the situation where $\mathbf{w}$ is a decreasing set of weights, i.e. where for all $\alpha, \beta \in \mathbb{N}^{n}(|\alpha|,|\beta| \leq m)$ with $\alpha_{j} \geq \beta_{j}(1 \leq j \leq n)$ there exists a constant $C_{\alpha, \beta}$ such that $w_{\alpha}(x) \leq C_{\alpha, \beta} w_{\beta}(x)$ for all $x \in \mathbb{R}^{n}$. It is easily verified that in that case there is a continuous injection $C_{w_{0}}^{m} \hookrightarrow C_{\mathbf{w}}^{m}$, where $w_{0} \in \mathbf{w}$ is the weight corresponding to $\alpha=0$. Now $C_{c}^{m}\left(\mathbb{R}^{n}\right)$ is dense in both $C_{w_{0}}^{m}$ and $C_{\mathbf{w}}^{m}$ (see [33), so Lemma 1.1 implies that any dense subspace of $C_{w_{0}}^{m}$ is also dense in $C_{\mathbf{w}}^{m}$. In this way the density results in the above theorem for $C_{w_{0}}^{m}$ are transferred to $C_{\mathbf{w}}^{m}$, provided that $\mathbf{w}$ is a decreasing set of weights. One thus obtains various (not necessarily cyclic) density statements in $C_{\mathbf{w}}^{m}$. In the cyclic case one concludes e.g. that $\mathcal{P} \cdot p$ is dense in $C_{\mathbf{w}}^{m}$ for $w \in \mathcal{W}_{q a}$ and any $p \in \mathcal{P}$ without zeros on $\mathbb{R}^{n}$. Specializing still further by letting $p=1$ yields the main result in [loc.cit.], namely that $\mathcal{P}$ is dense in $C_{\mathbf{w}}^{m}$ if $\mathbf{w}$ is a decreasing set of weights such that $w_{0} \in \mathcal{W}_{q a}$.

6.4. Spaces associated with a locally compact set $X$. Throughout this section $X$ denotes a locally compact subset of $\mathbb{R}^{n}$. As a consequence of 2 , I.3.3 and 
I.9.7] such $X$ can alternatively be characterized as the intersection of an open subset $\Omega$ and a closed subset $C$ of $\mathbb{R}^{n}$. This structure equation is convenient in the proof of several statements below. We choose and fix such $\Omega$ and $C$ and assume $X=\Omega \cap C \neq \emptyset$. In our terminology a Borel measure on $X$ takes finite values on compact subsets of $X$.

Let $C_{0}(X)$ denote the continuous functions on $X$ vanishing at infinity (with respect to $X)$, supplied with the maximum norm. $C(X)$ denotes the continuous functions on $X$ with its subspace $C_{c}(X)$ of functions with compact support. These two spaces carry a standard topology which we now describe.

Choose a sequence $\left\{K_{m}\right\}_{m=1}^{\infty}$ of compact subsets of $X$ such that $X=\bigcup_{m=1}^{\infty} K_{m}$ and such that $K_{m}$ is contained in the interior (in the topology of $X$ ) of $K_{m+1}$. Define for $m=1,2, \ldots$ the seminorms $p_{m}$ on $C(X)$ by $p_{m}(f)=\max _{x \in K_{m}}|f(x)|$. Then the $p_{m}$ determine on $C(X)$ the Fréchet topology of uniform convergence on compact subsets of $X$. For $m=1,2 \ldots$ let $C_{K_{m}}(X)$ be the subspace of $C(X)$ consisting of the continuous functions on $X$ with support in $K_{m}$, supplied with the induced topology of $C(X)$. Then the $C_{K_{m}}$ form an increasing sequence of Fréchet spaces, each inducing for $m \geq 2$ on its predecessor its given topology. This sequence therefore provides $C_{c}(X)$ with an LF-topology, which is independent of the choice of the $K_{m}$. The canonical injection $C_{c}(X) \hookrightarrow C(X)$ is continuous with dense image.

The dual space $C_{c}(X)^{\prime}$ consists of the Radon measures on $X$. Such a Radon measure is locally expressible as a complex valued measure. The dual $C(X)^{\prime} \subset$ $C_{c}(X)^{\prime}$ of $C(X)$ is identified with complex Borel measures on $X$ with compact support. We supply $C_{c}(X)^{\prime}$ and $C(X)^{\prime}$ each with its weak topology.

We will be concerned with the spaces $C(X), C_{c}(X), C_{0}(X), C(X)^{\prime}$ and $C_{c}(X)^{\prime}$, as well as with $L_{p}(X, \mu)(1 \leq p<\infty)$ for an arbitrary Borel measure $\mu$ on $X$ (and its completion). For these $L_{p}$-spaces it is important to note that any relatively open subset of $X$ can be written as a countable union of compact subsets, since this holds for $\Omega$. This excludes certain measure-theoretic pathologies since it not only implies that $\mu$ is $\sigma$-finite but also that $\mu$ is automatically regular [28, Theorem 2.18].

Although the continuity hypothesis in Theorem 5.1 is easily verified in a direct fashion in the situations below, it is instructive to note that our spaces of interest are in fact covered by Theorem 5.3, Theorem 5.4 and Corollary 5.5. Indeed, Corollary 5.5 applies to the $C^{\infty}\left(\mathbb{R}^{n}\right)$-modules $C(X), C_{c}(X), C(X)^{\prime}$ and $C_{c}(X)^{\prime}$ and Theorem 5.4 handles $C_{0}(X)$. For the remaining case of $L_{p^{-}}$-spaces we note that for any Borel measure $\mu$ on $X$ the space $L_{1}^{l o c}(X, \mu)$ of locally integrable functions is canonically identified with a subspace of $C_{c}(X)^{\prime}$ via the pairing

$$
\langle f, \psi\rangle=\int_{X} f(x) \psi(x) d \mu \quad\left(f \in L_{1}^{\text {loc }}(X, \mu), \psi \in C_{c}(X)\right) .
$$

This applies in particular to $L_{p}(X, d \mu) \subset L_{1}^{\text {loc }}(X, \mu)$ for $1 \leq p \leq \infty$, so that $L_{p}(X, \mu) \hookrightarrow C_{c}(X)^{\prime}$. One verifies easily that the induced weak topology of $C_{c}(X)^{\prime}$ is weaker than the original topology of $L_{p}(X, \mu)$, so that the second possibility in Theorem 5.3 applies to $L_{p}(X, \mu)$ for $1 \leq p \leq \infty$.

This being said, we remark that our method obviously requires supplementary information on the interplay between $\mathcal{D}$ and functions on $X$. This is provided by the following three results, which are based on the structure equation $X=\Omega \cap C$.

Lemma 6.21. Taking restrictions from $\Omega$ to $X$ maps $C_{c}(\Omega)$ continuously into and onto $C_{c}(X)$. 
Proof. Let $\widetilde{\psi} \in C_{c}(\Omega)$. If $x \in X$ and $\widetilde{\psi}(x) \neq 0$ then $x \in X \cap \operatorname{supp} \widetilde{\psi}$, which is closed in $X$. Thus supp $\left.\widetilde{\psi}\right|_{X} \subset X \cap \operatorname{supp} \psi=C \cap \operatorname{supp} \widetilde{\psi}$. Since the latter set is compact the restriction map is into. It is evidently continuous. To show surjectivity, let $\psi \in C_{c}(X)$ and note that $X=\Omega \cap C$ is closed in the metrizable (hence normal) space $\Omega$. By the Tietze extension theorem [3, IX.4.2] there exists $\widetilde{\psi} \in C(\Omega)$ extending $\psi$. By a version of Urysohn's lemma [28, 2.12]) there exists $\widetilde{f} \in C_{c}(\Omega)$ which is identically 1 on supp $\psi$. Then $\widetilde{f} \widetilde{\psi} \in C_{c}(\Omega)$ extends $\psi$.

Lemma 6.22. Let $V$ denote $C_{c}(X), C(X)$ or $C_{0}(X)$. Then for $T \in V^{\prime}$ and $g \in V$ the following are equivalent:

1. $\langle T, \mathcal{D} \cdot g\rangle=0$.

2. $\left\langle T, C_{c}(X) \cdot g\right\rangle=0$.

3. $\langle T, V \cdot g\rangle=0$.

4. supp $T \subset Z(g)$.

Proof. We give the proof for $V=C(X)$; the other cases are similar. Assume (1) and consider the continuous linear functional $\psi \mapsto\langle T, \psi \cdot g\rangle$ on $C(\Omega)$. The assumption implies that in particular $\left\langle T, C_{c}^{\infty}(\Omega) \cdot g\right\rangle=0$; therefore $\langle T, C(\Omega) \cdot g\rangle=0$ by the density of $C_{c}^{\infty}(\Omega)$ in $C(\Omega)$. In particular $\left\langle T, C_{c}(\Omega) \cdot g\right\rangle=0$, which by the previous lemma is equivalent to $\left\langle T, C_{c}(X) \cdot g\right\rangle=0$. Hence (1) implies (2). Assuming (2), consider the continuous linear functional $\psi \mapsto\langle T, \psi \cdot g\rangle$ on $C(X)$. By density of $C_{c}(X)$ in $C(X)$ we conclude that (2) implies (3). It is trivial that (3) implies (1). Analogously to the proof of Lemma 6.9 one sees that (2) implies (4). There exists a complex Borel measure $\mu$ on $\mathrm{X}$ with compact support such that

$$
\langle T, f\rangle=\int_{X} f(x) d \mu(x) \quad(f \in V),
$$

which makes it clear that (4) implies e.g. (2).

Corollary 6.23. Let $V$ denote $C_{c}(X), C(X)$ or $C_{0}(X)$ and suppose $\mathcal{G} \subset V$ is a generating set. Then $\overline{\sum_{g \in \mathcal{G}} \mathcal{D} \cdot g}$ is equal to the closed ideal generated in $V$ by $\mathcal{G}$.

The following result now becomes clear from the combination of Corollary 5.5 , Lemma 6.22, (6.1) (and its local analogue for $\left.C_{c}(X)^{\prime}\right)$ and the previous corollary.

Theorem 6.24. Endow $C(X)$ with its Fréchet topology (resp. $C_{c}(X)$ with its LFtopology) and suppose $\mathcal{G} \subset C(X)$ (resp. $\mathcal{G} \subset C_{c}(X)$ ). For each $g \in \mathcal{G}$ choose an admissible space $L_{g}$ for a holomorphic weight and consider the corresponding subspace $L=\sum_{g \in \mathcal{G}} L_{g} \cdot g$.

Then the annihilator of $L$ consists of all complex Borel measures $\mu$ with compact support (resp. all Radon measures T) such that the support of $\mu$ (resp. T) is contained in $\bigcap_{g \in \mathcal{G}} Z(g)$.

The closure of $L$ in $C(X)$ (resp. $C_{c}(X)$ ) is equal to the closed ideal generated by $\mathcal{G}$ in $C(X)$ (resp. $C_{c}(X)$ ) and consists of all functions in $C(X)$ (resp. $C_{c}(X)$ ) vanishing on $\bigcap_{g \in \mathcal{G}} Z(g)$. In particular $L$ is dense in $C(X)$ (resp. $C_{c}(X)$ ) if and only if the elements of $\mathcal{G}$ have no common zero.

In the case of $C_{c}(X)$ the sequential closure of $L$ is independent of the choice of the $L_{g}$ if $\mathcal{G}$ is finite. 
Corollary 6.25. Suppose $\mathcal{G} \subset C(X)$ (resp. $\mathcal{G} \subset C_{c}(X)$ ). Then the module generated by $\mathcal{G}$ over $\mathcal{P}$ in $C(X)$ (resp. $C_{c}(X)$ ) is dense in $C(X)$ (resp. $C_{c}(X)$ ) if and only if the elements of $\mathcal{G}$ have no common zero. The analogous statement holds for the module generated over $\mathcal{E}_{i m}$.

Remark 6.26. $\quad 1$. In particular $\mathcal{P}$ is dense in $C(X)$ since the constant function 1 has no zeros. For compact $X$ we thus retrieve Weierstrass' result.

2. For compact $X$ the explicit description of the closed ideal $\bar{L}$ of the Banach algebra $C(X)$ also follows from [18, Corollary 8.3.1].

The dual theorem follows from Corollary 5.5, Lemma 6.22 and 6.1) (and its local analogue for $\left.C_{c}(X)^{\prime}\right)$. The last statement in the theorem is demonstrated by considering $C(X)$, analogously to the proof of Theorem 6.14.

Theorem 6.27. Endow $C(X)^{\prime}$ (resp. $\left.C_{c}(X)^{\prime}\right)$ with the weak topology. Suppose $\mathcal{G} \subset C(X)^{\prime}$ (resp. $\left.\mathcal{G} \subset C_{c}(X)^{\prime}\right)$. For each $T \in \mathcal{G}$ choose an admissible space $L_{T}$ for a holomorphic weight and consider the corresponding subspace $L=\sum_{T \in \mathcal{G}} L_{T} \cdot T$ of compactly supported complex Borel measures (resp. Radon measures).

Then the annihilator of $L$ consists of all $\psi \in C(X)$ (resp. all $\psi \in C_{c}(X)$ ) such that $\psi$ vanishes on $\overline{\bigcup_{T \in \mathcal{G}} \operatorname{supp} T}$, where closure is taken in $X$.

The closure of $L$ in $C(X)^{\prime}$ (resp. $\left.C_{c}(X)^{\prime}\right)$ consists of all compactly supported complex Borel measures (resp. consists of all Radon measures) $S$ in $C(X)^{\prime}$ (resp. $\left.C_{c}(X)^{\prime}\right)$ such that $\operatorname{supp} S \subset \overline{\bigcup_{T \in \mathcal{G}} \operatorname{supp} T}$. The subspace $L$ is dense in $C(X)^{\prime}$ (resp. $\left.C_{c}(X)^{\prime}\right)$ if and only if $\overline{\bigcup_{T \in \mathcal{G}} \operatorname{supp} T}=X$.

The sequential closure of $L$ is in both cases independent of the choice of the $L_{T}$ if $\mathcal{G}$ is finite. In the case of $C_{c}(X)^{\prime}$, if the set $\mathcal{G}$ of arbitrary cardinality consists of continuous functions on $X$, then the sequential closure of $L$ is independent of the choice of the $L_{T}$.

Corollary 6.28. Suppose $\mathcal{G} \subset C(X)^{\prime}$ (resp. $\left.\mathcal{G} \subset C_{c}(X)^{\prime}\right)$. Then the module generated by $\mathcal{G}$ over $\mathcal{P}$ is weakly dense in $C(X)^{\prime}$ (resp. $\left.C_{c}(X)^{\prime}\right)$ if and only if $\overline{\bigcup_{T \in \mathcal{G}} \operatorname{supp} T}=X$, where closure is taken in $X$. The analogous statement holds for the module generated over $\mathcal{E}_{\text {im }}$.

We now consider $C_{0}(X)$ and discuss the partial filtration of Section 5.2. Evidently $C_{0}(X) \subset C_{0}(X)_{\mathcal{W}}$ by considering the weight $w=1$. In fact $C_{0}(X)=$ $C_{0}(X)_{0}$. To see this, let $g \in C_{0}(X)$. For $x \in \mathbb{R}^{n}$ let $w(x)=|g|^{1 / 2}$ if $x \in X$ and $w(x)=0$ if $x \notin X$. Then $w \in \mathcal{W}_{0}$ and $\mathcal{O}_{w} \cdot g \subset C_{0}(X)$. Furthermore if $g \in C_{0}(X)$ and if there exists a weight $w$ in $\mathcal{W}_{d}$ for some $0<d \leq \infty$ (resp. a weight $w$ in $\mathcal{W}_{q a}$, resp. a weight $w$ in $\mathcal{W}_{\text {hol }}$ ) which is strictly positive on $X$ and such that $w^{-1} \cdot g$ vanishes at infinity (with respect to $X$ ), then $g \in C_{0}(X)_{d}$ (resp. $g \in C_{0}(X)_{q a}$, resp. $\left.g \in C_{0}(X)_{h o l}\right)$ since $\mathcal{O}_{w} \cdot f \subset C_{0}(X)$.

For $X=\mathbb{R}^{n}$ we note the following explicit example: if $g$ is continuous on $\mathbb{R}^{n}$ and if $|g|$ is equal to one of the majorants in the first part of Proposition 3.20 for sufficiently large $\|x\|$, then $g \in C_{0}\left(\mathbb{R}^{n}\right)_{q a}$. This follows from considering the quasianalytic weight $w$ given by $w=|g|^{1 / 2}$ for large $\|x\|$ and defined to be equal to 1 at the remaining part of $\mathbb{R}^{n}$.

The following theorem follows from Theorem 5.4, Lemma 6.22, Corollary 6.23 and the obvious analogue of (6.1). Here, as with $C(X)$, it is also possible to resort to Banach algebra theory for the explicit description of the closure once this closure has been identified as an ideal. 
Theorem 6.29. Let $\mathcal{G} \subset C_{0}(X)$. For each $g \in \mathcal{G}$ choose an admissible subspace $L_{g}$ and consider the corresponding subspace $L=\sum_{g \in \mathcal{G}} L_{g} \cdot g$.

Then the annihilator of $L$ consists of all complex Borel measures $\mu$ such that the support of $\mu$ (resp. $T$ ) is contained in $\bigcap_{g \in \mathcal{G}} Z(g)$.

The closure of $L$ is equal to the closed ideal generated by $\mathcal{G}$ in $C_{0}(X)$ and consists of all functions in $C_{0}(X)$ vanishing on $\bigcap_{g \in \mathcal{G}} Z(g)$. The subspace $L$ is dense in $C_{0}(X)$ if and only if the elements of $\mathcal{G}$ have no common zero.

Corollary 6.30. Let $\mathcal{G} \subset C_{0}(X)$. Then the module generated by $\mathcal{G}$ over $\mathcal{E}_{\text {im }}$ is dense in $C_{0}(X)$ if and only if the elements of $\mathcal{G}$ have no common zero. If $\mathcal{G} \subset$ $C_{0}(X)_{q a}$ then the analogous statement holds for the module generated over $\mathcal{P}$.

We turn to the spaces $L_{p}(X, \mu)(1 \leq p \leq \infty)$ for a Borel measure $\mu$ on $X$. As with $C_{0}(X)$ we have $L_{p}(X, \mu) \subset L_{p}(X, \mu)_{\mathcal{W}}$. If $g \in L_{p}(X, \mu)$ and if there exists a weight $w$ in $\mathcal{W}_{d}$ for some $0 \leq d \leq \infty$ (resp. a weight $w$ in $\mathcal{W}_{q a}$, resp. a weight $w$ in $\mathcal{W}_{\text {hol }}$ ) such that $w^{-1} \cdot g \in L_{p}(X, \mu)$, then $g \in L_{p}(X, \mu)_{d}$ (resp. $g \in L_{p}(X, \mu)_{q a}$, resp. $\left.g \in L_{p}(X, \mu)_{h o l}\right)$.

For $X=\mathbb{R}^{n}$ we note the following explicit example: if $g$ is Borel measurable, if $|g|$ is equal to one of the majorants in the first part of Proposition 3.20 for sufficiently large $\|x\|$, and if $|g|^{\nu} \in L_{p}\left(\mathbb{R}^{n}, \mu\right)$ for some $0 \leq \nu<1$, then $g \in L_{p}\left(\mathbb{R}^{n}, \mu\right)_{q a}$. This follows from considering the quasi-analytic weight $w$ given by $w(x)=|g|^{1-\nu}$ for sufficiently large $\|x\|$ and defined to be equal to 1 at the remaining part of $\mathbb{R}^{n}$.

Theorem 6.31. Let $\mu$ be a Borel measure on $X$ and suppose $\mathcal{G} \subset L_{p}(X, \mu)(1 \leq$ $p<\infty)$ is countable. For each $g \in \mathcal{G}$ choose an admissible subspace $L_{g}$ and consider the corresponding subspace $L=\sum_{g \in \mathcal{G}} L_{g} \cdot g$.

Then the annihilator of $L$ consists of all $f \in L_{q}(X, \mu)$ vanishing $\mu$-almost everywhere on the complement of the common zero locus of the $g \in \mathcal{G}$. Here $q$ is the conjugate exponent of $p$.

The closure of $L$ consists of all $g \in L_{p}(X, \mu)$ vanishing $\mu$-almost everywhere on the common zero locus of the $g \in \mathcal{G}$. The subspace $L$ is dense in $L_{p}(X, \mu)$ if and only if the $g \in \mathcal{G}$ have $\mu$-almost no common zeros.

Proof. In view of Theorem 5.3 the annihilator of $L$ can be identified with those $f \in L_{q}(X, \mu)$ such that

$$
\int_{X} \psi(x) f(x) g(x) d \mu(x)=0
$$

for all $g \in \mathcal{G}$ and $\psi \in \mathcal{D}$. Choose such $f$ and fix $g \in \mathcal{G}$. Then the left hand side in (6.2) defines a continuous functional on $C_{c}(\Omega)$ which vanishes on $C_{c}^{\infty}(\Omega)$. By density we conclude that (6.2) holds for all $\psi \in C_{c}(\Omega)$ or, as is equivalent by Lemma 6.21, for all $\psi \in C_{c}(X)$. Consider the complex Borel measure $\nu$ on $X$ defined by

$$
\nu(E)=\int_{E} f(x) g(x) d \mu(x)
$$

for Borel sets E. From the Radon-Nikodym theorem and (6.2) we have

$$
\int_{X} \psi(x) d \nu(x)=0 \quad\left(\psi \in C_{c}(X)\right) .
$$


By the Riesz representation theorem this implies that the total variation of $\nu$ is zero, i.e.

$$
\int_{X}|f(x) g(x)| d \mu(x)=0 .
$$

Therefore $f g=0$ a.e. $(\mu)$. Since $g \in \mathcal{G}$ was arbitrary we conclude that $f$ vanishes $\mu$-almost everywhere on the complement of the common zero locus of the $g \in \mathcal{G}$. The converse statement for the annihilator is obvious. As to $\bar{L}$, the necessity of the condition on the zero locus follows from the fact that a convergent sequence in an $L_{p}$-space has a subsequence that converges almost everywhere. The sufficiency is evident from the description of $L^{\perp}$.

Remark 6.32. Let $\mu^{*}$ be the completion of $\mu$ as obtained by including the subsets of sets of measure zero in the domain of definition of the set function. By [11, p. 56, Theorem C] this completion $\mu^{*}$ can alternatively be defined as the result of the Caratheodory extension procedure. Then the theorem also holds for $\mu^{*}$ since $L_{p}(X, \mu)$ and $L_{p}\left(X, \mu^{*}\right)$ are canonically isomorphic as a consequence of 28, p. 143, Lemma 1].

Corollary 6.33. Let $\mu$ be a Borel measure on $X$ or the completion of such a measure and let $1 \leq p<\infty$. Let $\mathcal{G} \subset L_{p}(X, \mu)_{q a}$ be countable. Then the module generated by $\mathcal{G}$ over $\mathcal{P}$ is dense in $L_{p}(X, \mu)$ if and only if the $g \in \mathcal{G}$ have $\mu$-almost no common zeros. The analogous statement holds for the module generated over $\mathcal{E}_{\text {im }}$ by a countable subset $\mathcal{G}$ of $L_{p}(X, \mu)_{0}$.

Corollary 6.34. Let $\mu$ be a Borel measure on $X$ or the completion of such a measure. Suppose

$$
\int_{X} w(x)^{-1} d \mu<\infty
$$

for some quasi-analytic weight $w$ which is measurable on $X$. Then $\mathcal{P}$ is dense in $L_{p}(X, \mu)(1 \leq p<\infty)$ and the same holds for the span of the trigonometric functions $e_{i \lambda}$ corresponding to spectral parameters in a somewhere dense set.

Proof. Replacing $w$ by a majorant as in Theorem 3.22 we may assume that $w$ is strictly positive. Note that then $\mathcal{O}_{w^{1 / p}} \cdot 1 \subset L_{p}(X, \mu)$. Since $w^{1 / p} \in \mathcal{W}_{q a}$ we conclude that $1 \in L_{p}(X, \mu)_{q a}$. The constant function 1 has no zeros and the density statements therefore follow from Theorem 6.31.

For $X=\mathbb{R}^{n}, p=2$ and the choice of a holomorphic weight $\exp (-\epsilon\|x\|)$ for some $\epsilon>0$ the polynomial part of the corollary specializes to the assertion that the polynomials are dense in $L_{2}\left(\mathbb{R}^{n}, \mu\right)$ if

$$
\int_{\mathbb{R}^{n}} e^{\epsilon\|x\|} d \mu<\infty
$$

for some $\epsilon>0$. This is the multidimensional analogue [8, Theorem 3.1.18] of Hamburger's theorem [12]. According to the corollary, the result can be strengthened, not only with respect to the underlying point set, the trigonometric functions and other values than $p=2$, but most notably the integrability condition can be relaxed. The integrand in (6.3) can e.g. be replaced by any positive function which 
at infinity is equal to $\exp (\epsilon\|x\| / \log a\|x\|)$ for some $\epsilon, a>0$. The other examples of quasi-analytic weights in Section 3.4 provide even more lenient integrability conditions.

6.5. Determinate multidimensional measures. As an application of the preceding section we have the following result.

Theorem 6.35. Let $\mu$ be a probability measure on $\mathbb{R}^{n}$ such that

$$
\int_{\mathbb{R}^{n}} w(x)^{-1} d \mu<\infty
$$

for some measurable quasi-analytic weight. Then $\mu$ is determinate. Furthermore $\mathcal{P}$ is then dense in $L_{p}\left(\mathbb{R}^{n}, \mu\right)$ for all $1 \leq p<\infty$ and the same holds for the span of the trigonometric functions $e_{i \lambda}$ corresponding to spectral parameters in a somewhere dense set.

Proof. The density is a special case of Corollary 6.34. We see that there exists $p_{0}>2$ such that $\mathcal{P}$ is dense in $L_{p_{0}}\left(\mathbb{R}^{n}, \mu\right)$. It is known that $\mu$ is then determinate 9.

Note that the criterion in the above theorem is directly in terms of the measure and circumvents e.g. the computation of moments. For the choice of a holomorphic weight $\exp (-\epsilon\|x\|)$ for some $\epsilon>0$ the condition specializes to (6.3) and the corresponding result is known [8, Theorem 3.1.17], but as with the density of $\mathcal{P}$ in Corollary 6.34 we see that the classical condition $(6.3)$ can be relaxed.

The determinacy of the measure can also be proved directly as an application of an extended Carleman theorem in arbitrary dimension, using the classification of quasi-analytic weights on $\mathbb{R}^{n}$. We expect to report separately on this.

\section{INTERPRETATION IN TERMS OF LIE GROUPS}

We will now review the approach of the density results in Section 1 in a formalism that indicates how the setup can be generalized to certain classes of connected Lie groups. It will become apparent that such a generalization yields results situated on the unitary dual of a Lie group rather than on the group itself. It also shows that the results on $\mathbb{R}^{n}$ as we have derived them are actually results on $\widehat{\mathbb{R}}^{n}$.

Given the indicative character of this section we are not precise about the various topologies that one has to impose in order to validate the line of reasoning below. The case of $\mathbb{R}^{n}$ in the present paper does not provide too much information on this aspect, since various notions as well as topologies coincide in that situation. We therefore concentrate on the formal structure and leave a possible further concretization on basis of a more thorough investigation to the interested reader.

For background on the notions from Lie theory the reader is referred to e.g. 17, 31.

7.1. Formalism. Let $G$ be a connected Lie group with unit element $e$, universal enveloping algebra $\mathfrak{U}(G)$ and space of compactly supported smooth functions $\mathcal{D}(G)$. Suppose $\left\{\left(\pi_{\lambda}, H_{\lambda}\right)\right\}_{\lambda \in \Lambda}$ is a collection of unitary representations $\pi_{\lambda}$ of $G$ on Hilbert spaces $H_{\lambda}$. Let $\Lambda^{\infty}=\coprod_{\lambda \in \Lambda} H_{\lambda}^{\infty}$ denote the disjoint union of the smooth vectors in the various representations and put End $\Lambda^{\infty}=\coprod_{\lambda \in \Lambda}$ End $H_{\lambda}^{\infty}$, the disjoint union of the linear transformations of these spaces of smooth vectors. One has the naturally associated vector space $\Gamma \Lambda^{\infty}$ of sections of $\Lambda^{\infty}$ and the algebra $\Gamma$ End $\Lambda^{\infty}$ 
of sections of End $\Lambda^{\infty}$. We let $\Gamma$ End $\Lambda^{\infty}$ act on $\Gamma \Lambda^{\infty}$ by the obvious action in each fiber.

In $\Gamma$ End $\Lambda^{\infty}$ we have three natural subspaces - in fact subalgebras - as images of a Fourier transform:

1. For $g \in G$ define its Fourier transform $\widehat{g} \in \Gamma$ End $\Lambda^{\infty}$ by $\widehat{g}(\lambda)=\pi_{\lambda}(g)$. The Fourier transform extends to the group algebra $\mathbb{C}[G]$.

2. For $D \in \mathfrak{U}(G)$ define its Fourier transform $\widehat{D} \in \Gamma$ End $\Lambda^{\infty}$ by $\widehat{D}(\lambda)=\pi_{\lambda}(D)$, where we let $\pi_{\lambda}$ also denote the associated representation of $\mathfrak{U}(G)$ on $H_{\lambda}^{\infty}$.

3. For $f \in \mathcal{D}(G)$ define its Fourier transform $\widehat{f} \in \Gamma$ End $\Lambda^{\infty}$ by $\widehat{f}(\lambda)=$ $\int_{G} f(g) \pi_{\lambda}(g) d g$.

Suppose that the three subalgebras $\widehat{\mathfrak{U}}(G), \widehat{\mathbb{C}}[G]$ and $\widehat{\mathcal{D}}(G)$ of $\Gamma$ End $\Lambda^{\infty}$ are all contained in some subspace $\mathcal{O}$ of $\Gamma$ End $\Lambda^{\infty}$. We assume that $\mathcal{O}$ carries a Fréchet topology and aim at proving that these subspaces of $\mathcal{O}$ (or only the second and the third to start with) have equal closure in $\mathcal{O}$. We do this by showing that they are all dense. The crucial tool for this, as we will see, is the Fourier transform $\mathcal{F}: G \mapsto \mathcal{O}$ defined by $\mathcal{F}(g)=\widehat{g}$.

Before outlining the possible proof we illustrate how this setup corresponds to the case of $\mathbb{R}^{n}$ in Section 4 . In case of $\mathbb{R}^{n}$ we choose $\Lambda=\mathbb{R}^{n}$ (actually one should write $\Lambda=\widehat{\mathbb{R}}^{n}$ ) with $\pi_{\lambda}$ equal to the one-dimensional irreducible unitary representation with spectral parameter $\lambda$. In this situation matters are simplified — or perhaps obscured - since both $\Gamma \Lambda^{\infty}$ and $\Gamma$ End $\Lambda^{\infty}$ can be identified with functions on $\mathbb{R}^{n}$, the action of the latter on the former being pointwise multiplication. Under this identification pointwise multiplication by elements of $\mathcal{E}_{\text {im }}$ now corresponds to $\widehat{\mathbb{C}}[G]$, but with an important difference: the role of the variables has been reversed compared to Section 1 . Namely, in the present picture the variable in the underlying point set $\Lambda$ is the parameter for the unitary dual of $\mathbb{R}^{n}$, i.e. $\lambda$. Similarly, the image of the universal enveloping algebra of $\mathbb{R}^{n}$ under the present Fourier transform now corresponds to pointwise multiplication by elements of $\mathcal{P}$ (with $\lambda$ as variable). The counterpart of $\mathcal{O}$ is pointwise multiplication by the elements of one of our spaces $\mathcal{O}_{w}$ (which in our approach could as well be assumed to be Fréchet, as we have seen). The definition of $\mathcal{F}$ in the general context corresponds in case of $\mathbb{R}^{n}$ to the map $x \mapsto\{\lambda \rightsquigarrow \exp i(\lambda, x)\}$. This is the map $\psi_{1}$ of Section 1 , but again there is a reversal of roles for $\lambda$ and $x$.

The role of the space $\mathcal{D}\left(\mathbb{R}^{n}\right)$ as the basic dense subspace of $\mathcal{O}_{w}$ is now most naturally taken over by $\widehat{\mathcal{D}}(G)$. In the case of $\mathbb{R}^{n}$ the latter space does not coincide with $\mathcal{D}\left(\mathbb{R}^{n}\right)$, but $\widehat{\mathcal{D}}\left(\mathbb{R}^{n}\right)$ is dense in $\mathcal{O}_{w}$ just as well (here the range result from Fourier analysis is essential!), so we could equivalently have worked with this space from the very beginning. Conceptually it is, as will become apparent, actually the natural space to work with, but in case of $\mathbb{R}^{n}$ we preferred to use $\mathcal{D}\left(\mathbb{R}^{n}\right)$ as the basic subspace since that is the most practical space for the applications on $\mathbb{R}^{n}$.

As a parallel to $\mathbb{R}^{n}$, in the general case we make the fundamental assumption that we have already been able to show that $\widehat{\mathcal{D}}(G)$ is dense in $\mathcal{O}$. This can analogously to $\mathbb{R}^{n}$ be expected to be basically a range result from harmonic analysis for $G$.

Let us now indicate how one could prove in this general setup that $\overline{\widehat{C}[G]}=\overline{\widehat{\mathcal{D}}(G)}$ under the assumption that $\mathcal{F}: G \mapsto \mathcal{O}$ is continuous. For $\mathbb{R}^{n}$ this corresponds to proving that $\overline{\mathcal{E}_{\text {im }}}=\overline{\widehat{\mathcal{D}}}$ in $\mathcal{O}_{w}$ if $w \in \mathcal{W}_{0}$, since under the latter condition on the weight we established in Proposition 4.5 that $\psi_{1}: \mathbb{R}^{n} \mapsto \mathcal{O}_{w}$ is continuous. 
Consider for $f \in \mathcal{D}(G)$ the weak integral

$$
I_{f}=\int_{G} f(g) \widehat{g} d g,
$$

which exist in the Fréchet space $\mathcal{O}$. Suppose $T \in \mathcal{O}^{\prime}$ vanishes on $\widehat{\mathbb{C}}[G]$. Then $T$ vanishes on all weak integrals $I_{f}$. On the other hand, we have at least formally that $I_{f}(\lambda)=\widehat{f}(\lambda)$ for all $\lambda \in \Lambda$, i.e. $I_{f}=\widehat{f}$. Therefore such $T$ vanishes on $\widehat{f}$ for all $f \in \mathcal{D}(G)$, i.e. on a subspace which we had assumed to be dense in $\mathcal{O}$. Hence $T=0$ as required.

Suppose next that we want to prove that $\overline{\widehat{\mathfrak{U}}(G)}=\overline{\widehat{\mathbb{C}}[G]}=\overline{\widehat{\mathcal{D}}(G)}$. For $\mathbb{R}^{n}$ this corresponds to $\overline{\mathcal{P}}=\overline{\mathcal{E}_{\mathrm{im}}}=\overline{\widehat{\mathcal{D}}}$ in $\mathcal{O}_{w}$, which we were able to demonstrate for $w \in$ $\mathcal{W}_{q a}$. Let us assume that $\mathcal{F}: G \mapsto \mathcal{O}$ is smooth, which we concluded in case of $\mathbb{R}^{n}$ for $\psi_{1}$ in Corollary 4.7, provided $w \in \mathcal{W}_{\infty}$. Then we already have $\overline{\widehat{\mathbb{C}}[G]}=\overline{\widehat{\mathcal{D}}(G)}$ by the previous argument. In addition one verifies that formally the following relation holds:

$$
D(T \circ \mathcal{F})(g)=\langle T, \widehat{g} \circ \widehat{D}\rangle \quad\left(g \in G, D \in \mathfrak{U}(G), T \in \mathcal{O}^{\prime}\right) .
$$

The necessary additional ingredient is now a theorem asserting that $T \circ \mathcal{F}$ has the quasi-analytic property at $e \in G$ for all $T \in \mathcal{O}^{\prime}$. Indeed, if in that case $T \in \mathcal{O}^{\prime}$ vanishes on $\widehat{\mathfrak{U}}(G) \subset \mathcal{O}$ we conclude from (7.1) that $D(T \circ \mathcal{F})(e)=0$ for all $D \in \mathfrak{U}(G)$. The hypothesized quasi-analytic property then yields that $T \circ \mathcal{F}=0$, implying that $T$ vanishes on $\widehat{\mathbb{C}}[G]$. We had already concluded that the latter space is dense in $\mathcal{O}$; therefore $T=0$ as required.

For the sake of completeness, let us note that for $T \in \mathcal{O}^{\prime}$ the map $g \mapsto\langle T, \widehat{g}\rangle$ is a function on $G$ which (up to normalization and a minus sign) was identified in Section 1 as the Fourier transform of the tempered distribution $T$ on $\mathbb{R}^{n}$. In the general setup $T$ is now understood as a tempered distribution on $\widehat{\mathbb{R}}^{n}$. In the general case the identification of the corresponding function appears to have no natural analogue, reflecting our observation in the case of $\mathbb{R}^{n}$ (cf. Remark 4.14) that the identification of the function $\widehat{T}$ as a Fourier transform is not essential, but that a range result from harmonic analysis cannot be dispensed with.

The above framework constitutes the formal generalization to a connected Lie group $G$ of our approach to the spaces $\mathcal{O}_{w}$. Once this outline has been completed for a particular group, one can exploit results on equal closure obtained in such spaces $\mathcal{O} \subset \Gamma$ End $\Lambda^{\infty}$ to obtain closure results in topological vector spaces $V \subset \Gamma \Lambda^{\infty}$ by applying Theorem A.2 to the fiberwise action of the elements of $\mathcal{O}$ on the elements of $V$, provided that the necessary continuity hypotheses are satisfied by this action. Evidently $V$ can also consist of equivalence classes of sections in $\Gamma \Lambda^{\infty}$ (the analogue of e.g. $L_{p}$-spaces) or the action can be transposed to dual objects (the analogue of spaces of distributions and measures).

The formalism as indicated explains at a conceptual level the intimate connection between the spaces $\mathcal{E}_{\mathrm{im}}$ and $\mathcal{P}$ in parts of classical approximation theory: the former space is the group algebra of $\mathbb{R}^{n}$ and the latter is the universal enveloping algebra of $\mathbb{R}^{n}$, both acting on the smooth vectors in the various irreducible unitary representations of the group. It is now also apparent why these and other of the subspaces of $\mathcal{O}_{w}$ that we encountered are actually algebras. We did not use the multiplicative structure of such spaces, although it allowed a convenient terminology in terms of modules, but it is now evident that these spaces are the image 
(or the closure of an image) of naturally occurring algebras in Lie theory under a Fourier transform. Therefore these subspaces of $\mathcal{O}_{w}$ had to be algebras themselves.

The outline above makes it obvious that generalizations of our results on $\mathbb{R}^{n}$ can only be expected to be situated on sets of representation of groups. Since abelian groups can be viewed as unitary duals, such groups form an exceptional class in the sense that one can obtain results situated on these groups themselves. From the present point of view this is a coincidence. The case of the self-dual $\mathbb{R}^{n}$ is in a sense particularly deceptive since a maximal number of various different structures corresponding to the general framework then coincide.

7.2. Perspectives. We now make some tentative comments on the practical feasibility and context of the above outline.

First of all, a technical point of some importance is the quasi-analytic property on Lie groups: what conditions on a smooth function $f$ on a connected Lie group $G$ are sufficient to ensure $f$ vanishes identically if $D f(e)=0$ for all $D \in \mathfrak{U}(G)$ ? If the exponential map is surjective then one can in principle resort to $\mathbb{R}^{n}$ for knowledge on quasi-analytic classes. In the case of arbitrary $G$ one can hope to be able to use the local character of theorems in the vein of Theorem B.1. A theorem on quasi-analytic classes on general Lie groups however does not seem to be available at present.

As to the practical context of the general scheme, it stands to reason that additional conditions on $\Lambda$ and the various topologies are necessary to obtain meaningful applications. The case of $\mathbb{R}^{n}$ provides the following clues.

First of all, our definition of the spaces $\mathcal{O}_{w}$ for $\mathbb{R}^{n}$ involved derivatives (now with respect to $\lambda$ ) and the behaviour at infinity. This suggests that the representations corresponding to the elements of $\Lambda$ should be related to each other and to a topology on $\Lambda$ - as was only to be expected.

More interesting is the following trivial application of our method for $\mathbb{R}^{n}$. Let $c_{1}, c_{2} \in \mathbb{C}$ and $\lambda_{0} \in \mathbb{R}^{n}$ be such that $c_{1} \neq 0$. Choose $f_{1} \in \mathcal{S}_{q a}$ (see Section 6.1 for notation) without zeros and such that $f_{1}\left(\lambda_{0}\right)=c_{1}$. This is possible since $c_{1} \neq 0$. Choose $f_{2} \in \mathcal{S}$ such that $f_{2}\left(\lambda_{0}\right)=c_{2}$. Then $\mathcal{P} \cdot f_{1}$ is dense in $\mathcal{S}$ as a consequence of the results in Section 6.1. In particular, if $\epsilon>0$ is given then there exists a polynomial $P_{\epsilon}$ such that $\left\|f_{1}-P_{\epsilon} \cdot f_{2}\right\|_{\infty}<\epsilon$. Specializing to $\lambda_{0}$ yields $\left|c_{2}-P_{\epsilon}\left(\lambda_{0}\right) \cdot c_{1}\right|<\epsilon$. One can obviously replace the Schwartz space by other spaces of functions and obtain the same result in a similar fashion. This result, though trivial for $\mathbb{R}^{n}$, has an interpretation in the formalism for the general case. By virtue of its type of proof, which will have an obvious analogue if meaningful applications as in the case of $\mathbb{R}^{n}$ are possible, it can now be interpreted as the density in $H_{\lambda_{0}}^{\infty}$ of the orbit of any non-zero smooth vector under the universal enveloping algebra, and this for arbitrary $\lambda_{0} \in \Lambda$. Since $H_{\lambda_{0}}^{\infty}$ is in turn dense in $H_{\lambda_{0}}$ and since the above density of the orbit is in particular valid for analytic vectors, one is led to the conclusion that the analytic vectors are dense in each $H_{\lambda_{0}}$, i.e. that all $\pi_{\lambda}(\lambda \in \Lambda)$ have to be irreducible. This locates the spaces $\mathcal{O}$ on the unitary dual of $G$. Perhaps it is already sufficient to assume that he $\pi_{\lambda}$ are only generically irreducible, thus admitting a somewhat wider context.

In view of all the above we tentatively expect that a generalization of our method can be established for (a subset consisting of irreducible representations in) a series of (possibly induced) unitary representations for reductive groups. The duals of 
connected nilpotent groups appear to be another group of candidates. Once established, relatively easy applications as in Section 6 are within reach, each situated on subsets of the unitary dual or perhaps (if the representations are only generically irreducible) on slightly larger sets. We leave it to the reader to determine whether the relative ease of the possible applications merits the effort of establishing the generalization in particular cases of interest.

To conclude we note that the above formalism in principle still admits a further generalization along the same lines to e.g. non-unitary Hilbert representations or Fréchet representations and their smooth vectors.

7.3. The $n$-torus. The general outline can obviously be completed for the $n$-torus $\mathbb{T}^{n}$, yielding results situated on $\mathbb{Z}^{n}$. Since $\widehat{\mathbb{T}}^{n} \subset \widehat{\mathbb{R}}^{n}$ non-trivial applications can already be obtained from the results for $\mathbb{R}^{n}$ in the present paper, simply by letting the spaces $\mathcal{O}_{w}$ act on spaces of functions on $\mathbb{Z}^{n}$ by restriction. Some refinements are however possible and we expect to report on this separately.

\section{Appendix A. Equal Closure and continuous maps}

This appendix is concerned with the relation between continuous maps and the property of having equal closure. It supplements Lemma 1.1

Proposition A.1. If $X$ and $Y$ are topological vector spaces (not necessarily Hausdorff) and $0 \in X$ has a countable neighbourhood base, a linear map $\phi: X \mapsto Y$ is continuous if and only if for all subsets $A$ and $B$ of $X$ having equal closure in $X$, the images $\phi(A)$ and $\phi(B)$ again have equal sequential closure in $Y$.

Note that closure and sequential closure coincide in $X$.

Proof. As to the "only if" part, assume that $\phi$ is continuous and $\bar{A}=\bar{B}$. It is sufficient to show that $y \in \overline{\phi(B)}^{s}$ for $y \in \overline{\phi(A)}^{s}$. Let $\left\{a_{n}\right\}_{n=1}^{\infty}$ be a sequence in $A$ such that $\lim _{n \rightarrow \infty} \phi\left(a_{n}\right)=y$. Let $\left\{U_{n}\right\}_{n=1}^{\infty}$ be a neighbourhood base of 0 in $X$. We may assume that $U_{1} \supset U_{2} \supset U_{3} \supset \ldots$. Since $a_{n} \in \bar{A}=\bar{B}$ for all $n$, there exists for all $n$ an element $b_{n} \in B$ such that $a_{n}-b_{n} \in U_{n}$. Then $\lim _{n \rightarrow \infty} \phi\left(b_{n}\right)=y$. To see this, let $V_{0}$ be an arbitrary neighbourhood of 0 in $Y$. Choose a neighbourhood $V_{1}$ of 0 in $Y$ such that $V_{1}+V_{1} \subset V_{0}$. There exists $N_{1}$ such that $y-\phi\left(a_{n}\right) \in V_{1}$ for all $n \geq N_{1}$. By the continuity of $\phi$, there exists $N_{2}$ such that $\phi\left(U_{N_{2}}\right) \subset V_{1}$. Then for all $n \geq \max \left(N_{1}, N_{2}\right)$ we have $y-\phi\left(b_{n}\right)=\left(y-\phi\left(a_{n}\right)\right)+\phi\left(a_{n}-b_{n}\right) \in V_{1}+\phi\left(U_{n}\right) \subset$ $V_{1}+\phi\left(U_{N_{2}}\right) \subset V_{1}+V_{1} \subset V_{0}$.

As to the "if" part, let $\phi$ preserve the property of having equal (sequential) closure. Suppose that $\phi$ is not continuous at 0 . Then there exists an open neighbourhood $V$ of 0 in $Y$ such that $\phi^{-1}(V)$ is not a neighbourhood of 0 in $X$. Using a decreasing neighbourhood base at $0 \in X$ as above, one easily constructs a sequence $\left\{x_{n}\right\}_{n=1}^{\infty} \subset X$ such that $\phi\left(x_{n}\right) \in Y-V$ for all $n$ and such that $x_{n} \rightarrow 0$. Then $\overline{\bigcup_{n=1}^{\infty}\left\{x_{n}\right\} \cup\{0\}}=\overline{\bigcup_{n=1}^{\infty}\left\{x_{n}\right\}}$, so $0=\phi(0) \in{\overline{\bigcup_{n=1}^{\infty}\left\{\phi\left(x_{n}\right)\right\} \cup\{\phi(0)\}}}^{s}=$

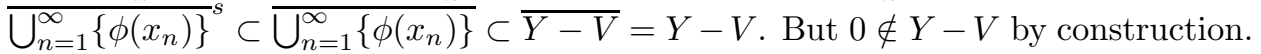
Contradiction.

Theorem A.2. Let $Y$ be a topological vector space. Suppose that $I$ is a nonempty index set and assume that for each $i \in I$ a topological vector space $X_{i}$ and a continuous linear map $\phi_{i}: X_{i} \mapsto Y$ are given. 
Let $\mathcal{C}$ be a choice function, assigning to each $i \in I$ a dense subspace $\mathcal{C}_{i}$ of $X_{i}$, and consider the corresponding algebraic span $L_{\mathcal{C}}=\sum_{i \in I} \phi_{i}\left(\mathcal{C}_{i}\right)$ of the images $\phi_{i}\left(\mathcal{C}_{i}\right)$. Then:

1. $L_{\mathcal{C}}^{\perp}$ is independent of $\mathcal{C}$. In fact, if $\mathcal{C}^{\prime}$ is any other such choice function, then $L_{\mathcal{C}}^{\perp}=\bigcap_{i \in I} \phi_{i}\left(\mathcal{C}_{i}^{\prime}\right)^{\perp}$.

2. If $Y$ is locally convex, then the closure of $L_{\mathcal{C}}$ is independent of $\mathcal{C}$.

3. If I is finite, then the closure of $L_{\mathcal{C}}$ is independent of $\mathcal{C}$.

4. If $I$ is finite and the $X_{i}$ all have a countable neighbourhood base at 0 , then the sequential closure of $L_{\mathcal{C}}$ is independent of $\mathcal{C}$.

Proof. As to (1), invoking Lemma 1.1 yields that

$$
L_{\mathcal{C}}^{\perp}=\bigcap_{i \in I} \phi_{i}\left(\mathcal{C}_{i}\right)^{\perp}=\bigcap_{i \in I}\left\{\overline{\phi_{i}\left(\mathcal{C}_{i}\right)}\right\}^{\perp}=\bigcap_{i \in I}\left\{\overline{\phi_{i}\left(\mathcal{C}_{i}^{\prime}\right)}\right\}^{\perp}=\bigcap_{i \in I} \phi_{i}\left(\mathcal{C}_{i}^{\prime}\right)^{\perp} .
$$

This proves (1) and (2) is then immediate. For the remaining statements assume $I=\{1, \ldots, n\}$. Consider $\widehat{X}=\bigoplus_{i=1}^{n} X_{i}$ in its product topology and define the continuous linear map $\phi: \widehat{X} \mapsto Y$ by $\phi\left(x_{1}, \ldots, x_{n}\right)=\sum_{i=1}^{n} \phi_{i}\left(x_{i}\right)$. Consider the subspace $\widehat{M}_{\mathcal{C}}=\bigoplus_{i=1}^{n} \mathcal{C}_{i}$ of $\widehat{X}$. Since $\widehat{M}_{\mathcal{C}}=\widehat{X}$, Lemma 1.1 implies (3). If the $X_{i}$ are first countable, then so is $\widehat{X}$ and (4) therefore follows from Proposition A.1.

\section{Appendix B. A Denjoy-Carleman type theorem in SEVERAL VARIABles}

In this appendix we prove a theorem on quasi-analytic classes in several variables. If the $M(j, m)$ in the formulation of the theorem are all strictly positive, then with the aid of [20, Theorem 1.8.VII] the result could also be inferred from [15]. In our application of the theorem below in the proof of Theorem 4.9 the $M(j, m)$ are however only known to be non-negative. Therefore we provide an independent proof, which is perhaps somewhat simpler than the proof in [15] where a proof is given by the repeated use of Bang's formulas. We reduce the theorem to the one-dimensional case by induction.

Theorem B.1. For $j=1, \ldots, n$ let $\{M(j, m)\}_{m=0}^{\infty}$ be a sequence of non-negative real numbers. Put $\mu(j, m)=\inf _{k \geq m} M(j, k)^{1 / k}(j=1, \ldots, n ; m=1,2, \ldots)$ and suppose

$$
\sum_{m=1}^{\infty} \frac{1}{\mu(j, m)}=\infty \quad(j=1, \ldots, n) .
$$

Let $R>0$ and suppose $f:(-R, R)^{n} \mapsto \mathbb{C}$ is of class $C^{\infty}$. Assume that there exist non-negative constants $C$ and $r$ such that

$$
\left|\partial^{\alpha} f(x)\right| \leq C r^{|\alpha|} \prod_{j=1}^{n} M\left(j, \alpha_{j}\right)
$$

for all $\alpha \in \mathbb{N}^{n}$ and all $x \in(-R, R)^{n}$. Then, if $\partial^{\alpha} f(0)=0$ for all $\alpha \in \mathbb{N}^{n}, f$ is actually identically zero on $(-R, R)^{n}$.

Proof. By induction. For $n=1$ the result is the Denjoy-Carleman theorem if the $M(1, m)$ are all strictly positive [5, 20]. If $M\left(1, m_{0}\right)=0$ for some $m_{0}$ then $f$ is a polynomial of degree at most $m_{0}$ and the result is obvious.

Assuming the theorem for $n-1$, we define for each multi-index $\left(\alpha_{1}, \ldots, \alpha_{n-1}\right) \in$ $\mathbb{N}^{n-1}$ the function $\phi_{\alpha_{1}, \ldots, \alpha_{n-1}}:(-R, R) \mapsto \mathbb{C}$ by $\phi_{\alpha_{1}, \ldots, \alpha_{n-1}}(t)=\partial_{1}^{\alpha_{1}} \cdots \partial_{n-1}^{\alpha_{n-1}} f(0, \ldots, 0, t)$, 
where we have used the shorthand notation $\partial_{j}=\partial / \partial x_{j}$. Then all derivatives of $\phi_{\alpha_{1}, \ldots, \alpha_{n-1}}$ vanish at $0 \in \mathbb{R}$. Since for $m=0,1, \ldots$ and $t \in(-R, R)$ we have

$$
\left|(d / d t)^{m} \phi_{\alpha_{1}, \ldots, \alpha_{n-1}}(t)\right| \leq\left(C r^{\sum_{j=1}^{n-1} \alpha_{j}} \prod_{j=1}^{n-1} M\left(j, \alpha_{j}\right)\right) r^{m} M(n, m),
$$

the result as established for $n=1$ implies that $\phi_{\alpha_{1}, \ldots, \alpha_{n-1}}$ is identically zero on $(-R, R)$, for arbitrary $\left(\alpha_{1}, \ldots, \alpha_{n-1}\right) \in \mathbb{N}^{n-1}$.

Next, for each $t \in(-R, R)$ define $\psi_{t}:(-R, R)^{n-1} \mapsto \mathbb{C}$ by $\psi_{t}\left(x_{1}, \ldots, x_{n-1}\right)=$ $f\left(x_{1}, \ldots, x_{n-1}, t\right)$. Since $\partial_{1}^{\alpha_{1}} \cdots \partial_{n-1}^{\alpha_{n-1}} \psi_{t}(0, \ldots, 0)=\phi_{\alpha_{1}, \ldots, \alpha_{n-1}}(t)$, the vanishing of all $\phi_{\alpha_{1}, \ldots, \alpha_{n-1}}$ on $(-R, R)$ implies that all derivatives of $\psi_{t}$ vanish at $0 \in \mathbb{R}^{n-1}$, for arbitrary $t \in(-R, R)$. Additionally, we have for all $\left(x_{1}, \ldots, x_{n-1}\right) \in(-R, R)^{n-1}$ and all $t \in(-R, R)$ :

$$
\left|\partial_{1}^{\alpha_{1}} \cdots \partial_{n-1}^{\alpha_{n-1}} \psi_{t}\left(x_{1}, \ldots, x_{n-1}\right)\right| \leq(C M(n, 0)) \cdot r^{\sum_{j=1}^{n-1} \alpha_{j}} \prod_{j=1}^{n-1} M\left(j, \alpha_{j}\right) .
$$

For each $t \in(-R, R)$ the induction hypothesis then implies that $\psi_{t}$ is identically zero on $(-R, R)^{n-1}$, i.e. $f$ vanishes on $(-R, R)^{n}$. This completes the induction step.

\section{REFERENCES}

[1] S.N. Bernstein, On weight functions (Russian), Dokl. Akad. Nauk SSSR 77 (1951), 549-552.

[2] N. Bourbaki, "Topologie Générale I," Hermann, Paris, 1971.

[3] N. Bourbaki, "Topologie Générale II," Hermann, Paris, 1971.

[4] N. Bourbaki, "Topological Vector Spaces, Chapters 1-5," Springer Verlag, Berlin-HeidelbergNew York-London-Paris-Tokyo, 1987.

[5] T. Carleman, "Les fonctions quasi-analytiques," Gauthiers-Villars, Paris, 1926.

[6] L. Carleson, On Berstein's approximation problem, Proc. Amer. Math. Soc. 2 (1951), 953961.

[7] E.M.Chirka, "Complex analytic sets," Kluwer Academic Publishers, Dordrecht-BostonLondon, 1989.

[8] C.F. Dunkl and Y. Xu, "Orthogonal polynomials of several variables," Cambridge University Press, Cambridge-New York-Oakleigh-Madrid-Cape Town, 2001.

[9] B. Fuglede, The multidimensional moment problem, Expo. Math. 1 (1983), 47-65.

[10] T. Hall, Sur l'approximation polynômiale des fonctions continues d'une variable réelle, in "9e Congrès Math. Scand., Helsingfors 1938," Mercator, Helsingfors, 1939.

[11] P.R. Halmos, "Measure Theory," Springer Verlag, New York-Heidelberg-Berlin, 1974.

[12] H. Hamburger, Beiträge zur Konvergenztheorie der Stieltjesschen Kettenbrüche, Math. Z. 4 (1919), 186-222.

[13] J. Horvath, L'approximation polynomiale sur un ensemble non compact, Math. Scand. 2 (1954), 83-90.

[14] J. Horváth, "Topological Vector Spaces and Distributions," Addison-Wesley, Reading-Palo Alto-London-Don Mills, 1966.

[15] V.G. Hryptun, An addition to a theorem of S. Mandelbrojt, Ukrain. Mat. Z. 28 (1976), 841-844. English translation: Ukrainian Math. J. 28 (1976), 655-658.

[16] P. Koosis, "The logarithmic integral I," Cambridge University Press, Cambridge-New YorkNew Rochelle-Melbourne-Sydney, 1988.

[17] A.W. Knapp, "Representation theory of semisimple groups, an overview based on examples," Princeton University Press, Princeton, 1986.

[18] R. Larsen, "Banach algebras, an introduction," Marcel Dekker, New York, 1973.

[19] G.D. Lin, On the moment problems, Statistics \& Probability Letters 35 (1997), 85-90.

[20] S. Mandelbrojt, "Séries adhérentes, régularisation des suites, applications," Gauthiers-Villars, Paris, 1952. 
[21] S. Mandelbrojt, "General theorems of closure," The Rice Institute Pamphlet Special Issue, The Rice Institute, Houston, 1951.

[22] S.N. Mergelyan, Weighted approximation by polynomials, Uspekhi Mat. Nauk 11 (1956), 107-52. English translation: Amer. Math. Soc. Transl. (2) 10 (1958), 59-106.

[23] L. Nachbin, "Elements of approximation theory," Van Nostrand, New York-Chicago-San Francisco, 1967.

[24] L. Nachbin, On the weighted approximation of continuous differentiable functions, Proc. Amer. Math. Soc. 111, (1991), no. 2, 481-485.

[25] L. Nachbin, Sur les algèbres denses de fonctions différentiables sur une variété, C. R. Acad. Sci. Paris 228 (1949), 1549-1551.

[26] A. Ostrowski, Über quasianalytische Funktionen und Bestimmtheit aymptotischer Entwicklungen, Acta Math. 53 (1929), 181-266.

[27] W. Rudin, "Functional Analysis," 2nd edition, McGraw-Hill, Singapore, 1991.

[28] W. Rudin, "Real and Complex Analysis," McGraw-Hill, New York, 1985.

[29] L. Schwartz, "Théorie des distributions," Hermann, Paris, 1978.

[30] F. Treves, "Topological Vector Spaces, Distributions and Kernels," Academic Press, New York-London, 1967.

[31] G. Warner, "Harmonic analysis on semi-simple Lie groups I, II," Springer Verlag, BerlinHeidelberg-New York, 1972.

[32] H. Whitney, On ideals of differentiable functions, Amer. J. Math. 70 (1948), 635-658.

[33] G. Zapata, Bernstein approximation problem for differentiable functions and quasi-analytic weights, Trans. Amer. Math. Soc. 182 (1973), 503-509.

M.F.E. de Jeu, Korteweg-de Vries Institute for Mathematics, University of AmsterDam, Plantage Muidergracht 24, 1018 TV Amsterdam, The Netherlands

E-mail address: mdejeu@science.uva.nl 